Supporting Information

\title{
Synthesis of Different Classes of 6-Membered Gold(I) NHC- Complexes by the Isonitrile Route
}

Thomas Wurm, Florian Mulks, Constantin Böhling, Dominic Riedel, Poorya Zargaran, Matthias Rudolph, Frank Rominger ${ }^{+}$and A. Stephen K. Hashmi*

Organisch-Chemisches Institut, Ruprecht-Karls-Universität Heidelberg, Im Neuenheimer Feld 270, 69120 Heidelberg (Germany)

E-mail: $\underline{\text { hashmi@hashmi.de }}$

$[+]$ Crystallographic investigation

1 General Methods $\quad$ S2

2 Synthetic Part $\quad$ S3

3 NMR Spectra $\quad$ S25

4 Computational Details $\quad$ S56

5 Comments on the X-Ray data $\quad$ S70

$\begin{array}{ll}6 \text { References } & \text { S70 }\end{array}$ 


\section{General Methods}

All reagents and solvents were obtained from Acros, ABCR, Alfa Aesar, Sigma-Aldrich or VWR and were used without further purification unless otherwise noted. Deuterated solvents were purchased from Euriso-Top. Absolute solvents were dried by an MB SPS-800 with the aid of drying columns. Preparation of air- and moisture-sensitive materials was carried out in flames-dried flasks under an atmosphere of nitrogen using Schlenk-techniques. Thin layer chromotography (TLC) was performed using Polygram ${ }^{\circledR}$ precoated plastic sheets SIL $\mathrm{G} / \mathrm{UV}_{254}\left(\mathrm{SiO}_{2}, 0.20 \mathrm{~mm}\right.$ thickness$)$ from Macherey-Nagel. Column chromatography was performed using silica gel (40.0-63.0 nm particel size) from Macherey-Nagel. NMR spectra were recorded on Bruker Avance 500 and Bruker Avance 300 and Bruker ARX-250 spectrometers. Chemical shifts (in ppm) were referenced to residual solvent protons. ${ }^{[1]}$ Signal multiplicity was determined as s (singlet), d (doublet), t (triplet), q (quartet), quin (quintet), sex (sextet), sept (septet), m (multiplet). ${ }^{13} \mathrm{C}$ assignment was achieved via DEPT90 and DEPT135 or HSQC-me spectra. Mass spectra were recorded on a Vacuum Generators ZAB2F, Finnigan MAT TSQ 700 or JEOL JMS-700 spectrometer. GC spectra were recorded on HP Agilent 5890 Series II Plus with a HP 5972 mass analysator. IR spectra (in $\mathrm{cm}^{-1}$ ) were recorded on a Bruker Vector 22 FT-IR. Crystal structure analyses were accomplished on Bruker SmartCCD or Bruker APEX diffractometers. ReactIR ${ }^{\mathrm{TM}}$ studies were performed on a ReactIR $^{\mathrm{TM}}$ Mettler Toledo IC10. 


\section{Synthetic Part}

\section{General Procedure 1 (GP1) for the Synthesis of 2-(Chloropropyl)ammonium Chlorides}

5.00-10.0 mmol of the desired amine and 1.25 equialents of 3-bromo-1-propanol were dissolved in 1-2 $\mathrm{ml}$ of benzonitrile and heated for $12 \mathrm{~h}$ in a sealed tube at $95{ }^{\circ} \mathrm{C}$. After this period, the crude product was washed excessively with petrol ether to remove the benzonitrile/alcohol. The crude product was redissolved in DCM and the solution was washed 3 times with saturated $\mathrm{Na}_{2} \mathrm{CO}_{3}$-solution. The organic layer was dried with $\mathrm{Na}_{2} \mathrm{SO}_{4}$ and the solvent was removed under reduced pressure to yield a viscous oil. This was dissolved in dry dichloromethane $(15.0 \mathrm{ml})$ and $\mathrm{SOCl}_{2}$ (3 equiv.) was added. The mixture was heated at reflux for $12 \mathrm{~h}$. After this period of time, all the volatiles were removed in vacuum and the crude product was recrystallized from EtOH/petrol ether to yield the title compound as off-white crystals.

\section{$N$-(3-chloropropyl)cyclohexanammonium chloride 4a}

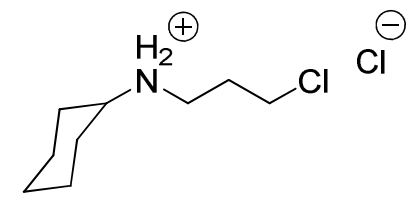

$N$-(3-chloropropyl)cyclohexanammonium chloride 4a was prepared according to GP 1. 623 $\mathrm{mg}(6.28 \mathrm{mmol})$ cyclohexylamine and $916 \mathrm{mg}(6.59 \mathrm{mmol})$ 3-bromo-1-propanol in $0.5 \mathrm{ml}$ benzonitrile were employed. The analytically pure pure alcohol (819 mg, $5.21 \mathrm{mmol}, 83 \%)$ was dissolved $20 \mathrm{ml}$ of dry dichloromethane and $\mathrm{SOCl}_{2}(1.13 \mathrm{ml}, 15.6 \mathrm{mmol})$ was added. After recrystallization the title compound was afforded as colourless crystals; yield: $784 \mathrm{mg}$ (3.70 mmol, 71\%). ${ }^{1} \mathrm{H}$ NMR (300 MHz, $\left.\mathrm{CD}_{2} \mathrm{Cl}_{2}\right): \delta=1.31-1.38(\mathrm{~m}, 3 \mathrm{H}), 1.50-1.71(\mathrm{~m}, 3 \mathrm{H})$, 1.78-1.93 (m, 2H), 2.17-2.28 (m, 2H), 2.39 (t, $J=6.3 \mathrm{~Hz}, 1 \mathrm{H}), 2.45$ (t, $J=6.3 \mathrm{~Hz}, 1 \mathrm{H}), 2.89-$ $3.03(\mathrm{~m}, 1 \mathrm{H}), 3.05-3.14(\mathrm{~m}, 2 \mathrm{H}), 3.72(\mathrm{t}, J=6.9 \mathrm{~Hz}, 2 \mathrm{H}), 9.57(\mathrm{bs}, 2 \mathrm{H}) ;{ }^{13} \mathrm{C} \mathrm{NMR}(75 \mathrm{MHz}$, $\left.\mathrm{CD}_{2} \mathrm{Cl}_{2}\right): \delta=24.95(\mathrm{t}, 2 \mathrm{C}), 25.37(\mathrm{t}), 29.19(\mathrm{t}), 29.52(\mathrm{t}, 2 \mathrm{C}), 42.43(\mathrm{t}), 42.63(\mathrm{t}), 57.93(\mathrm{~d}) ; \mathrm{IR}$ $(\mathrm{KBr}): v=3430,2939,2859,2829,2740,2495,2427,1630,1577,1454,1383,1310,1290$, 1026, 995, 897, 784, $659 \mathrm{~cm}^{-1}$; HRMS (ESI+): found: $\mathrm{m} / z=387.2096$, calcd. for $\mathrm{C}_{18} \mathrm{H}_{38} \mathrm{Cl}_{3} \mathrm{~N}_{2}$ $[2 \mathrm{M}-\mathrm{Cl}]^{+}: 387.2095$. 


\section{$N$-(3-chloropropyl)cyclooctanammonium chloride $4 b$}<smiles>ClCCCNC1CCCCCCC1</smiles>

$\mathrm{N}$-(3-chloropropyl)cyclooctanammonium chloride $\mathbf{4 b}$ was prepared according to GP 1. $1.54 \mathrm{~g}$ (6.84 mmol) cyclooctylamine and $1.00 \mathrm{~g}$ (7.19 mmol) 3-bromo-1-propanol in $0.5 \mathrm{ml}$ benzonitrile were employed. The analytically pure pure alcohol (1.10 g, $5.95 \mathrm{mmol}, 87.0 \%)$ was dissolved $20 \mathrm{ml}$ of dry dichloromethane and $\mathrm{SOCl}_{2}(1.29 \mathrm{ml}, 17.8 \mathrm{mmol})$ was added. After recrystallization the title compound was afforded as colourless crystals; yield: $1.16 \mathrm{~g}$ (4.82 mmol, 81\%). ${ }^{1} \mathrm{H}$ NMR (300 MHz, $\left.\mathrm{CD}_{2} \mathrm{Cl}_{2}\right): \delta=1.33-1.68(\mathrm{~m}, 8 \mathrm{H}), 1.74-1.94(\mathrm{~m}, 4 \mathrm{H})$, 2.43 (quin, $\mathrm{J}=6.7 \mathrm{~Hz}, 2 \mathrm{H}), 3.03-3.13(\mathrm{~m}, 2 \mathrm{H}), 3.05-3.27(\mathrm{~m}, 3 \mathrm{H}), 3.74(\mathrm{t}, \mathrm{J}=6.7 \mathrm{~Hz}, 2 \mathrm{H})$, 9.48 (bs, 2H); ${ }^{13} \mathrm{C}$ NMR (75 MHz, $\left.\mathrm{CD}_{2} \mathrm{Cl}_{2}\right): \delta=24.43(\mathrm{t}, 2 \mathrm{C}), 26.16(\mathrm{t}), 26.74(\mathrm{t}, 2 \mathrm{C}), 29.64$ $(\mathrm{t}), 31.18(\mathrm{t}, 2 \mathrm{C}), 42.68(\mathrm{t}), 42.86(\mathrm{t}), 59.36(\mathrm{~d}) ; \mathrm{IR}(\mathrm{KBr}): v=3422,2932,2855,2809,2481$, 2412, 1586, 1451, 1362, 1317, 1047, 739, 656, $545 \mathrm{~cm}^{-1}$; HR-MS (ESI+): found: $\mathrm{m} / \mathrm{z}=$ 204.1513, calcd. for $\mathrm{C}_{11} \mathrm{H}_{23} \mathrm{ClN}[\mathrm{M}-\mathrm{Cl}]+:$ : 04.1514 .

\section{$N$-(3-chloropropyl)cyclododecanammonium chloride 4c}

was prepared according to the literature procedure. ${ }^{[1]}$

\section{$N$-(3-chloropropyl)cyclopentadecanammonium chloride 4d}

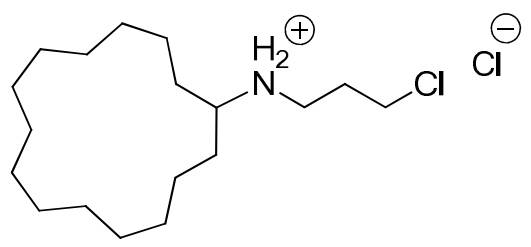

$\mathrm{N}$-(3-chloropropyl)cyclopentadecanammonium chloride was prepared according to GP $\mathbf{1}$. $1.54 \mathrm{~g}$ (6.84 mmol) cyclopentadecylamine and $1.00 \mathrm{~g}$ (7.19 mmol) 3-bromo-1-propanol in 0.5 $\mathrm{ml}$ benzonitrile were employed. The analytically pure pure alcohol (1.66 g, $5.85 \mathrm{mmol}, 86 \%)$ was dissolved $25 \mathrm{ml}$ of dry dichloromethane and $\mathrm{SOCl}_{2}(1.31 \mathrm{ml}, 17.9 \mathrm{mmol})$ was added. After recrystallization the title compound was afforded as colourless crystals; yield: $1.76 \mathrm{~g}$ 
(5.21 mmol, 89\%). ${ }^{1} \mathrm{H}$ NMR (300 MHz, $\left.\mathrm{CD}_{2} \mathrm{Cl}_{2}\right): \delta=1.24-1.53\left(\mathrm{~m}, 24 \mathrm{H}, \mathrm{CH}_{2}\right), 1.70-1.95(\mathrm{~m}$, 4H), $2.44(\mathrm{t}, J=6.3 \mathrm{~Hz}, 1 \mathrm{H}), 2.48(\mathrm{t}, J=6.3 \mathrm{~Hz}, 1 \mathrm{H}), 2.96-3.16(\mathrm{~m}, 3 \mathrm{H}), 3.73(\mathrm{t}, J=6.3 \mathrm{~Hz}$, 2H), 9.53 (bs, 2H, NH2); ${ }^{13} \mathrm{C}$ NMR (75 MHz, $\mathrm{CD}_{2} \mathrm{Cl}_{2}$ ): $\delta=24.23(\mathrm{t}, 2 \mathrm{C}), 26.72(\mathrm{t}, 2 \mathrm{C}), 26.90$ $(\mathrm{t}, 2 \mathrm{C}), 27.08(\mathrm{t}, 2 \mathrm{C}), 27.18(\mathrm{t}, 2 \mathrm{C}), 27.58(\mathrm{t}, 2 \mathrm{C}), 29.09(\mathrm{t}), 30.16(\mathrm{t}, 2 \mathrm{C}), 42.66(\mathrm{t}), 42.86(\mathrm{t})$, 58.58 (d); IR (KBr): $v=3432,2929,2857,2807,2493,2412,1699,1684,1635,1586,1559$, 1541, 1521, 1507, 1459, 1351, 1320, 1020, 736, $653 \mathrm{~cm}^{-1}$; HR-MS (ESI+): found: $\mathrm{m} / z=$ 302.2609, calcd. for $\mathrm{C}_{18} \mathrm{H}_{37} \mathrm{ClN}$ [M-Cl]+: 302.2615 .

\section{2-(2-chloroethyl)piperidin-1-ium chloride 4e}

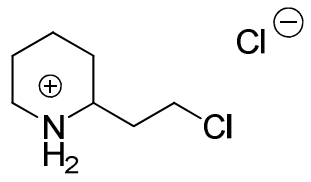

2-(2-chloroethyl)piperidin-1-ium chloride was prepared according to GP 1. $1.00 \mathrm{~g}$ (7.74 mmol) commercially available 2-(2-hydroxyethyl)piperidine was dissolved $35 \mathrm{ml}$ of dry dichloromethane and $\mathrm{SOCl}_{2}(1.68 \mathrm{ml}, 23.2 \mathrm{mmol})$ was added. After recrystallization the title compound was afforded as colourless crystals; yield: $1.27 \mathrm{~g}$ (6.89 mmol, 89\%). ${ }^{1} \mathrm{H}$ NMR (300 $\left.\mathrm{MHz}, \mathrm{CD}_{2} \mathrm{Cl}_{2}\right): \delta=1.41-1.56(\mathrm{~m}, 1 \mathrm{H}), 1.62-1.78(\mathrm{~m}, 1 \mathrm{H}), 1.79-1.98(\mathrm{~m}, 3 \mathrm{H}), 2.09-2.22(\mathrm{~m}$, $1 \mathrm{H}), 2.45-2.64(\mathrm{~m}, 1 \mathrm{H}), 2.76-2.89(\mathrm{~m}, 1 \mathrm{H}), 3.14-3.28(\mathrm{~m}, 1 \mathrm{H}), 3.36-3.46(\mathrm{~m}, 1 \mathrm{H}), 3.66-3.75$ $(\mathrm{m}, 1 \mathrm{H}), 3.85-3.93(\mathrm{~m}, 1 \mathrm{H}), 9.53(\mathrm{bs}, 1 \mathrm{H}), 9.87$ (bs, $1 \mathrm{H}) ;{ }^{13} \mathrm{C} \mathrm{NMR}\left(75 \mathrm{MHz}, \mathrm{CD}_{2} \mathrm{Cl}_{2}\right): \delta=$ $22.49(\mathrm{t}), 22.75(\mathrm{t}), 28.41(\mathrm{t}), 36.29(\mathrm{t}), 41.00(\mathrm{t}), 45.08(\mathrm{~d}), 54.92(\mathrm{t}) ; \mathrm{IR}(\mathrm{KBr}): v=3444$, 2949, 2801, 2723, 2599, 2565, 2510, 2432, 2394, 1578, 1455, 1432, 1328, 1305, 1285, 1032, $1018,949,656,553 \mathrm{~cm}^{-1}$; HR-MS (EI+): found: $m / z=147.0809$, calcd. for $\mathrm{C}_{7} \mathrm{H}_{14} \mathrm{ClN}$ [M$\mathrm{Cl}]^{+}: 147.0815$.

\section{1-Chloro-3-(cyclohexylamino)propan-2-ol 4f $\mathrm{f}^{[2]}$}<smiles>OC(CCl)CNC1CCCCC1</smiles>

Cyclohexylamine $1.4 \mathrm{ml}(12.2 \mathrm{mmol}, 1.20 \mathrm{~g})$ was dissolved in pentane $(6 \mathrm{~mL})$ and epichlorhydrine $(1.0 \mathrm{~mL}, 1.18 \mathrm{~g}, 12.8 \mathrm{mmol}, 1.05$ eq.) was added. The reaction mixture was 
stirred for 48 hours at rt. After that the volatiles were decanted and the precipitate was washed with cold pentane $(3 \times 10 \mathrm{~mL})$. After removing of all volatiles in vacuo $972 \mathrm{mg}(5.07 \mathrm{mmol}$, $40 \%)$ of the product $\mathbf{4 f}$ was achieved as colourless solid; ${ }^{1} \mathrm{H}$ NMR $\left(300 \mathrm{MHz}, \mathrm{CDCl}_{3}\right): \delta=$ 0.97-1.30 (m; 5H), 1.55-1.61 (m; 1H), 1.67-1.74 (m;2H), 1.85-1.90 (m;2H), $2.40(\mathrm{tt}, \mathrm{J}=$ 10.4, 3.8 Hz; 1H), 2.65 (dd, J = 12.3, 7.9 Hz; 1H), 2.74 (bs; 2H), 2.85 (dd, J = 12.2, 4.0 Hz; 1 $\mathrm{H}), 3.52(\mathrm{~d}, \mathrm{~J}=5.4 \mathrm{~Hz} ; 2 \mathrm{H}), 3.81(\mathrm{ddt}, \mathrm{J}=7.9,5.5,3.9 \mathrm{HZ} ; 1 \mathrm{H}),{ }^{13} \mathrm{C}$ NMR $(75.6 \mathrm{MHz}$, $\left.\mathrm{CDCl}_{3}\right): \delta=25.16(\mathrm{t}, 2 \mathrm{C}), 26.17(\mathrm{t}), 33.68(\mathrm{t}), 33.91(\mathrm{t}), 47.52(\mathrm{t}), 49.26(\mathrm{t}), 57.03(\mathrm{~d}), 69.64$ (d). IR (KBr): $v=3277,3097,2956,2931,2858,1462,1448,1437,1154,1123,1091,1075$, 1050, 974, 933, 917, 897, 873, 843, 740; HR-MS (EI+): $\mathrm{C}_{9} \mathrm{H}_{17} \mathrm{ClNO}[\mathrm{M}-\mathrm{H}]^{+}$, calc. 190.0993, found 190.0999; $\mathrm{EA}\left(\mathrm{C}_{9} \mathrm{H}_{18} \mathrm{ClNO}\right)$ : calc. C 56.39, H 9.46, N 7.31, found C 56.33, H 9.53, N 7.36.

\section{General Procedure 2 (GP 2) for the Synthesis of Six-Membered saturated NHC Gold(I) Complexes}

1.25 equivalents of an 2-(chloropropyl)alkylammonium chloride and $\mathrm{NEt}_{3}$ (10.0 equivalents) were added to a $0.5 \mathrm{M}$ solution of an isocyanogold(I) complex in dry dichloromethane under an atmosphere of nitrogen. The mixture was stirred for $12 \mathrm{~h}$ at RT. After this period, the solvent was removed under reduced pressure and the crude product was dissolved in DCM and washed with saturated $\mathrm{NH}_{4} \mathrm{Cl}$ solution. The organic layer was dried over $\mathrm{Na}_{2} \mathrm{SO}_{4}$, filtered and all volatiles were removed under reduced pressure. The crude product was purified through a short plug of silica (dichloromethane as eluent) to afford the title compund as colourless crystaline solid.

6a

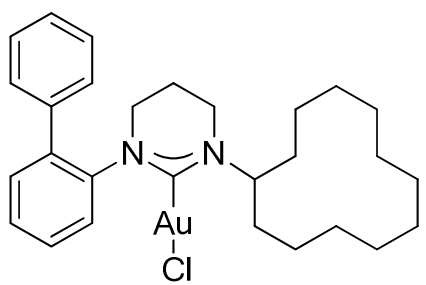

6a was prepared according to GP 2. $100 \mathrm{mg}$ (243 $\mu \mathrm{mol})$ 2'-biphenylisocyanogold(I)chloride, $107 \mathrm{mg}(364 \mu \mathrm{mol}) 4 \mathrm{c}^{[1]}$ and $0.5 \mathrm{ml} \mathrm{NEt}_{3}$ in $5 \mathrm{ml}$ of dry dichloromethane were employed. Purification by column chromatography afforded a colourless crystaline solid; yield: $137 \mathrm{mg}$ ( 
$216 \mu \mathrm{mol}, 89 \%) ;{ }^{1} \mathrm{H}$ NMR (300 MHz, $\left.\mathrm{CD}_{2} \mathrm{Cl}_{2}\right): \delta=1.18-1.63(\mathrm{~m}, 19 \mathrm{H}), 1.64-1.88(\mathrm{~m}, 5 \mathrm{H})$, 2.91-2.99 (m, 2H), 3.05 (ddd, $J=12.9 \mathrm{~Hz}, J=7.8 \mathrm{~Hz}, J=4.7 \mathrm{~Hz}, 1 \mathrm{H}), 3.22-3.33$ (m, 1H), 5.02-5.14 (m, 1H), 7.32-7.53 (m, 9H); ${ }^{13} \mathrm{C}$ NMR (75 MHz, $\left.\mathrm{CD}_{2} \mathrm{Cl}_{2}\right): \delta=20.65(\mathrm{t}), 22.51(\mathrm{t})$, $22.52(\mathrm{t}), 22.68(\mathrm{t}), 23.30(\mathrm{t}), 23.32(\mathrm{t}), 24.06(\mathrm{t}), 24.27(\mathrm{t}), 25.14(\mathrm{t}), 25.33(\mathrm{t}), 27.51(\mathrm{t}), 29.36$ (t), $39.35(\mathrm{t}), 48.83(\mathrm{t}), 63.97$ (d), 127.96 (d), 128.63 (d, 2C), 128.92 (d), 129.06 (d), 129.55 (d, 2C), 129.86 (d), 131.47 (d), 139.34 (s), 139.69 (s), 145.98 (s), 190.77 (s); IR (KBr): v = 3432, 3057, 2860, 1630, 1523, 1479, 1445, 1414, 1354, 1310, 1265, 1207, 1169, 1074, 1022, 806, 763, 741, $702 \mathrm{~cm}-1$; HR-MS (FAB+): found: $m / z=634.2410$, calcd. for $\mathrm{C}_{28} \mathrm{H}_{38} \mathrm{AuClN}_{2}$ $[\mathrm{M}]^{+}: 634.2389$.

6b

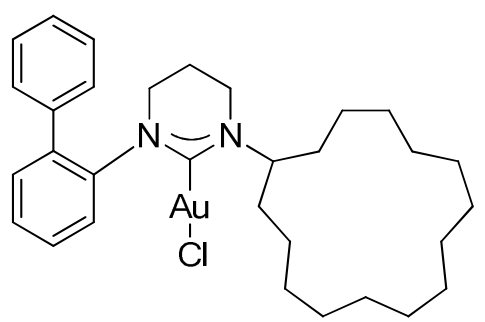

6b was prepared according to GP 2. $100 \mathrm{mg}$ (243 $\mu \mathrm{mol})$ 2'Biphenylisocyanogold(I) chloride, $123 \mathrm{mg}(364 \mu \mathrm{mol}) \mathbf{4 d}$ and1.00 $\mathrm{ml} \mathrm{NEt}_{3}$ in $5 \mathrm{ml}$ of dry dichloromethane were employed. Purification by column chromatography afforded a colourless crystaline solid; yield: $153 \mathrm{mg}$ (226 $\mu \mathrm{mol}, 93 \%) ;{ }^{1} \mathrm{H}$ NMR (300 MHz, $\left.\mathrm{CD}_{2} \mathrm{Cl}_{2}\right): \delta=1.11-1.46(\mathrm{~m}, 24 \mathrm{H}), 1.47-1.80(\mathrm{~m}, 6 \mathrm{H})$, 2.79-2.98 (m, 3H), 3.20-3.27 (m, 1H), 4.92-5.00 (m, 1H), 7.26-7.39 (m, 7H), 7.41-7.46 (m, $2 \mathrm{H}) ;{ }^{13} \mathrm{C}$ NMR $\left(75 \mathrm{MHz}, \mathrm{CD}_{2} \mathrm{Cl}_{2}\right): \delta=20.51(\mathrm{t}), 24.61(\mathrm{t}), 25.01(\mathrm{t}), 27.18(\mathrm{t}, 2 \mathrm{C}), 27.26(\mathrm{t})$, $27.36(\mathrm{t}), 27,39(\mathrm{t}), 27,41(\mathrm{t}), 27,45(\mathrm{t}, 3 \mathrm{C}), 27.57(\mathrm{t}), 30.23(\mathrm{t}), 31.26(\mathrm{t}), 38.86(\mathrm{t}), 48.20(\mathrm{t})$, 66.76 (d), 127.98 (d), 128.65 (d, 2C), 128.91 (d), 129.04 (d), 129.55 (d, 2C), 129.96 (d), 131.46 (d), 139.31 (s), 139.60 (s), 146.62 (s), 190.77 (s); IR (KBr): v = 3429, 3057, 2928, 2854, 1629, 1523, 1480, 1452, 1414, 1355, 1310, 1271, 1207, 1158, 1073, 1046, 761, 741, 703, $605 \mathrm{~cm}^{-1}$; HR-MS (FAB+): found: $m / z=676.2813$, calcd. for $\mathrm{C}_{31} \mathrm{H}_{44} \mathrm{AuClN}_{2}[\mathrm{M}]^{+}$: 676.2859, $m / z=641.3181$ calcd. for $\mathrm{C}_{31} \mathrm{H}_{44} \mathrm{AuN}_{2}[\mathrm{M}-\mathrm{Cl}]^{+}: 641.3170$. 


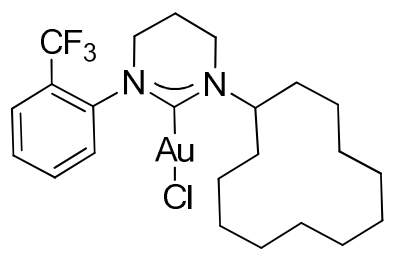

6c was prepared according to GP 2. $100 \quad \mathrm{mg} \quad(249 \quad \mu$ mol) 2 (Trifluormethyl)phenylisocyanogold(I) chloride, $107 \quad \mathrm{mg} \quad(469 \mu \mathrm{mol}) \quad \mathrm{N}-(2-$ chloropropyl)cyclododecanammonium chloride $4 \mathrm{c}^{[1]}$ and $0.5 \mathrm{ml} \mathrm{NEt}_{3}$ in $5 \mathrm{ml}$ of dry dichloromethane were employed. Purification by column chromatography afforded a colourless crystaline solid; yield: $126 \mathrm{mg}(380 \mu \mathrm{mol}, 81 \%) ;{ }^{1} \mathrm{H} \mathrm{NMR}\left(300 \mathrm{MHz}, \mathrm{CD}_{2} \mathrm{Cl}_{2}\right)$ : $\delta=$ $1.21-1.44(\mathrm{~m}, 13 \mathrm{H}), 1.44-1.61(\mathrm{~m}, 5 \mathrm{H}), 1.70-1.86(\mathrm{~m}, 4 \mathrm{H}), 2.02-2.23(\mathrm{~m}, 2 \mathrm{H}), 3.18-3.34(\mathrm{~m}$, 2H), 3.41-3.49 (m, 1H), 3.51-3.60 (m, 1H), 5.09 (quin, $J=6.7 \mathrm{~Hz}, 1 \mathrm{H}), 7.43(\mathrm{~d}, J=7.8 \mathrm{~Hz}$, $1 \mathrm{H}), 7.58(\mathrm{t}, J=7.8 \mathrm{~Hz}, 1 \mathrm{H}), 7.68-7.77(\mathrm{~m}, 2 \mathrm{H}) ;{ }^{13} \mathrm{C} \mathrm{NMR}\left(75 \mathrm{MHz}, \mathrm{CD}_{2} \mathrm{Cl}_{2}\right): \delta=19.78(\mathrm{t})$, $21.32(\mathrm{t}, 2 \mathrm{C}), 21.48(\mathrm{t}), 22.07(\mathrm{t}), 22.16(\mathrm{t}), 22.88(\mathrm{t}), 23.06(\mathrm{t}), 23.91(\mathrm{t}), 24.09(\mathrm{t}), 26.47(\mathrm{t})$, $28.08(\mathrm{t}), 38.47(\mathrm{t}), 48.36(\mathrm{t}), 63.20(\mathrm{~d}), 122.91\left(\mathrm{q}, \mathrm{J}_{\mathrm{CF}}=273 \mathrm{~Hz}\right), 126.88\left(\mathrm{q}, \mathrm{J}_{\mathrm{CF}}=30 \mathrm{~Hz}\right)$, $126.98\left(\mathrm{dq}, \mathrm{J}_{\mathrm{CF}}=5.3 \mathrm{~Hz}\right), 128.17$ (d), 130.74 (d), 133.05 (d), 144.59 (q, J $\left.\mathrm{J}_{\mathrm{CF}}=1.6 \mathrm{~Hz}\right), 189.82$ $\left(\mathrm{q}, \mathrm{J}_{\mathrm{CF}}=2.3 \mathrm{~Hz}\right), \mathrm{IR}(\mathrm{KBr}): v=3433,2931,2862,1605,1527,1497,1470,1455,1415,1355$, $1314,1261,1209,1173,1129,1173,1129,1075,1054,1035,771,599 \mathrm{~cm}^{-1}$; HR-MS $\left(\mathrm{FAB}+\right.$ ): found: $m / z=626.1920$, calcd. for $\mathrm{C}_{23} \mathrm{H}_{33} \mathrm{AuClF}_{3} \mathrm{~N}_{2}[\mathrm{M}]^{+}: 626.1950, m / z=591.2246$, calcd. for $\mathrm{C}_{23} \mathrm{H}_{33} \mathrm{AuF}_{3} \mathrm{~N}_{2}[\mathrm{M}-\mathrm{Cl}]^{+}: 591.2261$.

6d

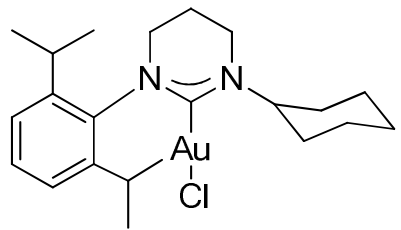

6d was prepared according to GP 2. $100 \quad \mathrm{mg} \quad(238 \mu \mathrm{mol})$ 2,6diisopropylphenylisocyanogold(I) chloride, $63.1 \mathrm{mg}(298 \mu \mathrm{mol}) \mathbf{4 a}$ and $0.5 \mathrm{ml} \mathrm{NEt}_{3}$ in $5 \mathrm{ml}$ of dry dichloromethanewere employed. Purification by column chromatography afforded a colourless crystalline solid; yield: $121 \mathrm{mg}$ ( $217 \mu \mathrm{mol}, 91 \%) ;{ }^{1} \mathrm{H} \mathrm{NMR}\left(300 \mathrm{MHz}, \mathrm{CD}_{2} \mathrm{Cl}_{2}\right)$ : $\delta$ $=1.22(\mathrm{~d}, J=6.9 \mathrm{~Hz}, 6 \mathrm{H}), 1.37(\mathrm{~d}, J=6.9 \mathrm{~Hz}, 6 \mathrm{H}), 1.42-1.47(\mathrm{~m}, 2 \mathrm{H}), 1.48-1.74(\mathrm{~m}, 4 \mathrm{H})$, 1.83-1.94 (m, 4H), 2.09-2.16 (m, 2H), 2.90 (sept, $J=6.9$ Hz, 2H), 3.28-3.35 (m, 4H), 4.75 (tt, $J=12.0 \mathrm{~Hz}, J=3.6 \mathrm{~Hz}, 1 \mathrm{H}), 7.22(\mathrm{~d}, J=7.9 \mathrm{~Hz}, 2 \mathrm{H}), 7.40(\mathrm{t}, J=7.9 \mathrm{~Hz}, 1 \mathrm{H}) ;{ }^{13} \mathrm{C} \mathrm{NMR}(75$ 
$\left.\mathrm{MHz}, \mathrm{CD}_{2} \mathrm{Cl}_{2}\right): \delta=20.87(\mathrm{t}), 24.56(\mathrm{q}, 2 \mathrm{C}), 24.81(\mathrm{q}, 2 \mathrm{C}), 25.68(\mathrm{t}), 25.89(\mathrm{t}, 2 \mathrm{C}), 28.68(\mathrm{~d}$, 2C), $30.91(\mathrm{t}, 2 \mathrm{C}), 39.61(\mathrm{t}), 48.98$ (t), 63.40 (d), 124.91 (d, 2C), 129.44 (d), 142.60 (s), 146.29 (s, 2C), 190.28 (s); IR (KBr): $v=3443,2961,2924,2864,1621,1590,1525,1455$, 1414, 1383, 1361, 1307, 1256, 1214, 1152, 1105, 1071, 1053, 805, $760 \mathrm{~cm}^{-1}$; HR-MS $\left(\mathrm{FAB}+\right.$ ): found: $m / z=558.2087$, calcd. for $\mathrm{C}_{22} \mathrm{H}_{34} \mathrm{AuClN}_{2}[\mathrm{M}]^{+}:$558.2076.

6e

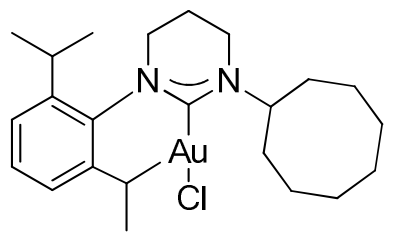

6e was prepared according to GP 2. $100 \quad \mathrm{mg} \quad(238 \mu \mathrm{mol})$ 2,6diisopropylphenylisocyanogold(I) chloride, $115 \mathrm{mg}(477 \mu \mathrm{mol}) 4 \mathbf{b}$ and $0.5 \mathrm{ml} \mathrm{NEt}_{3}$ in $5 \mathrm{ml}$ of dry dichloromethane were employed. Purification by column chromatography afforded a colourless crystalline solid; yield: $116 \mathrm{mg}(197 \mu \mathrm{mol}, 83 \%) ;{ }^{1} \mathrm{H} \mathrm{NMR}\left(300 \mathrm{MHz}, \mathrm{CD}_{2} \mathrm{Cl}_{2}\right): \delta$ $=1.23(\mathrm{~d}, J=6.9 \mathrm{~Hz}, 6 \mathrm{H}), 1.37(\mathrm{~d}, J=6.9 \mathrm{~Hz}, 6 \mathrm{H}), 1.49-1.94(\mathrm{~m}, 14 \mathrm{H}), 2.07-2.15(\mathrm{~m}, 2 \mathrm{H})$, 2.90 (sept, $J=6.9 \mathrm{~Hz}, 2 \mathrm{H}), 3.27-3.34(\mathrm{~m}, 4 \mathrm{H}), 5.04-5.17(\mathrm{~m}, 1 \mathrm{H}), 7.22(\mathrm{~d}, J=7.9 \mathrm{~Hz}, 2 \mathrm{H})$, $7.40(\mathrm{t}, J=7.9 \mathrm{~Hz}, 1 \mathrm{H}) ;{ }^{13} \mathrm{C} \mathrm{NMR}\left(75 \mathrm{MHz}, \mathrm{CD}_{2} \mathrm{Cl}_{2}\right): \delta=21.01(\mathrm{t}), 24.64$ (q, 2C), 24.84 (q, 2C), $25.26(t, 2 C), 26.59(t), 26.75(t, 2 C), 28.67(d, 2 C), 32.25(t, 2 C), 39.80(t), 48.94(t)$, 69.06 (d), 124.89 (d, 2C), 129.41 (d), 142.52 (s), 146.22 (s, 2C), 189.45 (s); IR (KBr): v = 3444, 2961, 2924, 2865, 1621, 1590, 1525, 1454, 1414, 1383, 1361, 1307, 1256, 1214, 1151, 1105, 1068, 1055, 805, $760 \mathrm{~cm}^{-1}$; HR-MS (FAB+): found: $m / z=586.2354$, calcd. for $\mathrm{C}_{24} \mathrm{H}_{38} \mathrm{AuClN}_{2}[\mathrm{M}]^{+}:$586.2389; found: $m / z=551.2696$, calcd. for $\mathrm{C}_{24} \mathrm{H}_{38} \mathrm{AuN}_{2}[\mathrm{M}-\mathrm{Cl}]^{+}$: 551.2701 .

6f

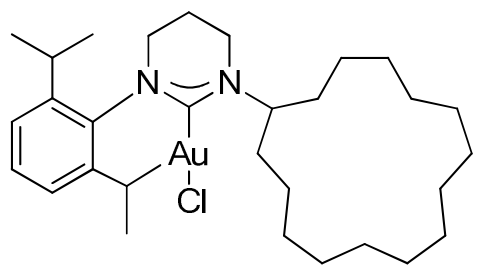

6f was prepared according to GP 2. $143 \mathrm{mg}(341 \mu \mathrm{mol})$ 2,6-diisopropylphenylisocyanogold(I) chloride, $230 \mathrm{mg}(681 \mu \mathrm{mol}) \mathbf{4 d}$ and $1.00 \mathrm{ml} \mathrm{NEt} 3$ in $7 \mathrm{ml}$ of dry dichloromethane were employed. Purification by column chromatography afforded a colourless crystaline solid; 
yield: $189 \mathrm{mg}(276 \mu \mathrm{mol}, 81 \%) ;{ }^{1} \mathrm{H}$ NMR (300 MHz, $\left.\mathrm{CD}_{2} \mathrm{Cl}_{2}\right): \delta=1.19-1.53(\mathrm{~m}, 22 \mathrm{H}), 1.24$ $(\mathrm{d}, J=6.9 \mathrm{~Hz}, 6 \mathrm{H}), 1.37(\mathrm{~d}, J=6.9 \mathrm{~Hz}, 6 \mathrm{H}), 1.59-1.69(\mathrm{~m}, 4 \mathrm{H}), 1.70-1.78(\mathrm{~m}, 2 \mathrm{H}), 2.14$ (pent, $\mathrm{J}=5.8 \mathrm{~Hz}, 2 \mathrm{H}), 2.90$ (sept, $J=6.9 \mathrm{~Hz}, 2 \mathrm{H}), 3.25$ (t, $J=5.8 \mathrm{~Hz}, 2 \mathrm{H}), 3.32$ (t, J = 5.8 Hz, $2 \mathrm{H}), 4.95-5.02(\mathrm{~m}, 1 \mathrm{H}), 7.22(\mathrm{~d}, J=7.8 \mathrm{~Hz}, 2 \mathrm{H}), 7.40(\mathrm{t}, J=7.8 \mathrm{~Hz}, 1 \mathrm{H}) ;{ }^{13} \mathrm{C} \mathrm{NMR}(75 \mathrm{MHz}$, $\mathrm{CD}_{2} \mathrm{Cl}_{2}$ ): $\delta=20.83(\mathrm{t}), 24.59$ (q, 2C), 24.76 (q, 2C), 24.87 (t, 2C), $27.11(\mathrm{t}, 2 \mathrm{C}), 27.30(\mathrm{t}, 2 \mathrm{C})$, $27.36(t, 2 \mathrm{C}), 27.44(\mathrm{t}, 2 \mathrm{C}), 27.56(\mathrm{t}, 2 \mathrm{C}), 28.73(\mathrm{~d}, 2 \mathrm{C}), 30.66(\mathrm{t}, 2 \mathrm{C}), 38.86(\mathrm{t}), 49.11(\mathrm{t})$, 66.26 (d), 124.88 (d, 2C), 129.42 (d), 142.44 (s), 146.28 (s, 2C), 191.08 (s); IR (KBr): v = 3444, 3065, 2960, 2927, 2856, 1634, 1591, 1524, 1457, 1414, 1384, 1359, 1310, 1266, 1208, 1177, 1104, 1056, 804, $758 \mathrm{~cm}^{-1}$; HR-MS (FAB+): found: $m / z=684.3509$, calcd. for $\mathrm{C}_{31} \mathrm{H}_{52} \mathrm{AuClN}_{2}[\mathrm{M}]+:$ 684.3485, $m / z=649.3802$ calcd. for $\mathrm{C}_{31} \mathrm{H}_{52} \mathrm{AuN}_{2}[\mathrm{M}-\mathrm{Cl}]^{+}: 649.3796$.

\section{$6 \mathrm{~g}$}

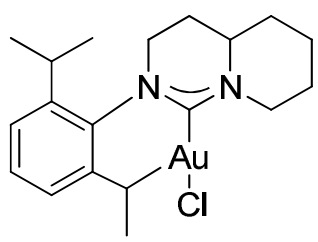

6g was prepared according to GP 2. $100 \quad \mathrm{mg} \quad(191 \mu \mathrm{mol})$ 2,6diisopropylphenylisocyanogold(I) chloride, $43.8 \mathrm{mg}(238 \mu \mathrm{mol}) 4 \mathrm{e}$ and $1.00 \mathrm{ml} \mathrm{NEt}_{3}$ in $5 \mathrm{ml}$ of dry dichloromethane were employed. Purification by column chromatography afforded a colourless crystaline solid; yield: $81.7 \mathrm{mg}(154 \mu \mathrm{mol}, 82 \%) ;{ }^{1} \mathrm{H} \mathrm{NMR}\left(300 \mathrm{MHz}, \mathrm{CD}_{2} \mathrm{Cl}_{2}\right)$ : $\delta$ $=1.22(\mathrm{~d}, J=6.9 \mathrm{~Hz}, 3 \mathrm{H}), 1.24(\mathrm{~d}, J=6.9 \mathrm{~Hz}, 3 \mathrm{H}), 1.35$ (d, $J=6.9 \mathrm{~Hz}, 3 \mathrm{H}), 1.39$ (d, $J=6.9$ $\mathrm{Hz}, 3 \mathrm{H}), 1.46-1.75$ (m, 3H), 1.79-1.89 (m, 2H), 1.94-2.05 (m, 2H), 2.23-2.30 (m, 1H), 2.91 (sept, $J=6.9 \mathrm{~Hz}, 2 \mathrm{H}), 3.26-3.37$ (m, 3H), 3.42-3.53 (m, 1H), 4.96-5.05 (m, 1H), 7.23 (d, $J=$ $7.7 \mathrm{~Hz}, 2 \mathrm{H}), 7.40$ (t, $J=7.7 \mathrm{~Hz}, 1 \mathrm{H}) ;{ }^{13} \mathrm{C} \mathrm{NMR}\left(75 \mathrm{MHz}, \mathrm{CD}_{2} \mathrm{Cl}_{2}\right): \delta=24.45$ (q), 24.49 (q), $24.71(\mathrm{q}), 24.84(\mathrm{t}), 24.98$ (q), 27.16 (t), $27.42(\mathrm{t}), 28.76$ (d), $28.92(\mathrm{t}), 33.86(\mathrm{t}), 45.58(\mathrm{t})$, 53.96 (d), 58.59 (t), 124.89 (d), 124.91 (d), 129.51 (d), 142.54 (s), 146.14 (s), 146.46 (s), 189.37 (s); IR (KBr): $\delta=3435,2959,2865,1527,1466,1442,1413,1384,1372,1363,1340$, 1329, 1318, 1259, 1318, 1259, 1145, 1093, 1055, 805, $760 \mathrm{~cm}-1$; HR-MS (FAB+): found: $m / z=495.2081$, calcd. for $\mathrm{C}_{20} \mathrm{H}_{30} \mathrm{AuN}_{2}[\mathrm{M}-\mathrm{Cl}]^{+}: 495.2075$. 


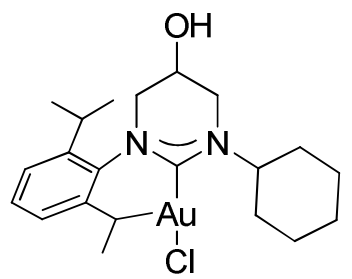

6h was prepared according to GP 2. $100 \quad \mathrm{mg} \quad(238 \quad \mu \mathrm{mol})$ 2,6diisopropylphenylisocyanogold(I) chloride, $137 \mathrm{mg}(715 \mu \mathrm{mol}) \mathbf{4 f}$ and $1.5 \mathrm{ml} \mathrm{NEt}_{3}$ in $5 \mathrm{ml}$ of dry dichloromethane were employed. Purification by column chromatography afforded a colourless crystaline solid; yield: $94 \mathrm{mg}(163 \mu \mathrm{mol}, 69 \%) ;{ }^{1} \mathrm{H} \mathrm{NMR}\left(300 \mathrm{MHz}, \mathrm{CDCl}_{3}\right): \delta=$ 1.02-1.52 (m; 5H), $1.18(\mathrm{~d}, \mathrm{~J}=6.9 \mathrm{~Hz} ; 3 \mathrm{H}), 1.20(\mathrm{~d}, \mathrm{~J}=6.9 \mathrm{~Hz} ; 3 \mathrm{H}), 1.35(\mathrm{~d}, \mathrm{~J}=6.8 \mathrm{~Hz} ; 3 \mathrm{H})$, $1.36(\mathrm{~d}, \mathrm{~J}=6.9 \mathrm{~Hz} ; 3 \mathrm{H}), 1.64-1.88(\mathrm{~m} ; 5 \mathrm{H}), 2.29(\mathrm{~d}, \mathrm{~J}=4.9 \mathrm{~Hz} ; 1 \mathrm{H}), 2.81(\mathrm{~m}, \mathrm{~J}=6.9 \mathrm{~Hz} ; 1$ H), $3.12(\mathrm{~m}, \mathrm{~J}=6.9 \mathrm{~Hz} ; 1 \mathrm{H}), 3.19-3.44(\mathrm{~m} ; 4 \mathrm{H}), 4.33-4.40(\mathrm{~m} ; 1 \mathrm{H}), 4.77-4.87(\mathrm{~m} ; 1 \mathrm{H}), 7.13-$ $7.19(\mathrm{~m} ; 2 \mathrm{H}), 7.33(\mathrm{~m} ; 1 \mathrm{H}) .{ }^{13} \mathrm{C} \mathrm{NMR}\left(75 \mathrm{MHz}, \mathrm{CDCl}_{3}\right): \delta=24.74(\mathrm{q}), 24.83(\mathrm{q}), 24.94(\mathrm{t}, 2$ C), $25.40(\mathrm{t}), 25.43(\mathrm{t}), 25.50(\mathrm{t}), 27.66(\mathrm{~d}), 28.51(\mathrm{~d}), 30.97(\mathrm{t}), 30.97(\mathrm{t}), 45.18(\mathrm{t}), 54.83(\mathrm{t})$, 60.45 (d), 67.71 (d), 124.69 (d), 125.22 (d), 129.66 (d), 141.09 (q), 145.85 (q), 145.94, (q), 190.38 (q); IR (KBr): $v=3450,2961,2931,2862,1626,1590,1527,1452,1384,1361$, 1303, 1267, 1221, 1167, 1104, 1074, 1056, 959, 805, 760; HR-MS (FAB+): $\mathrm{C}_{22} \mathrm{H}_{34} \mathrm{AuClN} \mathrm{N}_{2} \mathrm{O}$ $[\mathrm{M}]^{+}$calc. 574.2025, found 574.2036.

\section{6i}

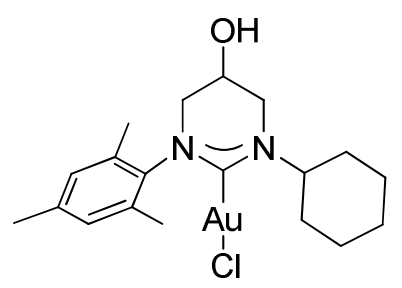

6i was prepared according to GP 2. $96 \mathrm{mg}(254 \mu \mathrm{mol})$ mesitylisocyanogold(I) chloride, 86 $\mathrm{mg}(449 \mu \mathrm{mol})$ 4f and $1.5 \mathrm{ml} \mathrm{NEt}_{3}$ in $10 \mathrm{ml}$ of dry dichloromethane were employed. Purification by column chromatography afforded a colourless crystaline solid; yield: $122 \mathrm{mg}$ $(229 \mu \mathrm{mol}, 90 \%) ;{ }^{1} \mathrm{H}$ NMR $\left(300 \mathrm{MHz}, \mathrm{CDCl}_{3}\right): \delta=1.03-1.13(\mathrm{~m} ; 1 \mathrm{H}), 1.33-1.59(\mathrm{~m} ; 4 \mathrm{H}$, CyH), 1.65-1.69 (m; 1 H), 1.79-1.88 (m; 4 H), 2.15 (s; 3 H), 2.15 (d, J = 5.2 Hz; 1 H), 2.20 (s; $3 \mathrm{H}), 2.25(\mathrm{~s} ; 3 \mathrm{H}), 3.12-3.44(\mathrm{~m} ; 4 \mathrm{H}), 4.35-4.37(\mathrm{~m}, 1 \mathrm{H}), 4.83(\mathrm{tt}, \mathrm{J}=11.6,3.7 \mathrm{~Hz} ; 1 \mathrm{H})$, $6.87(\mathrm{~s} ; 1 \mathrm{H}), 6.89$ (s; $1 \mathrm{H}) .{ }^{13} \mathrm{C} \mathrm{NMR}\left(75 \mathrm{MHz}, \mathrm{CDCl}_{3}\right): \delta=17.69$ (q), 17.77 (q), 21.13 (q), $25.22(\mathrm{t}), 25.23(\mathrm{t}), 25.33(\mathrm{t}), 30.56(\mathrm{t}), 30.80(\mathrm{t}), 45.04(\mathrm{t}), 52.51(\mathrm{t}), 60.23(\mathrm{~d}), 67.56(\mathrm{~d})$, 
129.64 (d), 130.07 (d), 134.78 (s, 2 C), 138.33 (s), 141.24 (s), 189.88 (s); IR (KBr): $v=3448$, $2931,2856,1609,1531,1451,1362,1304,1248,1175,1108,961,894,853,726,583$. HRMS (FAB+, NPOE): $\mathrm{C}_{19} \mathrm{H}_{28} \mathrm{AuClN}_{2} \mathrm{O}[\mathrm{M}]^{+}$calc. 532.1556, found 532.1563 .

6j<smiles>CCc1cccc(CC)c1N1CC(O)CN(C2CCCCC2)C1=[Ge]Cl</smiles>

6j was prepared according to GP 2. $150 \mathrm{mg}$ (383 $\mu \mathrm{mol})$ 2,6-diethylisocyanogold(I) chloride, $84 \mathrm{mg}(440 \mu \mathrm{mol}) \mathbf{4 f}$ and $1.5 \mathrm{ml} \mathrm{NEt}_{3}$ in $10 \mathrm{ml}$ of dry dichloromethane were employed. Purification by column chromatography afforded a colourless crystaline solid; yield: $160 \mathrm{mg}$ $(293 \mu \mathrm{mol}, 76 \%) ; 1 \mathrm{H} \mathrm{NMR}\left(300 \mathrm{MHz}, \mathrm{CDCl}_{3}\right): \delta=1.00-1.53(\mathrm{~m} ; 5 \mathrm{H}), 1.28(\mathrm{t}, \mathrm{J}=7.6 \mathrm{~Hz} ; 6$ H), 1.64-1.88 (m; 5 H), 2.27-2.29 (m; 1 H), 2.37-2.76 (m; 4 H), 3.17-3.46 (m; 4 H), 4.32-4.39 $(\mathrm{m} ; 1 \mathrm{H}), 4.82(\mathrm{tt}, \mathrm{J}=11.5,3.6 \mathrm{~Hz} ; 1 \mathrm{H}), 7.11-7.16(\mathrm{~m} ; 2 \mathrm{H}), 7.29(\mathrm{t}, \mathrm{J}=7.6 \mathrm{~Hz} ; 1 \mathrm{H}) ;{ }^{13} \mathrm{C}$ NMR (75 MHz, $\left.\mathrm{CDCl}_{3}\right): \delta=14.69(\mathrm{q}, 2 \mathrm{C}), 23.32(\mathrm{t}), 23.80$ (t), 25.40 (t, $\left.2 \mathrm{C}\right), 25.50(\mathrm{t})$, $30.70(t), 30.95(t), 45.27(t), 53.83(t), 60.39(d), 67.80(d), 126.69(d), 127.17(d), 129.19$ (d), 140.84 (s), 140.89 (s), 142.75 (s), 190.22 (s); IR (KBr): v =2932, 2857, 1631, 1529, 1456, 1362, 1307, 1264, 1167, 1105, 960, 894, 869, 807, 707, 570; HR-MS (FAB ${ }^{+}$, NPOE): $\mathrm{C}_{20} \mathrm{H}_{30} \mathrm{AuClN}_{2} \mathrm{O}[\mathrm{M}]^{+}$calc. 546.1712, found 546.1697.

\section{8}

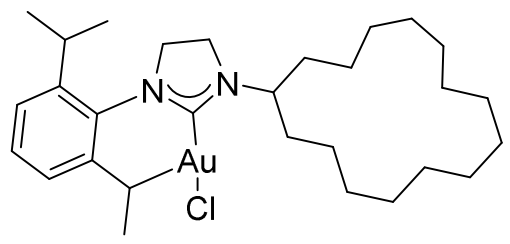

In a three times fire-dried Schlenk-flask filled with nitrogen, the isocyanogold(l) complex (223 $\mathrm{mg}, 531 \mathrm{mmol})$ was dissolved in absolute DCM. The N-(2chloroethyl)cyclopentadecanaminium chloride $(181 \mathrm{mg}, 558 \mu \mathrm{mol})$ and trimethylamine ( 161 $\mathrm{mg}, 1.59 \mathrm{mmol}$ ) were added respectively. The reaction progress was observed by thin layer chromatography $\left(\mathrm{SiO}_{2}, \mathrm{DCM}\right)$. All volatiles were removed under reduced pressure. The 
resulting pale lila residue was finally purified by column chromatography $\left(\mathrm{SiO}_{2}, \mathrm{DCM}\right)$; colorless solid; yield: $323 \mathrm{mg}(481 \mu \mathrm{mol}, 91 \%) ;{ }^{1} \mathrm{H}$ NMR $\left(600 \mathrm{MHz}, \mathrm{CDCl}_{3}\right): \delta=1.22(\mathrm{~d}, J=$ $\left.6.9 \mathrm{~Hz}, 6 \mathrm{H},-\mathrm{CH}_{3}\right), 1.31-1.42\left(\mathrm{~m}, 22 \mathrm{H},-\mathrm{CH}_{2^{-}},-\mathrm{CH}_{3}\right), 1.43-1.49\left(6 \mathrm{H},-\mathrm{CH}_{2}-\right), 1.62(\mathrm{~m}, 2$ $\left.\mathrm{H},-\mathrm{CH}_{2}-\right), 1.71\left(\mathrm{~m}, 4 \mathrm{H},-\mathrm{CH}_{2}-\right), 2.87$ (m, J=6.8 Hz, $\left.2 \mathrm{H},-\mathrm{CH}-\right), 3.74(\mathrm{~m}, J=4.6 \mathrm{~Hz}, 4 \mathrm{H},-$ $\left.\mathrm{CH}_{2^{-}}\right) 4.64\left(\mathrm{~s}, 1 \mathrm{H},-\mathrm{CH}_{2}-\right), 7.2(\mathrm{~d}, J=7.8 \mathrm{~Hz}, 2 \mathrm{H}, \mathrm{ArH}), 7.4$ (t, J=7.8 Hz, $\left.1 \mathrm{H}, \mathrm{ArH}\right)$; ${ }^{13} \mathrm{CNMR}\left(150 \mathrm{MHz}, \mathrm{CDCl}_{3}\right.$ ): $\delta=24.40$ (2C), 24.43 (4 C), 25.18, 26.73 (2 C), 26.79 (2C), 26.96, 27.02, 27.16, 28.76, (2 C) 30.62, 43.53, 52.85, 57.77, 124.67 (1 C) 129.90, 134.77, (2 C) 146.90 (1 C), 194.12 (1 C); IR (KBr): $v=2929,2856,2608,2500,1502,1458,1384,1364$, 1275, 1266, 1106, 1074, 939, 806, 763, 737, 619; HR-MS (FAB+): found: $\mathrm{m} / \mathrm{z}=635.3660$, calcd for $\mathrm{C}_{30} \mathrm{H}_{50} \mathrm{~N}_{2} \mathrm{Au}\left[\mathrm{M}-\mathrm{Cl}^{-}\right]^{+}=635.3640$.

10

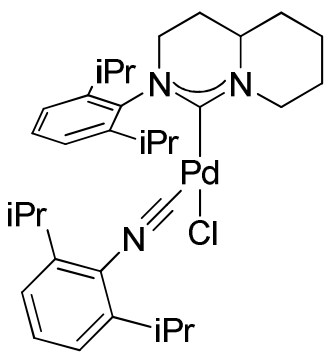

$65.5 \mathrm{mg}(356 \mu \mathrm{mol}) 4 \mathrm{e}$ and $0.5 \mathrm{ml} \mathrm{NEt}{ }_{3}$ were added to a solution of $157 \mathrm{mg}(285 \mu \mathrm{mol})$ cis$\left.\left[\mathrm{PdCl}_{2} \text { (diisopropylphenylisonitrile) }\right)_{2}\right]$ in $8 \mathrm{ml}$ dry THF and the mixture was stirred for $12 \mathrm{~h}$ at RT. After this period, the solvent was removed under reduced pressure and the crude product was dissolved in DCM and washed with saturated $\mathrm{NH}_{4} \mathrm{Cl}$ solution. The organic layer was dried over $\mathrm{Na}_{2} \mathrm{SO}_{4}$, filtered and all volatiles were removed under reduced pressure. The crude product was dissolved in a minimum of DCM and diethyl ether was added, initiating crystallization of $155 \mathrm{mg}(234 \mu \mathrm{mol}, 82 \%, 2.5: 1$ mixture of diastereomeres) $\mathbf{1 0}$.

major isomer: ${ }^{1} \mathrm{H} \mathrm{NMR}\left(500 \mathrm{MHz}, \mathrm{CD}_{2} \mathrm{Cl}_{2}\right): \delta=1.04(\mathrm{~d}, J=6.9 \mathrm{~Hz}, 3 \mathrm{H}), 1.14(\mathrm{~d}, J=6.9$ $\mathrm{Hz}, 3 \mathrm{H}), 1.24-1.29$ (m, 15H), $1.44(\mathrm{~d}, J=6.9 \mathrm{~Hz}, 3 \mathrm{H}), 1.62-2.01(\mathrm{~m}, 6 \mathrm{H}), 2.12-2.19(\mathrm{~m}, 1 \mathrm{H})$, 2.25-2.31 (m, 1H), 3.01 (sept, $J=6.9 \mathrm{~Hz}, 1 \mathrm{H}), 3.24-3.33(\mathrm{~m}, 3 \mathrm{H}), 3.36-3.45$ (m, 2H), 3.52$3.60(\mathrm{~m}, 1 \mathrm{H}), 3.69-3.74(\mathrm{~m}, 1 \mathrm{H}), 5.84-5.87(\mathrm{~m}, 1 \mathrm{H}), 7.22-7.26(\mathrm{~m}, 3 \mathrm{H}), 7.35-7.37(\mathrm{~m}, 1 \mathrm{H})$, 7.44-7.48 (m, $2 \mathrm{H}) ;{ }^{13} \mathrm{C}$ NMR (75 MHz, $\left.\mathrm{CD}_{2} \mathrm{Cl}_{2}\right): \delta=24.04$ (q), 24.78 (q), 24.84 (q, 2C), 25.20 (q), 25.67 (t), 26.31 (q), 27.88 (t), 28.25 (q), 28.67 (q), 28.77 (t), 30.94 (d), 31.05 (d), 31.48 (d), 31.65 (d), $35.58(\mathrm{t}), 50.44(\mathrm{t}), 58.29(\mathrm{~d}), 60.43(\mathrm{t}), 125.25(\mathrm{~s}), 125.87(\mathrm{~d}, 2 \mathrm{C})$, 125.96 (d), 127.19 (d), 131.71 (d), 132.83 (d), 137.27 (s), 142.41 (s), 146.90 (s), 147.97 (s, 
2C), 149.87 (s), 180.84 (s); IR (KBr): $v=3442,2965,2034,2868,2183,1590,1527,1466$, 1442, 1408, 1385, 1364, 1327, 1317, 1276, 1256, 1228, 1092, 802, $750 \mathrm{~cm}^{-1}$; HR-MS $\left(\mathrm{FAB}+\right.$ ): found: $m / z=626.2471$, calcd. for $\mathrm{C}_{33} \mathrm{H}_{47} \mathrm{ClN}_{3} \mathrm{Pd}[\mathrm{M}-\mathrm{Cl}]^{+}: 626.2493$.

\section{General procedure 3 (GP 3) for the synthesis of NAC gold(I) chlorides}

Under an atmosphere of argon the choosen isocyano gold(I) chloride ${ }^{[3]}$ was dissolved in absolute dichloromethane. Afterwards the respective acetal functionalised amine ${ }^{[4]}$ was added. The resulting reaction mixture was stirred at room temperature and the progress of the reaction was monitored by TLC analysis. Reactiontimes were typically over night. After complete conversion of the starting materials was observed the solvent was removed under reduced pressureand the residue was washed with hexanes. If needed, the raw products were further purified by columne chromatography $\left(\mathrm{SiO}_{2} ; \mathrm{DCM} /\right.$ hexanes 1:10 -> 1:1).

\section{5a}

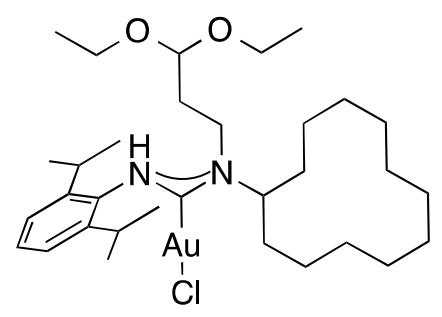

The synthesis was accomplished according to GP3. $200 \mathrm{mg} \quad(477 \mu \mathrm{mol})$ diisopropylphenylisocyanogold(I) chloride and $179 \quad \mathrm{mg} \quad(572 \mu \mathrm{mol}) \quad \mathrm{N}-(3,3-$ diethoxypropyl)cyclododecanamine in $4 \mathrm{~mL}$ DCM were used. colorless solid, yield: $300 \mathrm{mg}$ $(409 \mu \mathrm{mol}, 86 \%) ;{ }^{1} \mathrm{H}$ NMR $\left(300 \mathrm{MHz}, \mathrm{CD}_{2} \mathrm{Cl}_{2}\right) \delta=1.02(\mathrm{t}, \mathrm{J}=7.0 \mathrm{~Hz}, 6 \mathrm{H}), 1.17(\mathrm{~d}, \mathrm{~J}=6.9$ $\mathrm{Hz}, 6 \mathrm{H}), 1.34(\mathrm{~d}, \mathrm{~J}=6.9 \mathrm{~Hz}, 6 \mathrm{H}), 1.33-1.56(\mathrm{~m}, 18 \mathrm{H}), 1.68-1.98(\mathrm{~m}, 4 \mathrm{H}), 2.04(\mathrm{dt}, \mathrm{J}=4.3 \mathrm{~Hz}$, $\mathrm{J}=7.4 \mathrm{~Hz}, 2 \mathrm{H}), 3.12(\mathrm{sep}, \mathrm{J}=6.9 \mathrm{~Hz}, 2 \mathrm{H}), 3.48(\mathrm{t}, \mathrm{J}=7.4 \mathrm{~Hz}, 2 \mathrm{H}), 3.48(\mathrm{dq}, \mathrm{J}=9.3 \mathrm{~Hz}, \mathrm{~J}=$ $7.0 \mathrm{~Hz}, 2 \mathrm{H}$ ), $3.66(\mathrm{dq}, \mathrm{J}=9.3 \mathrm{~Hz}, \mathrm{~J}=7.0 \mathrm{~Hz}, 2 \mathrm{H}$ ), $4.60(\mathrm{t}, \mathrm{J}=4.3 \mathrm{~Hz}, 1 \mathrm{H}$ ), 5.16 (qui, $\mathrm{J}=6.3$ $\mathrm{Hz}, 1 \mathrm{H}, \mathrm{CH}), 7.21$ (d, J = 7.8 Hz, 2H, ArH), 7.39 (t, J = 7.8 Hz, 1H, ArH), 8.41 (s, 1H, NH); ${ }^{13} \mathrm{C}$ NMR (75 MHz, $\mathrm{CD}_{2} \mathrm{Cl}_{2}$ ) $\delta=15.3$ (q, 2C), $22.6(\mathrm{t}), 22.7$ (t, 2C), 23.3 (t, 2C), 23.5 (q, 2C), 24.0 (q, 2C), 24.6 (t, 2C), 24.7 (t, 2C), 28.9 (d, 2C), 29.7 (t, 2C), $34.4(\mathrm{t}), 38.4(\mathrm{t}), 63.5(\mathrm{t}, 2 \mathrm{C})$, 64.1 (d), 101.8 (d), 124.0 (d, 2C), 129.1 (d), 133.7 (s, 2C), 147.1 (s), carbene carbon signal could not be observed; FT-IR (KBr): 3438, 3246, 2930, 2865, 1547, 1488, 1469, 1444, 1425 , 
1383, 1362, 1346, 1281, 1237, 1166, 1121, 1058, 967, 801, 758; HR-MS (FAB $\left.{ }^{+}\right):$found m/z $=732.3702$, calcd. for $\mathrm{C}_{32} \mathrm{H}_{56} \mathrm{AuClN}_{2} \mathrm{O}_{2}[\mathrm{M}]^{+}: 732.3696$.

\section{5b}

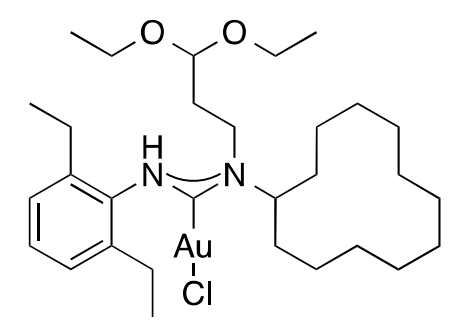

The synthesis was accomplished according to GP3. $100 \mathrm{mg}(255 \mu \mathrm{mol})$ of 2,6diethylphenylisocyanogold(I)chloride as well as $84 \mathrm{mg} \quad(268 \mu \mathrm{mol})$ of $N-(3,3-$ diethoxypropyl)cyclododecanamine were dissolved in $4 \mathrm{~mL}$ of dichloromethane. Colorless solid; yield: $137 \mathrm{mg}(194 \mu \mathrm{mol}, 76 \%) ;{ }^{1} \mathrm{H}$ NMR $\left(300 \mathrm{MHz}, \mathrm{CD}_{2} \mathrm{Cl}_{2}\right) \delta=0.99(\mathrm{~d}, 6 \mathrm{H}, \mathrm{J}=7.0$ $\mathrm{Hz}$ ), $1.22(\mathrm{~d}, 6 \mathrm{H}, \mathrm{J}=7.0 \mathrm{~Hz}$ ), 1.33-1.56 (m, 18H, (overlapping with water peak in the nmr solvent)), 1.68-1.98 (m, 4H), 2.00-2.08 (m, 2H), 2.63 (dt, J = 7.6 Hz, J = 6.9 Hz, 4H), 3.40$3.50(\mathrm{~m}, 3 \mathrm{H}), 3.60-3.72(\mathrm{~m}, 3 \mathrm{H}), 4.60(\mathrm{t}, \mathrm{J}=4.3 \mathrm{~Hz}, 1 \mathrm{H}), 5.16(\mathrm{~m}, 1 \mathrm{H}), 7.21(\mathrm{~d}, \mathrm{~J}=7.8 \mathrm{~Hz}$, 2H, ArH), 7.39 (t, J = 7.8 Hz, 1H, ArH), $8.41(\mathrm{~s}, 1 \mathrm{H}, \mathrm{NH}) ;{ }^{13} \mathrm{C} \mathrm{NMR}\left(75 \mathrm{MHz}, \mathrm{CD}_{2} \mathrm{Cl}_{2}\right) \delta=$ $15.3(\mathrm{q}, 2 \mathrm{C}), 22.6(\mathrm{t}), 22.7(\mathrm{t}, 2 \mathrm{C}), 23.3(\mathrm{t}, 2 \mathrm{C}), 24.0(\mathrm{q}, 2 \mathrm{C}), 24.6(\mathrm{t}, 2 \mathrm{C}), 24.7(\mathrm{t}, 2 \mathrm{C}), 28.9(\mathrm{~d}$, 2C), $29.7(\mathrm{t}, 2 \mathrm{C}), 34.4(\mathrm{t}), 38.4(\mathrm{t}), 63.5(\mathrm{t}, 2 \mathrm{C}), 64.1$ (d), $101.8(\mathrm{~d}), 124.0$ (d, 2C), 129.1 (d), 133.7 (s, 2C), 147.1 (s), 195.8 (s) IR (KBr) n = 3244, 3070, 2930, 2865, 1592, 1549, 1486, 1469, 1443, 1425, 1372, 1347, 1281, 1237, 1199, 1166, 1120, 1058, $966 \mathrm{~cm}^{-1}$; HR-MS $\left(\mathrm{FAB}^{+}\right)$: found: $\mathrm{m} / \mathrm{z}=704.3448$, calcd. for $\mathrm{C}_{30} \mathrm{H}_{52} \mathrm{AuClN}_{2} \mathrm{O}_{2}\left[\mathrm{M}^{+}\right]: 704.3383$; found: $\mathrm{m} / \mathrm{z}=$ 669.3682, calcd. for $\mathrm{C}_{30} \mathrm{H}_{52} \mathrm{AuN}_{2} \mathrm{O}_{2}\left[\mathrm{M}^{+}\right]$: 669.3694 . 


\section{$15 \mathrm{c}$}

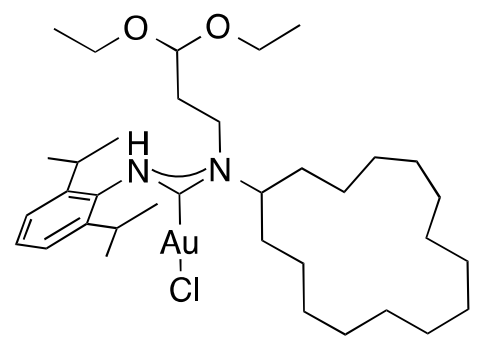

The synthesis was accomplished according to GP3. $150 \mathrm{mg} \quad(357 \mu \mathrm{mol})$ diisopropylphenylisocyanogold(I) chloride and $153 \quad \mathrm{mg} \quad(428 \mu \mathrm{mol}) \quad N-(3,3-$ diethoxypropyl)cyclopentadecanamine in $2 \mathrm{~mL}$ DCM were used. colorless solid; yield: 148 $\operatorname{mg}(191 \mu \mathrm{mol}, 54 \%) ;{ }^{1} \mathrm{H}$ NMR $\left(300 \mathrm{MHz}, \mathrm{CD}_{2} \mathrm{Cl}_{2}\right) \delta=1.02(\mathrm{t}, \mathrm{J}=7.1 \mathrm{~Hz}, 6 \mathrm{H}), 1.17(\mathrm{~d}, \mathrm{~J}=$ $6.8 \mathrm{~Hz}, 6 \mathrm{H}, \mathrm{CH} 3), 1.34$ (d, J = 6.8 Hz, 6H), 1.21-1.83 (m, 28H), 2.04 (dt, J = 3.8 Hz, J = 7.7 $\mathrm{Hz}, 2 \mathrm{H}$ ), 3.12 (seq, J = 6.8 Hz, 2H, CH), 3.46 (d, J = 7.7 Hz, 2H), 3.48 (dq, J = 9.2 Hz, J = 7.1 $\mathrm{Hz}, 2 \mathrm{H}), 3.67$ (dq, J = 9.2 Hz, J = 7.1 Hz, 2H), 4.61 (t, J = 3.8 Hz, 1H), 5.02 (qui, J = 6.9 Hz, 1H), $7.21(\mathrm{t}, \mathrm{J}=7.6 \mathrm{~Hz}, 2 \mathrm{H}, \mathrm{ArH}), 7.38(\mathrm{~d}, \mathrm{~J}=7.6 \mathrm{~Hz}, 1 \mathrm{H}, \mathrm{ArH}), 8.44(\mathrm{~s}, 1 \mathrm{H}, \mathrm{NH}) ;{ }^{13} \mathrm{C} \mathrm{NMR}$ $\left(75 \mathrm{MHz}, \mathrm{CD}_{2} \mathrm{Cl}_{2}\right) \delta=15.3(\mathrm{q}, 2 \mathrm{C}), 23.4$ (q, 2C), 24.0 (q, 2C), 24.9 (t, 2C), 27.2 (t, 2C), 27.3 $(\mathrm{t}, 4 \mathrm{C}), 27.4(\mathrm{t}, 2 \mathrm{C}), 27.5(\mathrm{t}, 2 \mathrm{C}), 28.9(\mathrm{~d}, 2 \mathrm{C}), 32.0(\mathrm{t}, 2 \mathrm{C}), 34.2(\mathrm{t}), 38.0(\mathrm{t}), 63.5(\mathrm{t}, 2 \mathrm{C}), 67.0$ (d), 101.8 (d), 124.0 (d, 2C), 129.2 (d), 136.0 (s), 147.1 (s, 2C), 194.1 (s); FT-IR (KBr): 3434, 3250, 3068, 2926, 2857, 1591, 1548, 1488, 1458, 1425, 1383, 1340, 1233, 1120, 1028, 970, 936, 800, 757, 707; HR-MS $\left(\mathrm{FAB}^{+}\right)$: found $\mathrm{m} / \mathrm{z}=774.4153$, calcd. for $\mathrm{C}_{35} \mathrm{H}_{62} \mathrm{AuClN}_{2} \mathrm{O}_{2}$ $[\mathrm{M}]^{+}: 774.4165$.

\section{5d}

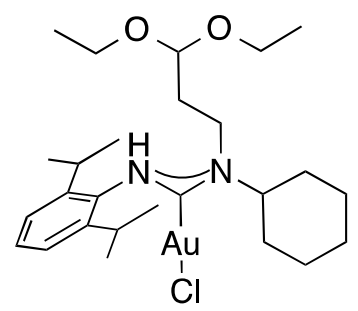

The synthesis was accomplished according to GP3. $153 \mathrm{mg}(363 \mu \mathrm{mol})$ of 2,6diisopropylphenylisocyanogold(I) chloride and $100 \mathrm{mg} \quad(436 \mu \mathrm{mol})$ of $\mathrm{N}-(3,3-$ diethoxypropyl)cyclohexanamine and $2 \mathrm{~mL}$ DCM were used. 
colorless solid; yield: $117 \mathrm{mg}(181 \mu \mathrm{mol}, 50 \%) ;{ }^{1} \mathrm{H}$ NMR $\left(300 \mathrm{MHz}, \mathrm{CD}_{2} \mathrm{Cl}_{2}\right) \delta=1.07(\mathrm{t}, \mathrm{J}=$ $7.1 \mathrm{~Hz}, 6 \mathrm{H}), 1.22$ (d, J = 6.9 Hz, 6H), 1.38 (d, J = 6.9 Hz, 6H), 1.41-1.81 (m, 6H), 1.93 (pt, J $=11.7 \mathrm{~Hz}, 4 \mathrm{H}), 2.06(\mathrm{dt}, \mathrm{J}=3.7 \mathrm{~Hz}, \mathrm{~J}=7.7 \mathrm{~Hz}, 2 \mathrm{H}), 3.16(\mathrm{sep}, \mathrm{J}=6.9 \mathrm{~Hz}, 2 \mathrm{H}), 3.55(\mathrm{t}, \mathrm{J}=$ $7.7 \mathrm{~Hz}, 2 \mathrm{H}), 3.53(\mathrm{dq}, \mathrm{J}=9.2 \mathrm{~Hz}, \mathrm{~J}=7.1 \mathrm{~Hz}, 2 \mathrm{H}), 3.71(\mathrm{dq}, \mathrm{J}=9.2 \mathrm{~Hz}, \mathrm{~J}=7.1 \mathrm{~Hz}, 2 \mathrm{H}), 4.66$ $(\mathrm{t}, \mathrm{J}=4.4 \mathrm{~Hz}, 1 \mathrm{H}), 4.84(\mathrm{tt}, \mathrm{J}=11.7 \mathrm{~Hz}, \mathrm{~J}=3.4 \mathrm{~Hz}, 1 \mathrm{H}), 7.26(\mathrm{~d}, \mathrm{~J}=7.7 \mathrm{~Hz}, 2 \mathrm{H}, \mathrm{ArH}), 7.43$ (t, J = 7.7 Hz, 1H, ArH), $8.46(\mathrm{~s}, 1 \mathrm{H}, \mathrm{NH}) ;{ }^{13} \mathrm{C} \mathrm{NMR}\left(75 \mathrm{MHz}, \mathrm{CD}_{2} \mathrm{Cl}_{2}\right) \delta=15.3$ (q, 2C), $23.4(\mathrm{q}, 2 \mathrm{C}), 24.0(\mathrm{q}, 2 \mathrm{C}), 25.6(\mathrm{t}), 25.9(\mathrm{t}, 2 \mathrm{C}), 28.8(\mathrm{~d}, 2 \mathrm{C}), 32.1(\mathrm{t}, 2 \mathrm{C}), 34.9(\mathrm{t}), 38.6(\mathrm{t})$, 63.3 (t, 2C), 68.4 (d), 101.6 (d), 124.0 (d, 2C), 129.2 (d), 135.9 (s), 147.1 (s, 2C), 193.4 (s); FT-IR (KBr): 3446, 3245, 2964, 2931, 2865, 1633, 1592, 1547, 1493, 1447, 1426, 1384, 1348, 1300, 1235, 1124, 1059, 998, 802, 758; HR-MS $\left(\mathrm{FAB}^{+}\right)$: found $\mathrm{m} / \mathrm{z}=648.2770$, calcd. for $\mathrm{C}_{26} \mathrm{H}_{44} \mathrm{AuClN}_{2} \mathrm{O}_{2}[\mathrm{M}]^{+}:$648.2757; found $\mathrm{m} / \mathrm{z}=613.3078$, calcd. for $\mathrm{C}_{26} \mathrm{H}_{44} \mathrm{AuN}_{2} \mathrm{O}_{2}[\mathrm{M}]^{+}$: 613.3068 .

$15 \mathrm{e}$

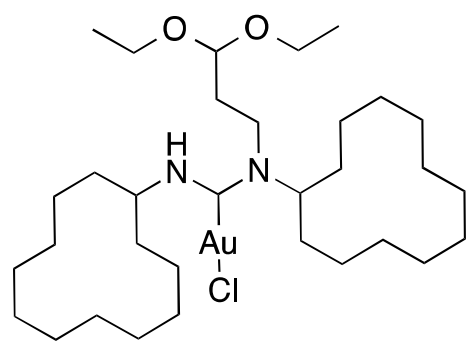

The synthesis was accomplished according to GP3. $100 \mathrm{mg} \quad(234 \mu \mathrm{mol})$ diisopropylphenylisocyanogold(I) chloride and $77 \quad \mathrm{mg} \quad(247 \mu \mathrm{mol}) \quad N-(3,3-$ diethoxypropyl)cyclopentadecanamine in $3 \mathrm{~mL}$ DCM were used, colorless solid; yield: 151 mg, (204 $\mu \mathrm{mol}, 87 \%) ;{ }^{1} \mathrm{H}$ NMR $\left(\mathrm{CDCl}_{3}, 400 \mathrm{MHz}\right): \delta=1.21$ (t, J = 7.9Hz, 6H), 1.24-1.62 (m, 39H), 1.63- 1.82 (m, 7H), 3.12-3.21 (m, 2H), 3.47-3.55 (m, 2H), 3.59-3.69 (m, 2H), 4.47 (t, J $=4.7 \mathrm{~Hz}, 1 \mathrm{H}), 4.45-4.50(\mathrm{~m}, 1 \mathrm{H}), 5.04-5.13(\mathrm{~m}, 1 \mathrm{H}), 6.36(\mathrm{~d}, \mathrm{~J}=8.4 \mathrm{~Hz}, 1 \mathrm{H},-\mathrm{NH}) ;{ }^{13} \mathrm{C}$ NMR ( $\left.\mathrm{CDCl}_{3}, 100 \mathrm{MHz}\right): \delta=15.59(2 \mathrm{C}, \mathrm{q}), 21.57(2 \mathrm{C}, \mathrm{t}), 22.32(\mathrm{t}), 22.47(2 \mathrm{C}, \mathrm{t}), 23.21(2 \mathrm{C}$, t), $23.61(2 \mathrm{C}, \mathrm{t}), 23.72(2 \mathrm{C}, \mathrm{t}), 23.78(\mathrm{t}), 24.22(2 \mathrm{C}, \mathrm{t}), 24.37(2 \mathrm{C}, \mathrm{t}), 24.44(2 \mathrm{C}, \mathrm{t}), 29.48(2 \mathrm{C}$, t), $31.40(2 \mathrm{C}, \mathrm{t}), 32.94(2 \mathrm{C}, \mathrm{t}), 37.96(\mathrm{t}), 58.22$ (d), $62.87(2 \mathrm{C}, \mathrm{t}), 64.42$ (d), 100.97 (d), 190.10 (s); IR (KBr): $v=3310,2931,2862,1556,1492,1469,1444,1349,1244,1230,1202,1163$, 1121, 1059, 966, $717 \mathrm{~cm}^{-1}$; HR-MS $\left(\mathrm{FAB}^{+}\right)$: found: $\mathrm{m} / \mathrm{z}=703.4426$, calcd. for $\left[{\left.\mathrm{M}-\mathrm{Cl}^{-}\right]}^{-}\right.$ $\mathrm{C}_{32} \mathrm{H}_{62} \mathrm{AuN}_{2} \mathrm{O}_{2}$ 703.4477, found: $\mathrm{m} / \mathrm{z}=693.3829$, calcd. for [M-OEt $] \mathrm{C}_{30} \mathrm{H}_{57} \mathrm{AuClN}_{2} \mathrm{O}_{2}$ 693.3819. 


\section{General procedure 4 (GP 4) for the synthesis of NHC gold(I) chlorides}

The desired acetal functionalised NAC gold(I) chloride was dissolved in wet dichloromethane. Afterwards 4M hydrochloric acid in Dioxane was added. The resulting reaction mixture was stirred at room temperature under ambient conditions. The progress of the reaction was controlled by TLC analysis. After complete conversion of the starting materials all voltalies were removed under reduced pressure and the resulting residue was purified by column chromatography $\left(\mathrm{SiO}_{2} ; \mathrm{DCM} /\right.$ hexanes 1:10 -> 1:1).

\section{6a}

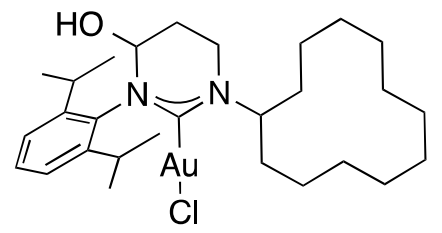

The synthesis of this compound was achieved according to GP4. Herefore $300 \mathrm{mg}$ (409 $\mu \mathrm{mol})$ 15a, $0.3 \mathrm{~mL}(1.20 \mathrm{mmol}) 4 \mathrm{M}$ hydrochloric acid in dioxane and $4 \mathrm{~mL}$ DCM were employed; colorless solid; yield: $121 \mathrm{mg}(184 \mu \mathrm{mol}, 45 \%) ;{ }^{1} \mathrm{H} \mathrm{NMR}\left(300 \mathrm{MHz}, \mathrm{CD}_{2} \mathrm{Cl}_{2}\right) \delta=$ 1.16- 2.03 (m, 35H), 2.23 (ddd, $\mathrm{J}=13.8 \mathrm{~Hz}, \mathrm{~J}=3.0 \mathrm{~Hz}, \mathrm{~J}=2.2 \mathrm{~Hz}, 1 \mathrm{H}), 2.71(\mathrm{sep}, \mathrm{J}=6.9$ $\mathrm{Hz}, 1 \mathrm{H}), 2.80(\mathrm{dd}, \mathrm{J}=4.6 \mathrm{~Hz}, \mathrm{~J}=0.9 \mathrm{~Hz}, 1 \mathrm{H}, \mathrm{OH}), 3.09(\mathrm{sep}, \mathrm{J}=6.9 \mathrm{~Hz}, 1 \mathrm{H}), 3.26-3.50$ (m, 2H), 4.78-4.89 (m, 1H), 5.12-5.25 (m, 1H), 7.25 (ddd, J = 7.7 Hz, J = 3.8 Hz, J = 1.6 Hz, 2H, $\mathrm{ArH}), 7.43(\mathrm{~d}, \mathrm{~J}=7.7 \mathrm{~Hz}, 1 \mathrm{H}, \mathrm{ArH}) ;{ }^{13} \mathrm{C} \mathrm{NMR}\left(75 \mathrm{MHz}, \mathrm{CD}_{2} \mathrm{Cl}_{2}\right) \delta=22.4(\mathrm{t}), 22.6(\mathrm{t}), 22.7$ $(\mathrm{t}), 23.2(\mathrm{q}, 2 \mathrm{C}), 23.3(\mathrm{q}, 2 \mathrm{C}), 23.9(\mathrm{t}), 24.0(\mathrm{t}), 24.2(\mathrm{t}), 24.7(\mathrm{t}), 25.2(\mathrm{t}), 25.5(\mathrm{t}), 27.2(\mathrm{~d})$, 28.3 (d), 28.6 (t), 28.9 (t), 30.1 (d), 34.6 (d), 64.3 (d), 78.2 (d), 125.0 (d), 125.4 (d), 129.8 (d), 140.3 (s), 146.2 (s), 146.6 (s), 190.9 (s); FT-IR (KBr): 3419, 2929, 2863, 1523, 1469, 1453, 1444, 1414, 1383, 1362, 1299, 1266, 1245, 1221, 1208, 1090, 1055, 982, 970, 803; HR-MS $\left(\mathrm{FAB}^{+}\right)$: found $\mathrm{m} / \mathrm{z}=658.2974$, calcd. for $\mathrm{C}_{28} \mathrm{H}_{46} \mathrm{AuClN}_{2} \mathrm{O}[\mathrm{M}]^{+}:$658.2964. 


\section{$16 b$}

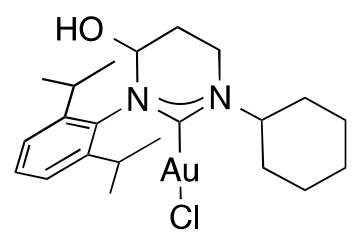

The synthesis of this compound was achieved according to GP4. $100 \mathrm{mg}(154 \mu \mathrm{mol}) \mathbf{1 5 c}, 0.3$ $\mathrm{mL}(1.20 \mathrm{mmol}) 4 \mathrm{M}$ hydrochloric acid in dioxane and $2 \mathrm{~mL}$ DCM were used.

colorless solid; yield: $60 \mathrm{mg}(108 \mu \mathrm{mol}, 70 \%) ;{ }^{1} \mathrm{H}$ NMR $\left(300 \mathrm{MHz}, \mathrm{CD}_{2} \mathrm{Cl}_{2}\right) \delta=1.23(\mathrm{~d}, \mathrm{~J}=$ $6.7 \mathrm{~Hz}, 3 \mathrm{H}), 1.24(\mathrm{~d}, \mathrm{~J}=6.7 \mathrm{~Hz}, 3 \mathrm{H}), 1.36(\mathrm{~d}, \mathrm{~J}=6.7 \mathrm{~Hz}, 3 \mathrm{H}),, 1.41(\mathrm{~d}, \mathrm{~J}=6.7 \mathrm{~Hz}, 3 \mathrm{H}), 1.01-$ $1.79(\mathrm{~m}, 8 \mathrm{H}), 2.01-1.82(\mathrm{~m}, 2 \mathrm{H}), 1.97$ (dt, J = 13.4 Hz, J = 3.6 Hz, 1H), 2.24 (pdq, J = 13.4 $\mathrm{Hz}, \mathrm{J}=2.7 \mathrm{~Hz}, 1 \mathrm{H}), 2.69(\mathrm{sep}, \mathrm{J}=6.8 \mathrm{~Hz}, 1 \mathrm{H}), 2.78(\mathrm{~d}, \mathrm{~J}=4.8 \mathrm{~Hz}, 1 \mathrm{H}, \mathrm{OH}), 3.09$ (sep, J = $6.8 \mathrm{~Hz}, 1 \mathrm{H}), 3.34$ (td, J = 13.4 Hz, J = 3.6 Hz, 1H), 3.52 (pdt, J = 13.4 Hz, J = 3.7 Hz, 1H), $4.84(\mathrm{tt}, \mathrm{J}=11.9 \mathrm{~Hz}, \mathrm{~J}=4.0 \mathrm{~Hz}, 1 \mathrm{H}), 7.25(\mathrm{~d}, \mathrm{~J}=7.7 \mathrm{~Hz}, 2 \mathrm{H}, \operatorname{ArH}), 4.90-4.77(\mathrm{~m}, 1 \mathrm{H}, \mathrm{CH})$, 7.44 (t, J = 7.7 Hz, 1H, ArH), ${ }^{13} \mathrm{C} \operatorname{NMR}\left(75 \mathrm{MHz}, \mathrm{CD}_{2} \mathrm{Cl}_{2}\right) \delta=24.2$ (q), 24.7 (q), 25.1 (q), $25.5(\mathrm{q}), 25.6(\mathrm{t}), 25.7(\mathrm{t}), 25.9(\mathrm{t}), 28.2(\mathrm{~d}), 28.5(\mathrm{~d}), 28.9(\mathrm{t}), 30.9(\mathrm{t}), 31.1(\mathrm{t}), 34.7(\mathrm{t}), 68.9$ (d), 78.2 (d), 125.0 (d), 125.4 (d), 129.8 (d), 140.5 (s), 146.3 (s), 148.4 (s), 190.3 (s); FT-IR (KBr): 3429, 2931, 2862, 1627, 1523, 1452, 1411, 1383, 1358, 1299, 1265, 1207, 1157, 1091, 11056, 1002, 978, 894, 803, 759; HR-MS $\left(\mathrm{FAB}^{+}\right)$: found $\mathrm{m} / \mathrm{z}=574.2045$, calcd. for $\mathrm{C}_{22} \mathrm{H}_{34} \mathrm{AuClN}_{2} \mathrm{O}[\mathrm{M}]^{+}: 574.2025$.

\section{$16 \mathrm{c}$}

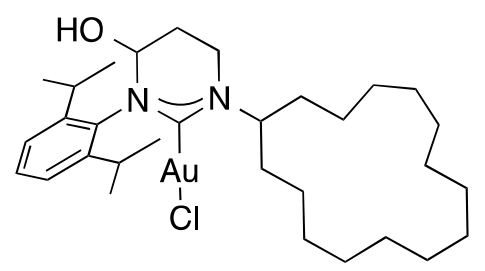

The synthesis of this compound was acomplished according to GP4. Herefore $112 \mathrm{mg}$ (144 $\mu \mathrm{mol})$ 15c, $0.3 \mathrm{~mL}(1.20 \mathrm{mmol}) 4 \mathrm{M}$ hydrochloric acid in dioxane and $4 \mathrm{~mL}$ DCM were used; colorless solid; yield: $31 \mathrm{mg}(45 \mu \mathrm{mol}, 32 \%) ;{ }^{1} \mathrm{H}$ NMR $\left(300 \mathrm{MHz}, \mathrm{CD}_{2} \mathrm{Cl}_{2}\right) \delta=1.22-1.79(\mathrm{~m}$, 39H), 1.95 (ptt, J = 13.8 Hz, J = 3.5 Hz, 1H), 2.24 (dtd, J = 13.8 Hz, J = 2.9 Hz, J = 2.2 Hz, 1H), $2.69(\operatorname{sep}, J=6.9 \mathrm{~Hz}, 1 \mathrm{H}), 2.83(\mathrm{dd}, \mathrm{J}=4.6 \mathrm{~Hz}, \mathrm{~J}=0.9 \mathrm{~Hz}, 1 \mathrm{H}), 2.95-3.03(\mathrm{~m}, 1 \mathrm{H}), 3.10$ $(\mathrm{sep}, \mathrm{J}=6.9 \mathrm{~Hz}, 1 \mathrm{H}), 3.23-3.49(\mathrm{~m}, 2 \mathrm{H}), 4.81-4.88(\mathrm{~m}, 1 \mathrm{H}), 5.13-4.79(\mathrm{~m}, 1 \mathrm{H}), 7.25$ (pd, J = 
$7.7 \mathrm{~Hz}, 2 \mathrm{H}, \mathrm{ArH}), 7.44(\mathrm{t}, \mathrm{J}=7.7 \mathrm{~Hz}, 1 \mathrm{H}, \mathrm{ArH}) ;{ }^{13} \mathrm{C} \mathrm{NMR}\left(75 \mathrm{MHz}, \mathrm{CD}_{2} \mathrm{Cl}_{2}\right) \delta=23.3$ (q, 2C), $24.0(\mathrm{t}), 24.2(\mathrm{t}), 24.6(\mathrm{t}), 24.7(\mathrm{t}), 25.0(\mathrm{q}, 2 \mathrm{C}), 25.1(\mathrm{t}), 25.5(\mathrm{t}), 27.1(\mathrm{t}, 2 \mathrm{C}), 27.3(\mathrm{t}$, 2C), $27.3(\mathrm{t}), 27.5(\mathrm{t}), 27.6(\mathrm{t}), 28.2(\mathrm{~d}), 28.5(\mathrm{~d}), 28.9(\mathrm{t}), 30.2(\mathrm{t}), 31.5(\mathrm{t}), 67.5(\mathrm{~d}), 78.2(\mathrm{~d})$, 124.9 (d), 125.4 (d), 129.8 (d), 140.4 (s), 146.2 (s), 148.5 (s), 191.2 (s), ; FT-IR (KBr): 3451, 3414, 2963, 2925, 2863, 1526, 1467, 1450, 1414, 1382, 1361, 1322, 1298, 1262, 1096, 1054, 1022, 973, 871, 802. HR-MS $\left(\mathrm{FAB}^{+}\right): \mathrm{m} / \mathrm{z}=700.3453$, calcd. for $\mathrm{C}_{31} \mathrm{H}_{52} \mathrm{AuClN}_{2} \mathrm{O}[\mathrm{M}]^{+}$: 700.3434 .

\section{Procedure for the synthesis of 16d and 19a}

In a three times fired-dried Schlenk-flask filled with nitrogen $\mathbf{1 6 b}(150 \mathrm{mg}, 0.21 \mathrm{mmol})$ was dissolved in absolute DCM $(8 \mathrm{ml})$. Then $2 \mathrm{ml}$ of a fresh hydrogen chloride solution (4M in dioxane) were added. The conversion of the starting materials was observed via TLC. After $30 \mathrm{~h}$ it appeared that any further conversion is happening and all volatiles were removed under reduced pressure. The resulting residue which consisted of the two reaction products was purified and seperated by quick columne chromatography under inert atmosphere $\left(\mathrm{SiO}_{2}\right.$, dry DCM).

\section{6d}

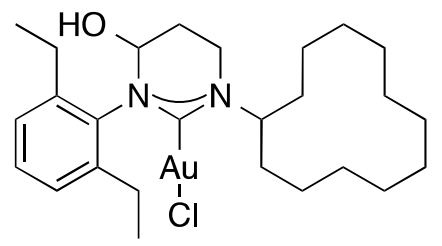

colorless solid; yield: $70 \mathrm{mg}(0.11 \mathrm{mmol}, 52 \%) ;{ }^{1} \mathrm{H}$ NMR $\left(300 \mathrm{MHz}, \mathrm{CD}_{2} \mathrm{Cl}_{2}\right) \delta=1.32(\mathrm{t}, 3 \mathrm{H}$, $\mathrm{J}=7.6 \mathrm{~Hz}), 1.33(\mathrm{t}, 3 \mathrm{H}, \mathrm{J}=7.6 \mathrm{~Hz}), 1.20-1.68(\mathrm{~m}, 18 \mathrm{H}), 1.73-1.90(\mathrm{~m}, 4 \mathrm{H}), 2.17-2.28(\mathrm{~m}$, $1 \mathrm{H}), 2.31-2.83(\mathrm{~m}, 5 \mathrm{H}), 2.85(\mathrm{bs}, 1 \mathrm{H}), 3.26(\mathrm{~m}, 2 \mathrm{H}), 4.84-4.89(\mathrm{~m}, 1 \mathrm{H}), 5.12-5.25(\mathrm{~m}, 1 \mathrm{H})$, 7.21-7.28 (m, 2H, ArH), 7.40 (t, 1H, J = 7.9 Hz, ArH); ${ }^{13} \mathrm{C} \mathrm{NMR}\left(75 \mathrm{MHz}, \mathrm{CD}_{2} \mathrm{Cl}_{2}\right) \delta=14.25$ $(\mathrm{q}), 14.67(\mathrm{q}), 22.11(\mathrm{t}), 22.31(\mathrm{t}), 22.39(\mathrm{t}), 22.86(\mathrm{t}), 22.99(\mathrm{t}), 23.63(\mathrm{t}), 23.81(\mathrm{t}), 23.91(\mathrm{t})$, $24.83(\mathrm{t}), 25.03(\mathrm{t}), 27.01(\mathrm{t}), 27.06(\mathrm{t}), 27.79(\mathrm{t}), 29.66(\mathrm{t}), 34.28(\mathrm{t}), 64.05(\mathrm{~d}), 77.42(\mathrm{~d})$, 126.57 (d), 126.87 (d), 129.01 (d), 140.82 (s), 141.77 (s), 143.29 (s), 190.71 (s); IR (KBr): n = 2928, 2861, 1524, 1469, 1414, 1353, 1300, 1266, 1244, 1204, 1166, 1130, 1095, 1026, 991, 967, 808, $713 \mathrm{~cm}^{-1}$; HR-MS $\left(\mathrm{FAB}^{+}\right)$: found: $\mathrm{m} / \mathrm{z}=630.2669$, calcd. for $\mathrm{C}_{26} \mathrm{H}_{42} \mathrm{AuClN}_{2} \mathrm{O}$

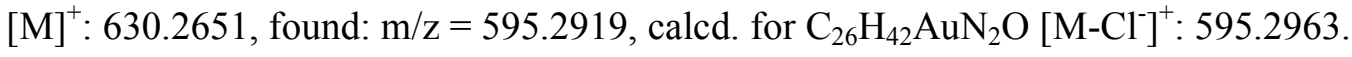


<smiles>CCc1cccc(CC)c1N1C(=O)CCN(C2CCCCCCCCCC2)C1=[Al]Cl</smiles>

In a three times fire-dried Schlenk-flask filled with nitrogen, the $\mathbf{1 6 d}$ (70 $\mathrm{mg}, 0.11 \mathrm{mmol})$ was dissolved in absolute DCM. This solution was cooled to $0{ }^{\circ} \mathrm{C}$ with aid of an ice bath. After the temperature was reached DMP (71 $\mathrm{mg}, 0.17 \mathrm{mmol})$ was added. The resulting colorless suspension was stirred for 5 hours at $0{ }^{\circ} \mathrm{C}$. The reactionprogress was observed by thin layer chromatography $\left(\mathrm{SiO}_{2}, \mathrm{DCM}\right)$. Afterwards the ice bath was allowed to warm up to room temperature. Now, all volatiles were removed under reduced pressure. The resulting pale lila residue was finally purified by columne chromatography $\left(\mathrm{SiO}_{2}, \mathrm{DCM}\right)$; colorless solid; yield: $40 \mathrm{mg}(0.064 \mathrm{mmol}, 58 \%) ;{ }^{1} \mathrm{H} \mathrm{NMR}\left(600 \mathrm{MHz}, \mathrm{CD}_{2} \mathrm{Cl}_{2}\right) \delta=1.24$ (t, 6H, J = 7.5 Hz), 1.29$1.47(\mathrm{~m}, 12 \mathrm{H}), 1.48-1.56(\mathrm{~m}, 4 \mathrm{H}), 1.57-1.64(\mathrm{~m}, 2 \mathrm{H}), 1.71-1.78(\mathrm{~m}, 2 \mathrm{H}), 1.88-1.95(\mathrm{~m}, 2 \mathrm{H})$, $2.37(\mathrm{dq}, 2 \mathrm{H}, \mathrm{J}=15.4 \mathrm{~Hz}, 7.5 \mathrm{~Hz}), 2.51(\mathrm{dq}, 2 \mathrm{H}, \mathrm{J}=15.4 \mathrm{~Hz}, 7.5 \mathrm{~Hz}), 2.85$ (t, 2H, J = 7.2 Hz), 3.71 (t, 2H, J = 7.2 Hz), 5.31-5.42 (m, 1H), 7.25 (d, 2H, J = 8.5 Hz, ArH), 7.44 (t, 1H, J $=8.5 \mathrm{~Hz}, \mathrm{ArH}) ;{ }^{13} \mathrm{C} \mathrm{NMR}\left(75 \mathrm{MHz}, \mathrm{CD}_{2} \mathrm{Cl}_{2}\right) \mathrm{d}=13.71(2 \mathrm{C}, \mathrm{q}), 22.03(2 \mathrm{C}, \mathrm{t}), 22.16(\mathrm{t}), 22.77$ $(2 \mathrm{C}, \mathrm{t}), 23.97(2 \mathrm{C}, \mathrm{t}), 24.21(4 \mathrm{C}, \mathrm{t}), 26.95(\mathrm{t}), 28.26(\mathrm{t}), 30.23(\mathrm{t}), 40.35(\mathrm{t}), 65.81(\mathrm{~d}), 126.51$ (2C, d), 129.82 (d), 137.44 (s), 140.92 (2C, s), 165.92 (s), 197.56 (s); IR (KBr): n = 2930, 2862, 1735, 1636, 1534, 1469, 1411, 1370, 1300, 1277, 1208, 1152, 1121, 1100, 1056, 992, 881, 808, $719 \mathrm{~cm}^{-1}$; HR-MS $\left(\mathrm{FAB}^{+}\right)$: found: $\mathrm{m} / \mathrm{z}=628.2499$, calcd. for $\mathrm{C}_{26} \mathrm{H}_{40} \mathrm{AuClN}_{2} \mathrm{O}$

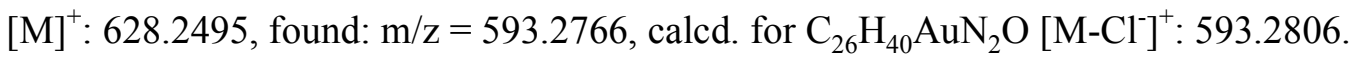

\section{9a}

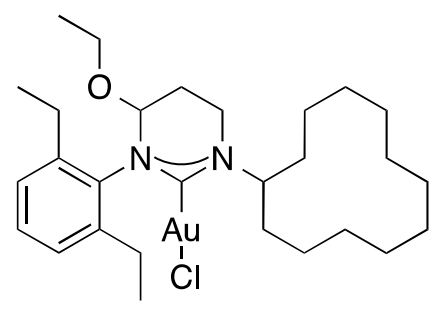

colorless solid; yield $30 \mathrm{mg}(0.045 \mathrm{mmol}, 32 \%) ;{ }^{1} \mathrm{H} \mathrm{NMR}\left(300 \mathrm{MHz}, \mathrm{CD}_{2} \mathrm{Cl}_{2}\right) \delta=1.01$ (t, 
$3 \mathrm{H}, \mathrm{J}=7.0 \mathrm{~Hz}$ ), 1.32 (t, 3H, J = 7.0 Hz), 1.33 (t, 3H, J = 7.0 Hz), 1.18-1.69 (m, 18H), 1.74 $1.89(\mathrm{~m}, 4 \mathrm{H}), 2.19-2,25(\mathrm{~m}, 1 \mathrm{H}), 2.33-2.89(\mathrm{~m}, 5 \mathrm{H}), 3.20$ (q, 2H, J = 7.0 Hz), $3.27(\mathrm{~m}, 2 \mathrm{H})$, $4.41(\mathrm{~s}, 1 \mathrm{H}), 5.11-5.23(\mathrm{~m}, 1 \mathrm{H}), 7.20-7.24$ (m, 2H, ArH), 7.38 (d, 1H, J = 7.6 Hz, ArH); ${ }^{13} \mathrm{C}$ $\operatorname{NMR}\left(75 \mathrm{MHz}, \mathrm{CD}_{2} \mathrm{Cl}_{2}\right) \mathrm{d}=14.26(2 \mathrm{C}, \mathrm{q}), 15.06(\mathrm{q}), 22.11(\mathrm{t}), 22.31(\mathrm{t}), 22.37(\mathrm{t}), 22.85(\mathrm{t})$, $22.99(\mathrm{t}), 23.62(\mathrm{t}), 23.77(\mathrm{t}), 23.79(\mathrm{t}), 23.93(\mathrm{t}), 24.85(\mathrm{t}), 25.05(\mathrm{t}), 26.32(\mathrm{t}), 27.01(\mathrm{t}), 27.26$ (t), $29.68(\mathrm{t}), 34.51(\mathrm{t}), 63.97$ (d), 65.18 (t), 84.56 (d), 126.23 (d), 126.42 (d), 128.69 (d), 140.24 (s), 142.94 (s), 143.49 (s), 190.57 (s); IR (KBr): n = 2928, 2862, 1591, 1523, 1470, $1407,1372,1347,1297,1245,1223,1209,1169,1114,1080,982,887,802,720 \mathrm{~cm}^{-1}$; HR-

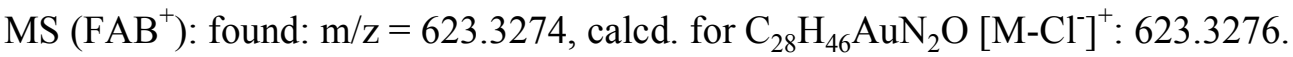

\section{General Procedure 5 (GP 5) for the synthesis of unsaturated sixmembered NHC gold (I) chlorides}

In a carefully fire-dried Schlenk-flask filled with nitrogen the desired acetal functionalised NAC Gold(I) complex $(150 \mu \mathrm{mol})$ was dissolved in absolute toluene $(0.1 \mathrm{M})$. To this solution carefully dried molecular sieve (4 $\AA, 300 \mathrm{mg}$ ) and $0.70 \mathrm{~g}$ Amberlyst 15 were added. The resulting solution was stirred afterwards for 17 hours under reduced pressure (200 mbar). After this time full conversion was checked via TLC. Than the reaction mixture was diluted with absolute DCM and filtered quickly through a pad of $\mathrm{SiO}_{2}$. After this all volatiles were removed under reduced pressure to and the desired complexes were obtained as colorless solids.

$17 \mathbf{a}$

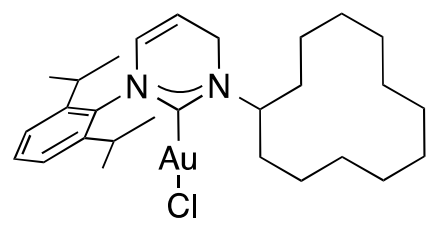

colorless solid; yield: $73.1 \mathrm{mg}, 76 \%$; ${ }^{1} \mathrm{H}$ NMR $\left(500 \mathrm{MHz}, \mathrm{CD}_{2} \mathrm{Cl}_{2}\right): \delta=1.10(\mathrm{~d}, \mathrm{~J}=7.0 \mathrm{~Hz}$, $6 \mathrm{H},-\mathrm{CH} 3), 1.37(\mathrm{~d}, \mathrm{~J}=7.0 \mathrm{~Hz}, 6 \mathrm{H}), 1.20-1.42(\mathrm{~m}, 13 \mathrm{H}), 1.50-1.63(\mathrm{~m}, 5 \mathrm{H}), 1.77-1.85$ (m, 4H), 3.00- $3.06(\mathrm{~m}, 2 \mathrm{H}), 3.95(\mathrm{dd}, \mathrm{J}=3.6 \mathrm{~Hz}, \mathrm{~J}=1.7 \mathrm{~Hz}, 2 \mathrm{H}), 5.16-5.23(\mathrm{~m}, 2 \mathrm{H}), 5.96$ (dt, J= $7.7 \mathrm{~Hz}, 1.7 \mathrm{~Hz}, 1 \mathrm{H}), 7.24$ (d, J = 7.7 Hz, 2H, ArH), 7.44 (t, J = 7.7 Hz, 1H, ArH); ${ }^{13} \mathrm{C} \mathrm{NMR}$ $\left(125 \mathrm{MHz}, \mathrm{CD}_{2} \mathrm{Cl}_{2}\right): \delta=22.19$ (2C), 22.24, 22.79 (2C), 23.74 (2C), 24.17 (2C), 24.22 (2C), 
$24.41(2 \mathrm{C}), 27.20$ (2C), 28.48 (2C), 39.01 (t), 63.85 (d), 101.48 (d), 124.53 (2C, d), 128.69 (d), 129.75 (d), 140.29 (s), 146.42 (2C, s), 191.41 (s); IR: (KBr): $v=2927,2862,1697,1633$, $1516,1467,1384,1361,1304,1262,1203,1167,1093,1022,804,760,679,610,546$; HR-

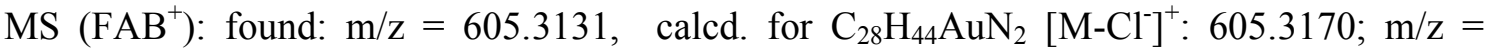
641.2915, calcd. for $\mathrm{C}_{28} \mathrm{H}_{45} \mathrm{AuClN}_{2}[\mathrm{M}+\mathrm{H}]^{+}: 641.2931$.

$17 \mathbf{b}$

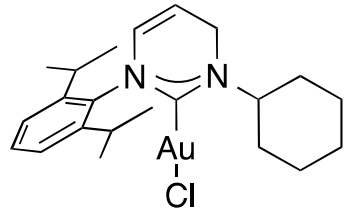

colorless solid; yield: $54.3 \mathrm{mg}, 65 \% ;{ }^{1} \mathrm{H}$ NMR (400 MHz, $\left.\mathrm{CD}_{2} \mathrm{Cl}_{2}\right): \delta=1.10-1.52(\mathrm{~m}, 4 \mathrm{H})$, $1.18(\mathrm{~d}, \mathrm{~J}=7.0 \mathrm{~Hz}, 6 \mathrm{H}), 1.36(\mathrm{~d}, \mathrm{~J}=7.0 \mathrm{~Hz}, 6 \mathrm{H}), 1.63-1.72(\mathrm{~m}, 3 \mathrm{H}), 1.86-1.93(\mathrm{~m}, 3 \mathrm{H}), 3.05$ (sep, J = 7.0 Hz, 2H), 4.02 (s, 2H), 4.77-4.86 (m, 1H), 5.17 (dt, J = 7.9 Hz, 3.2 Hz, 1H), 5.94 $(\mathrm{d}, \mathrm{J}=7.9 \mathrm{~Hz}, 1 \mathrm{H}), 7.24(\mathrm{~d}, \mathrm{~J}=7.7 \mathrm{~Hz}, 2 \mathrm{H}, \mathrm{ArH}), 7.43(\mathrm{t}, \mathrm{J}=7.7 \mathrm{~Hz}, 1 \mathrm{H}, \operatorname{ArH}) ;{ }^{13} \mathrm{C} \mathrm{NMR}$ $\left(100 \mathrm{MHz}, \mathrm{CD}_{2} \mathrm{Cl}_{2}\right): \delta=23.68(2 \mathrm{C}, \mathrm{q}), 24.20(2 \mathrm{C}, \mathrm{q}), 25.22(\mathrm{t}), 25.40(2 \mathrm{C}, \mathrm{t}), 28.41(2 \mathrm{C}, \mathrm{d})$, 29.75 (2C, t), 39.63 (t), 68.63 (d), 101.88 (d), 124.53 (2C, d), 128.40 (d), 129.75 (d), 140.29 (s), $146.43(2 \mathrm{C}, \mathrm{s}), 190.55$ (s); IR (KBr): $v=2925,2855,1681,1512,1463,1385,1360$, 1336, 1319, 1298, 1248, 1184, 1143, 1106, 1056, 1005, 936, 895, 805, 761, 735, 611, 582, $457 \mathrm{~cm}^{-1}$; HR-MS $\left(\mathrm{FAB}^{+}\right)$: found: 558.1869, calcd. for $\mathrm{C}_{22} \mathrm{H}_{32} \mathrm{Au}^{37} \mathrm{ClN}_{2}[\mathrm{M}]^{+}: 521.1890$, found: 556.1887, calcd. for $\mathrm{C}_{22} \mathrm{H}_{32} \mathrm{Au}^{35} \mathrm{ClN}_{2}[\mathrm{M}]^{+}$: 556.1920, found: 521.2186, calcd. for

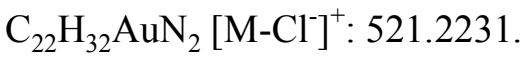

$17 \mathrm{c}$

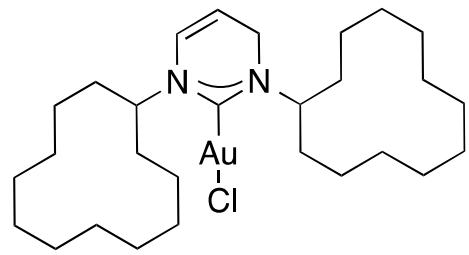

colorless solid; yield: $78.9 \mathrm{mg}, 81 \%,{ }^{1} \mathrm{H}$ NMR (600 MHz, $\left.\mathrm{CD}_{2} \mathrm{Cl}_{2}\right): \delta=1.27-1.44(\mathrm{~m}, 25 \mathrm{H})$, 1.45-1.63 (m, 11H), 1.70-1.84 (m, 8H), $3.74(\mathrm{~d}, \mathrm{~J}=4.1 \mathrm{~Hz}, 2 \mathrm{H}), 5.03(\mathrm{~m}, \mathrm{~J}=6.4 \mathrm{~Hz}, 1 \mathrm{H})$, $5.13(\mathrm{dt}, \mathrm{J}=7.7 \mathrm{~Hz}, 4.1 \mathrm{~Hz}, 1 \mathrm{H}), 5.24(\mathrm{~m}, \mathrm{~J}=6.5 \mathrm{~Hz}, 1 \mathrm{H}), 6.07(\mathrm{~d}, \mathrm{~J}=7.7 \mathrm{~Hz}, 1 \mathrm{H}) ;{ }^{13} \mathrm{C} \mathrm{NMR}$ 
$\left(150 \mathrm{MHz}, \mathrm{CD}_{2} \mathrm{Cl}_{2}\right): \delta=22.17(2 \mathrm{C}, \mathrm{t}), 22.21(2 \mathrm{C}, \mathrm{t}), 22.30(\mathrm{t}), 22.48(\mathrm{t}), 22.95(2 \mathrm{C}, \mathrm{t}), 23.01$ $(2 \mathrm{C}, \mathrm{t}), 24.16(2 \mathrm{C}, \mathrm{t}), 24.23(2 \mathrm{C}, \mathrm{t}), 24.25(2 \mathrm{C}, \mathrm{t}), 24.34(2 \mathrm{C}, \mathrm{t}), 27.52(2 \mathrm{C}, \mathrm{t}), 29.64(2 \mathrm{C}, \mathrm{t})$, 39.23 (t), 62.44 (d), 64.17 (d), 102.83 (d), 124.11 (d), 190.83 (s); $\operatorname{IR}(\mathrm{KBr}): v=2932,2854$, $1631,1504,1467,1444,1410,1355,1308,1282$, 1243, 1204, 1166, 1108, 1046, 902, 717, $599 \mathrm{~cm}^{-1}$; HR-MS $\left(\mathrm{FAB}^{+}\right)$: found: $\mathrm{m} / \mathrm{z}=612.3742$, calcd. for $\mathrm{C}_{28} \mathrm{H}_{51} \mathrm{AuN}_{2}\left[\mathrm{M}-\mathrm{Cl}^{-}\right]^{+}$: 612.3718. 


\section{NMR Spectra}
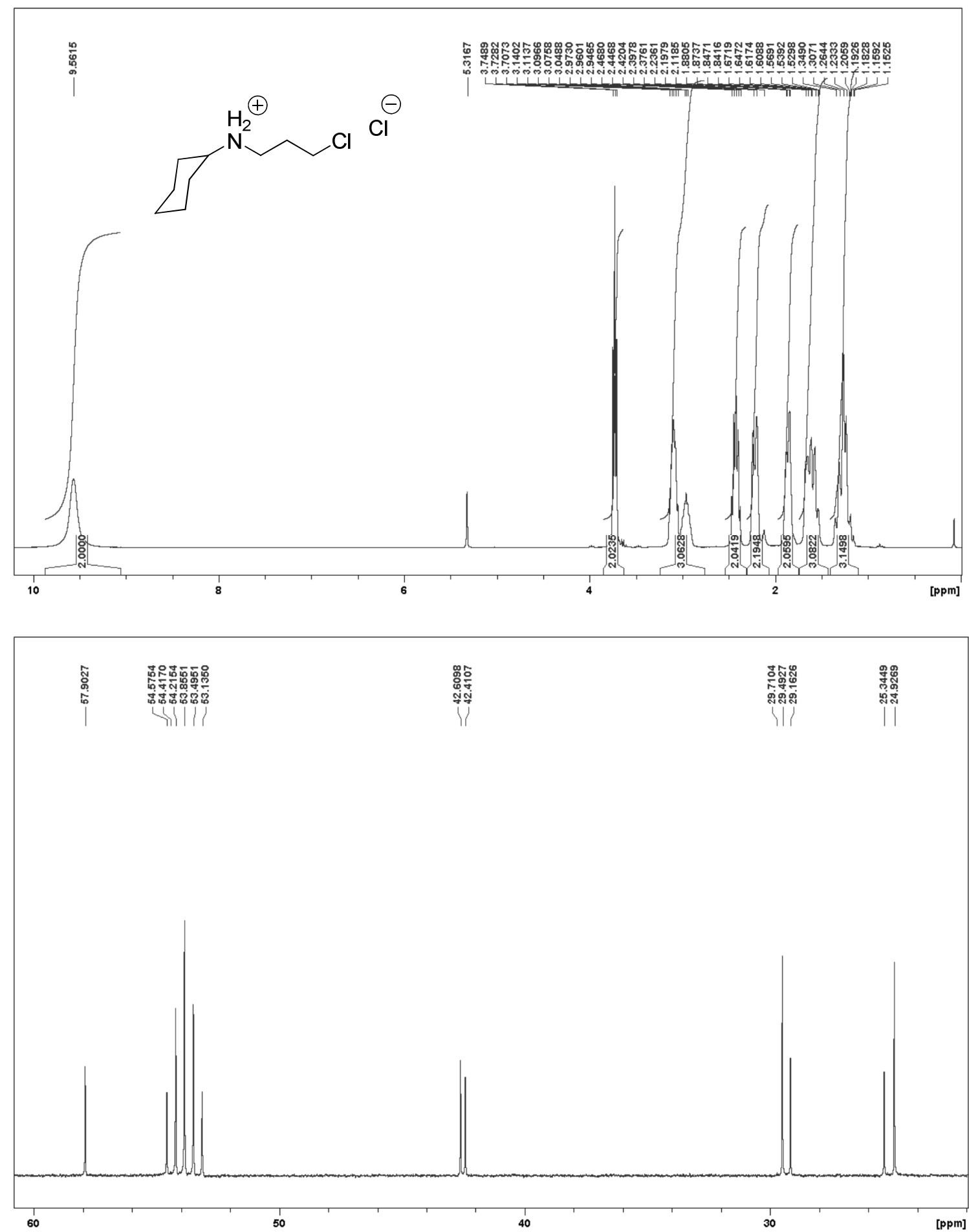

Figure S1: ${ }^{1} \mathrm{H}$ NMR spectrum (top) and ${ }^{13} \mathrm{C}$ NMR spectrum (bottom) of compound 4a 

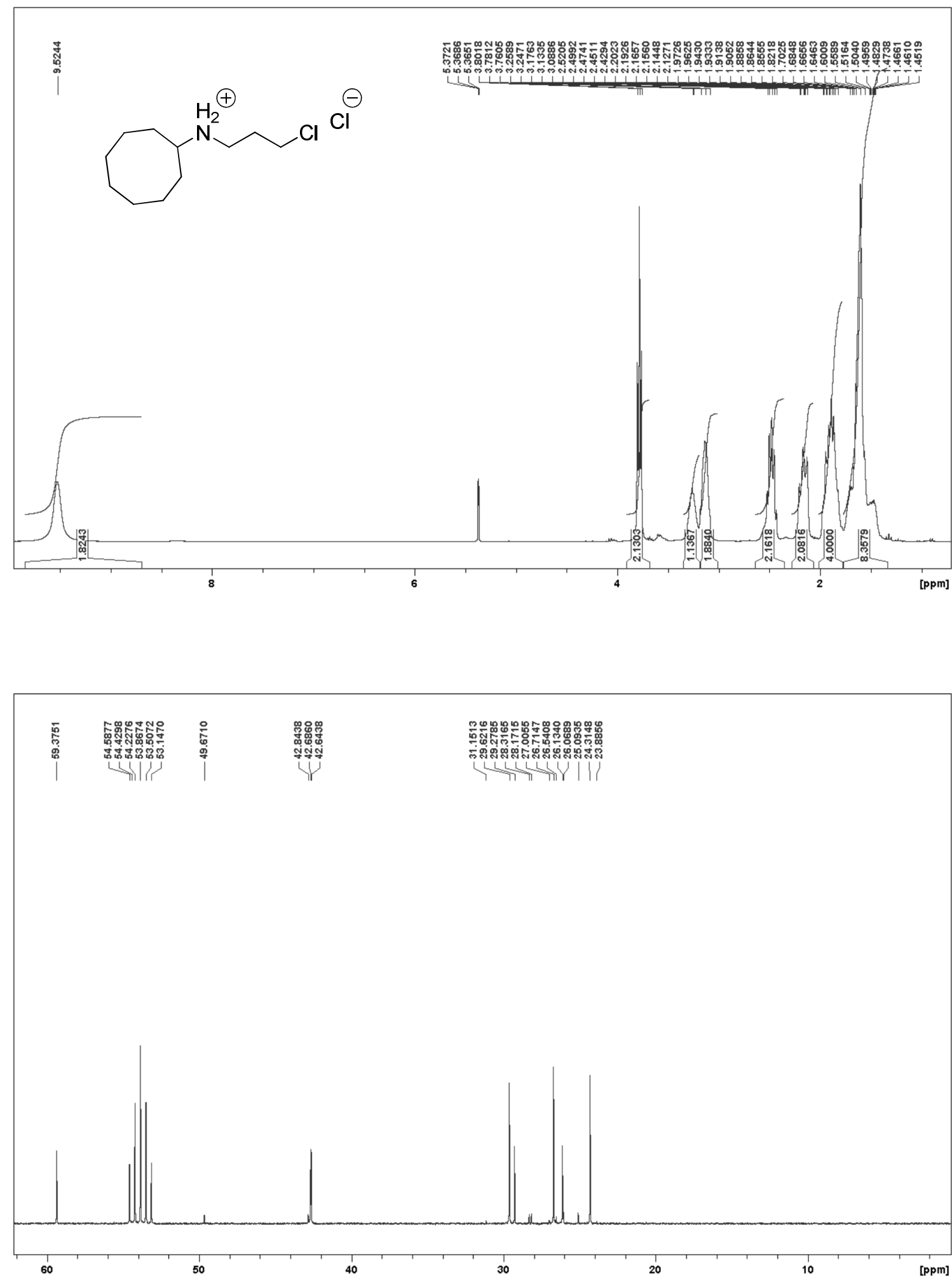

Figure S2: ${ }^{1} \mathrm{H}$ NMR spectrum (top) and ${ }^{13} \mathrm{C}$ NMR spectrum (bottom) of compound $\mathbf{4 b}$ 

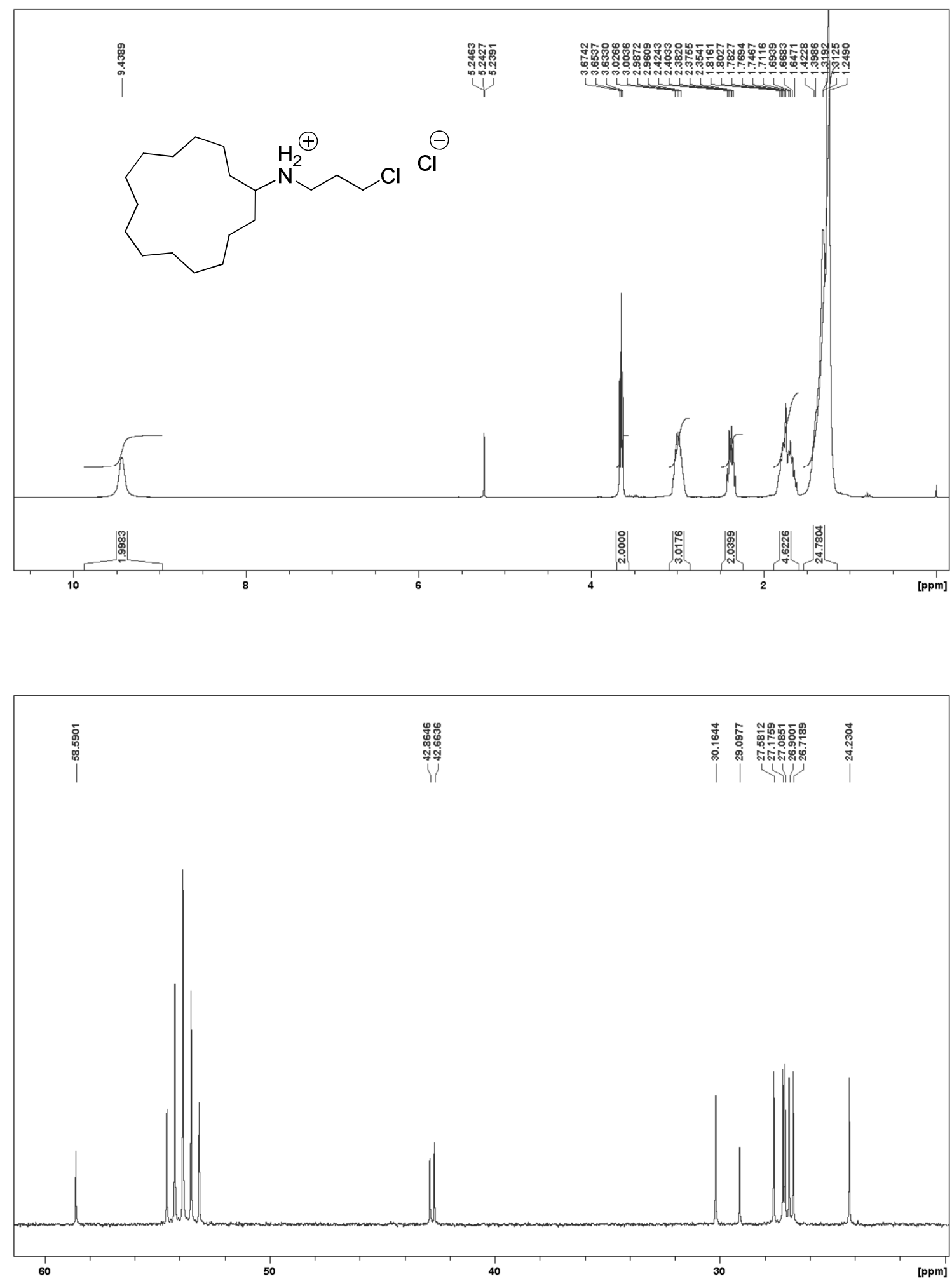

Figure S3: ${ }^{1} \mathrm{H}$ NMR spectrum (top) and ${ }^{13} \mathrm{C}$ NMR spectrum (bottom) of compound 4d 

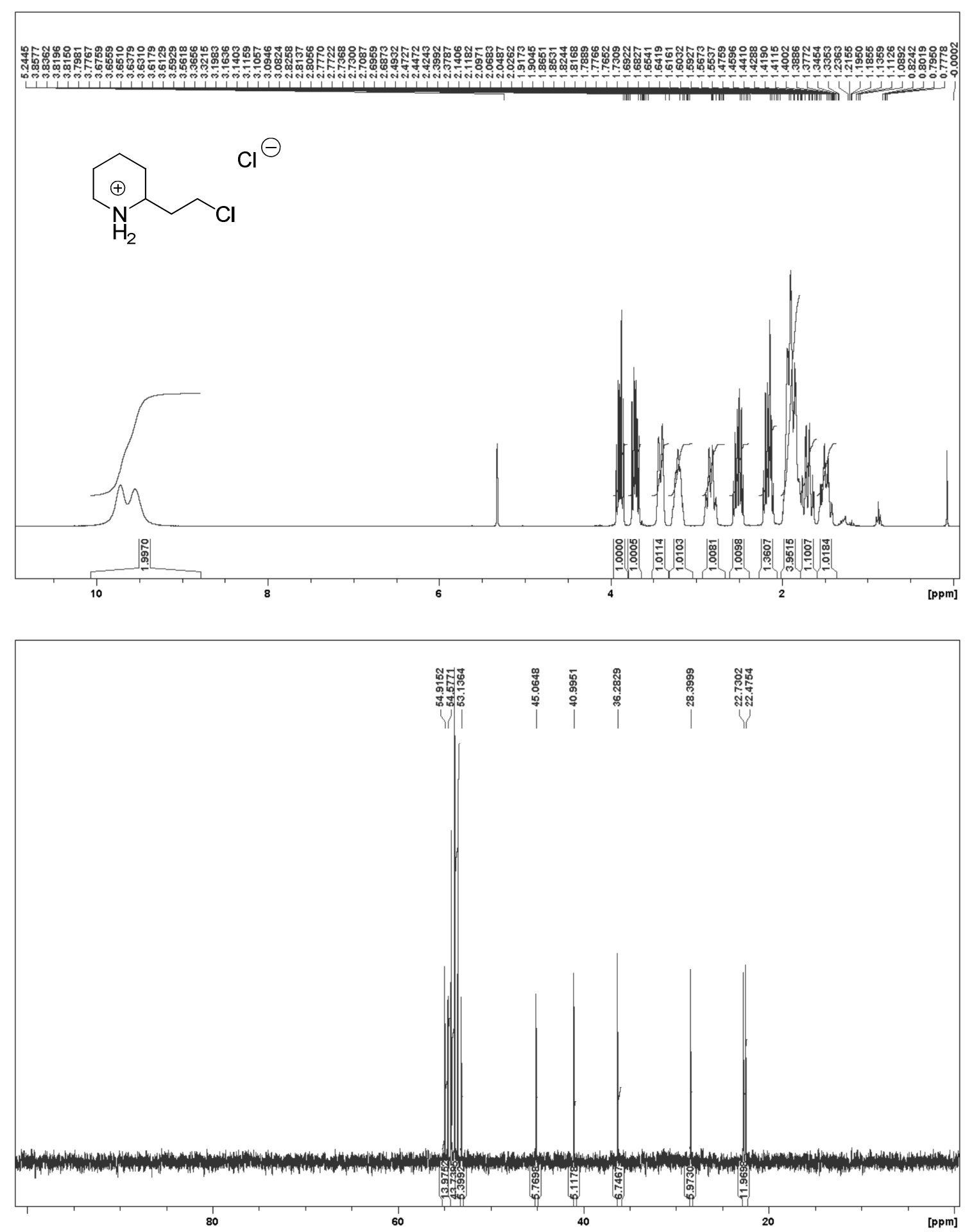

Figure S4: ${ }^{1} \mathrm{H}$ NMR spectrum (top) and ${ }^{13} \mathrm{C}$ NMR spectrum (bottom) of compound $4 \mathrm{e}$ 

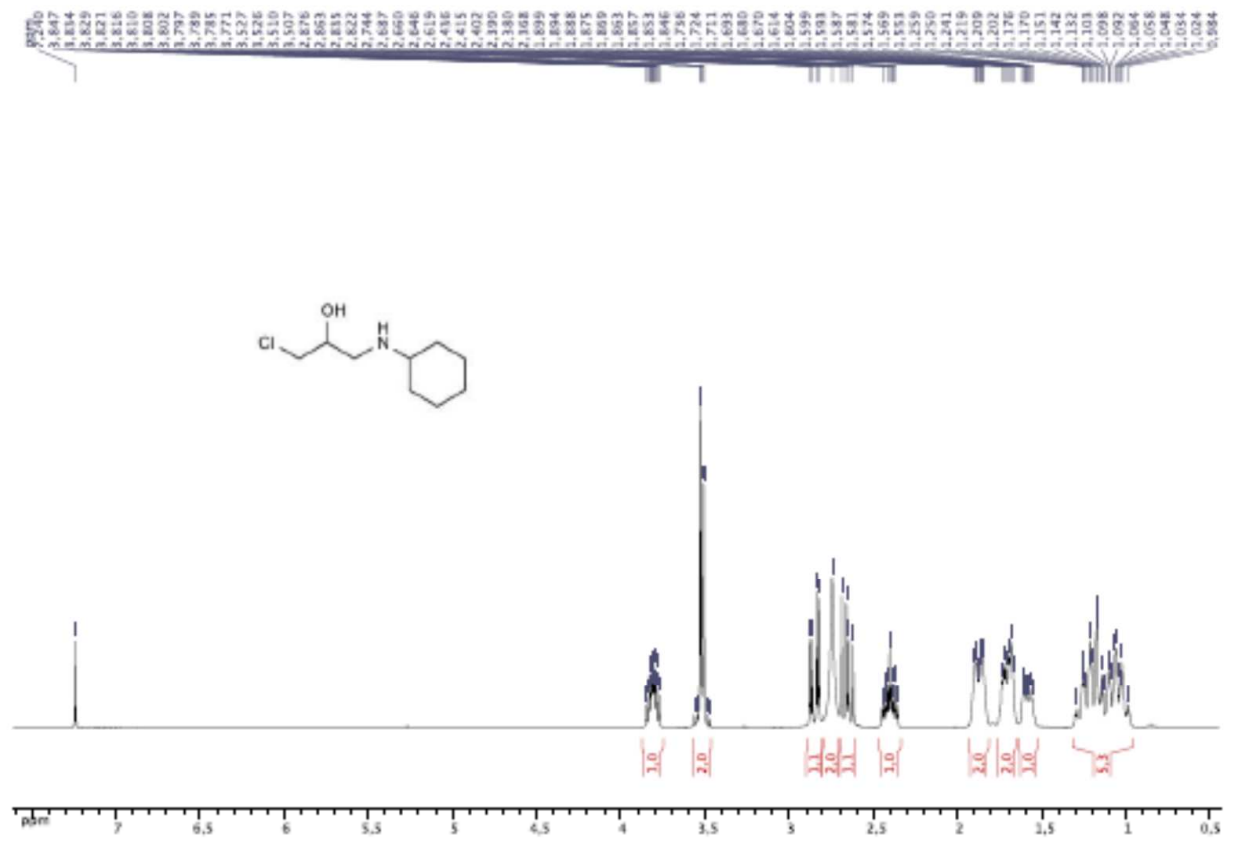

$\frac{1}{2}$
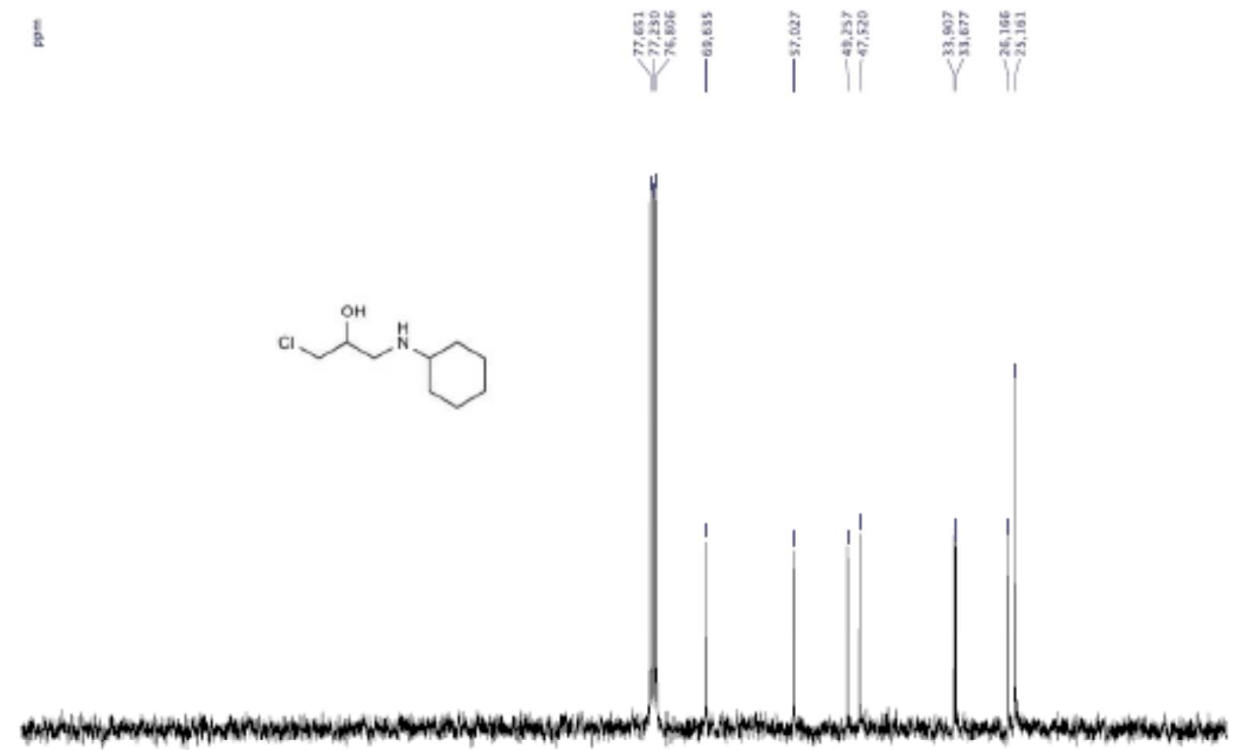

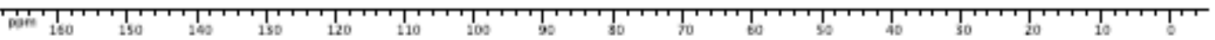

Figure S5: ${ }^{1} \mathrm{H}$ NMR spectrum (top) and ${ }^{13} \mathrm{C}$ NMR spectrum (bottom) of compound $\mathbf{4 f}$ 

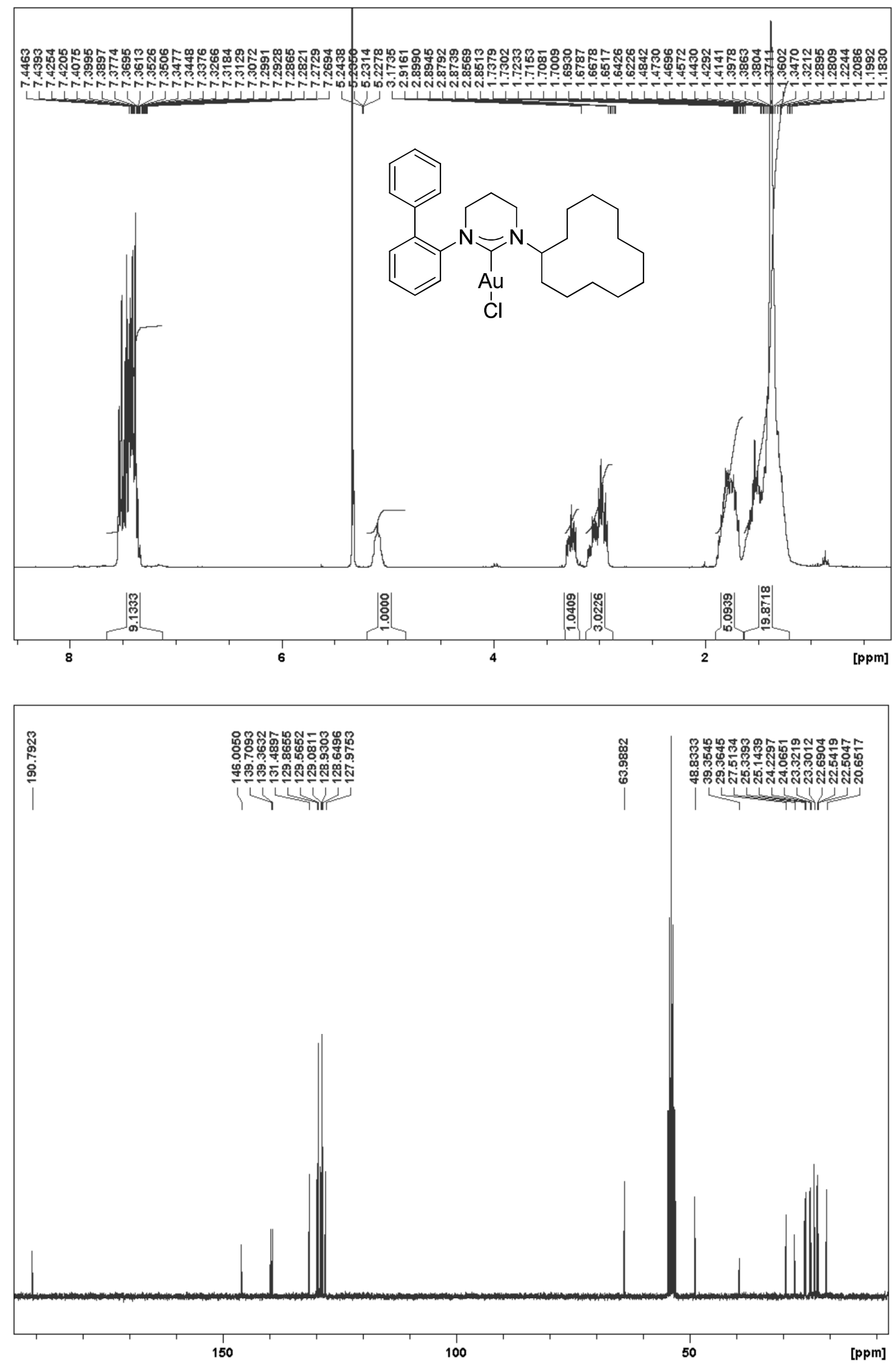

Figure S6: ${ }^{1} \mathrm{H}$ NMR spectrum (top) and ${ }^{13} \mathrm{C}$ NMR spectrum (bottom) of compound 6a 

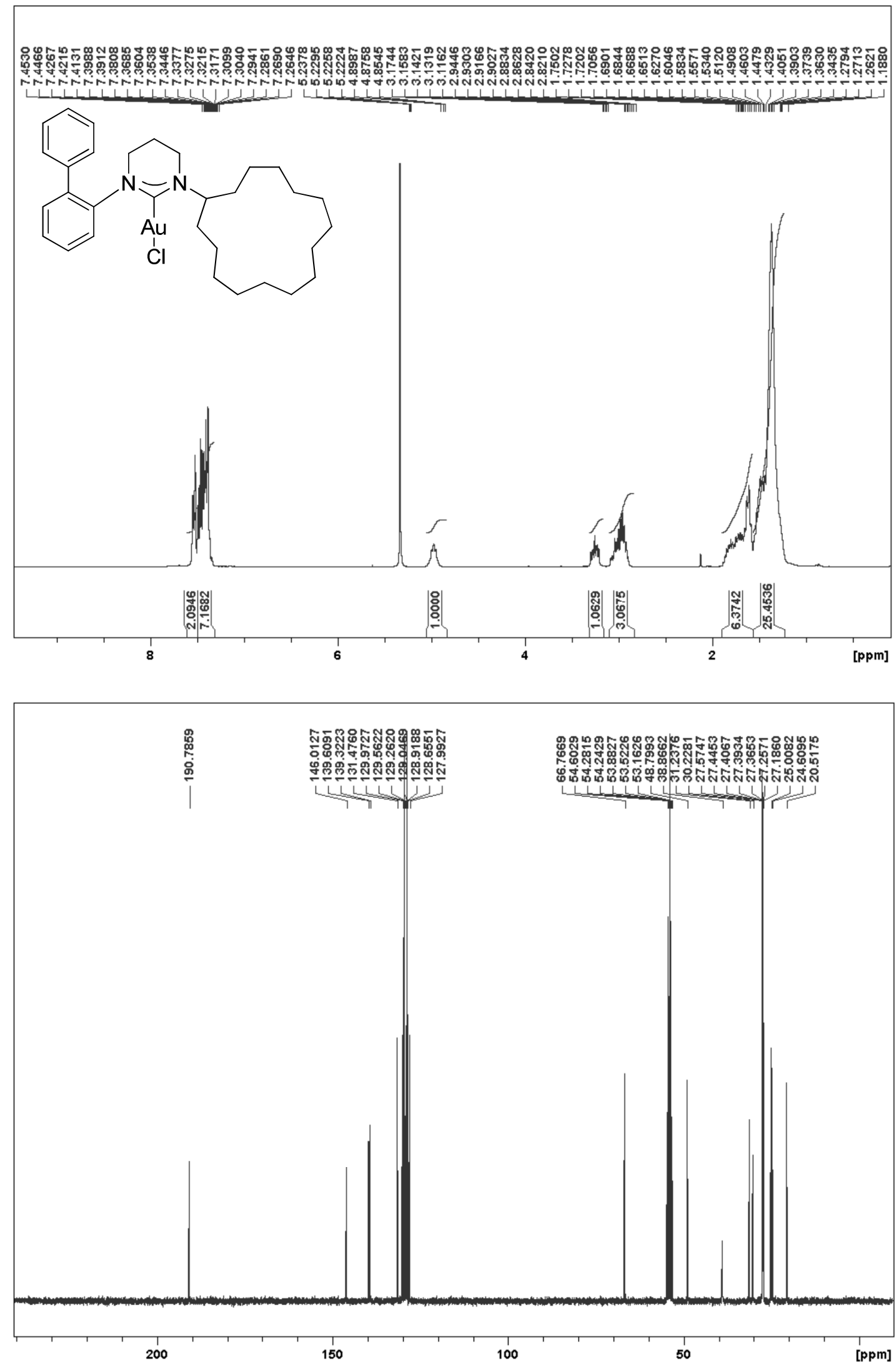

Figure S7: ${ }^{1} \mathrm{H}$ NMR spectrum (top) and ${ }^{13} \mathrm{C}$ NMR spectrum (bottom) of compound $\mathbf{6 b}$ 

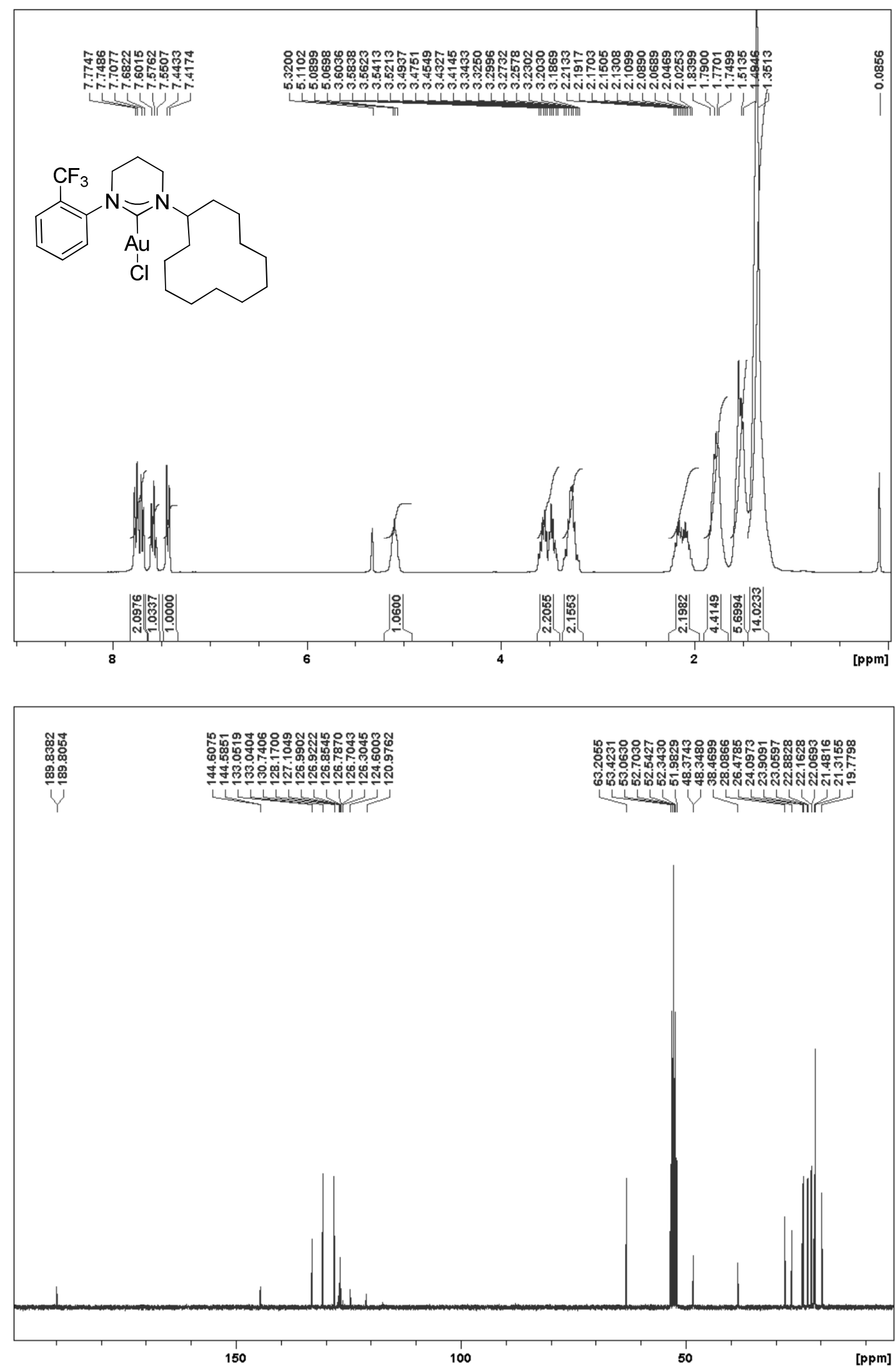

Figure S8: ${ }^{1} \mathrm{H}$ NMR spectrum (top) and ${ }^{13} \mathrm{C}$ NMR spectrum (bottom) of compound $\mathbf{6 c}$ 

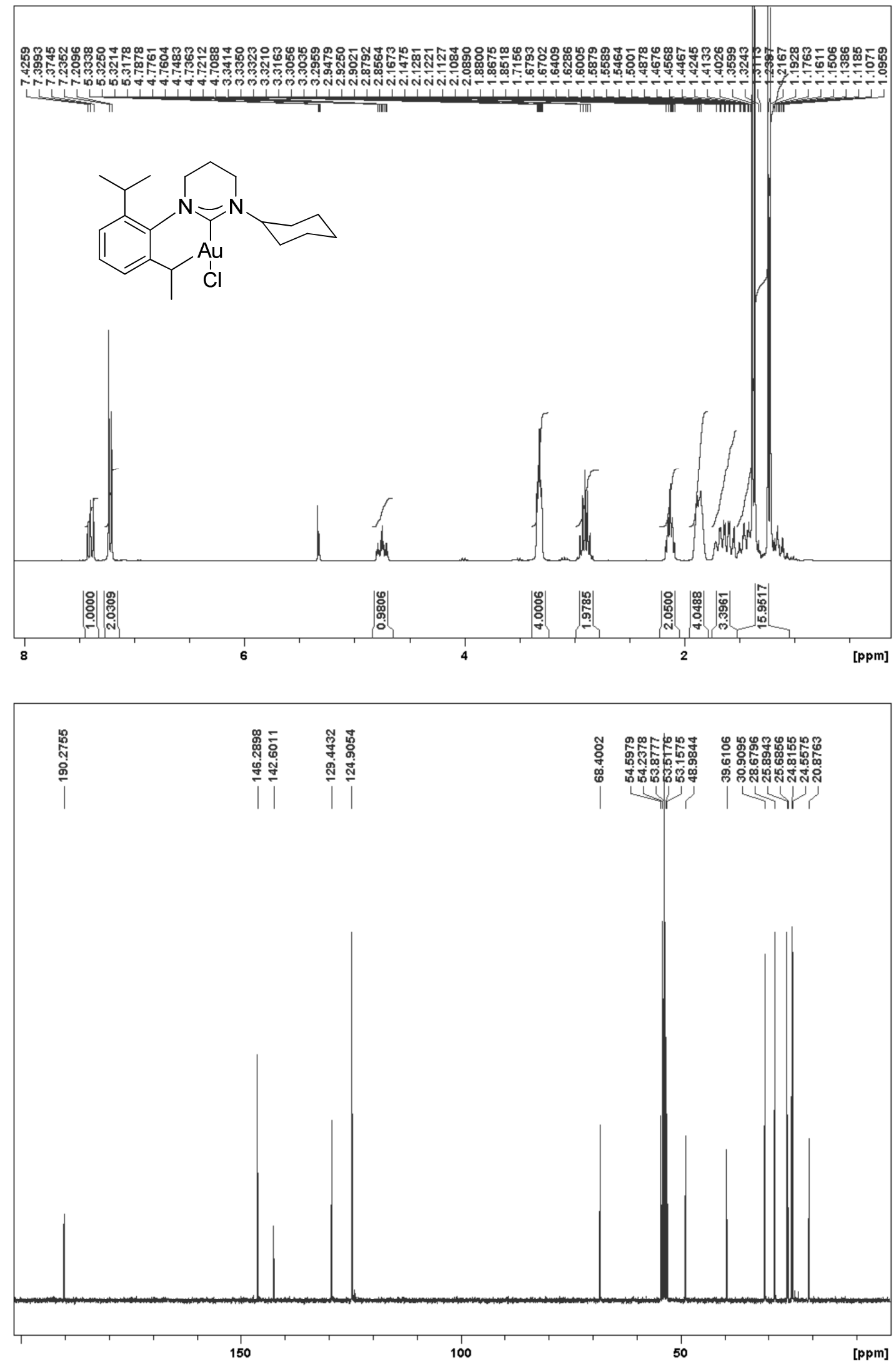

Figure S9: ${ }^{1} \mathrm{H}$ NMR spectrum (top) and ${ }^{13} \mathrm{C}$ NMR spectrum (bottom) of compound 6d 

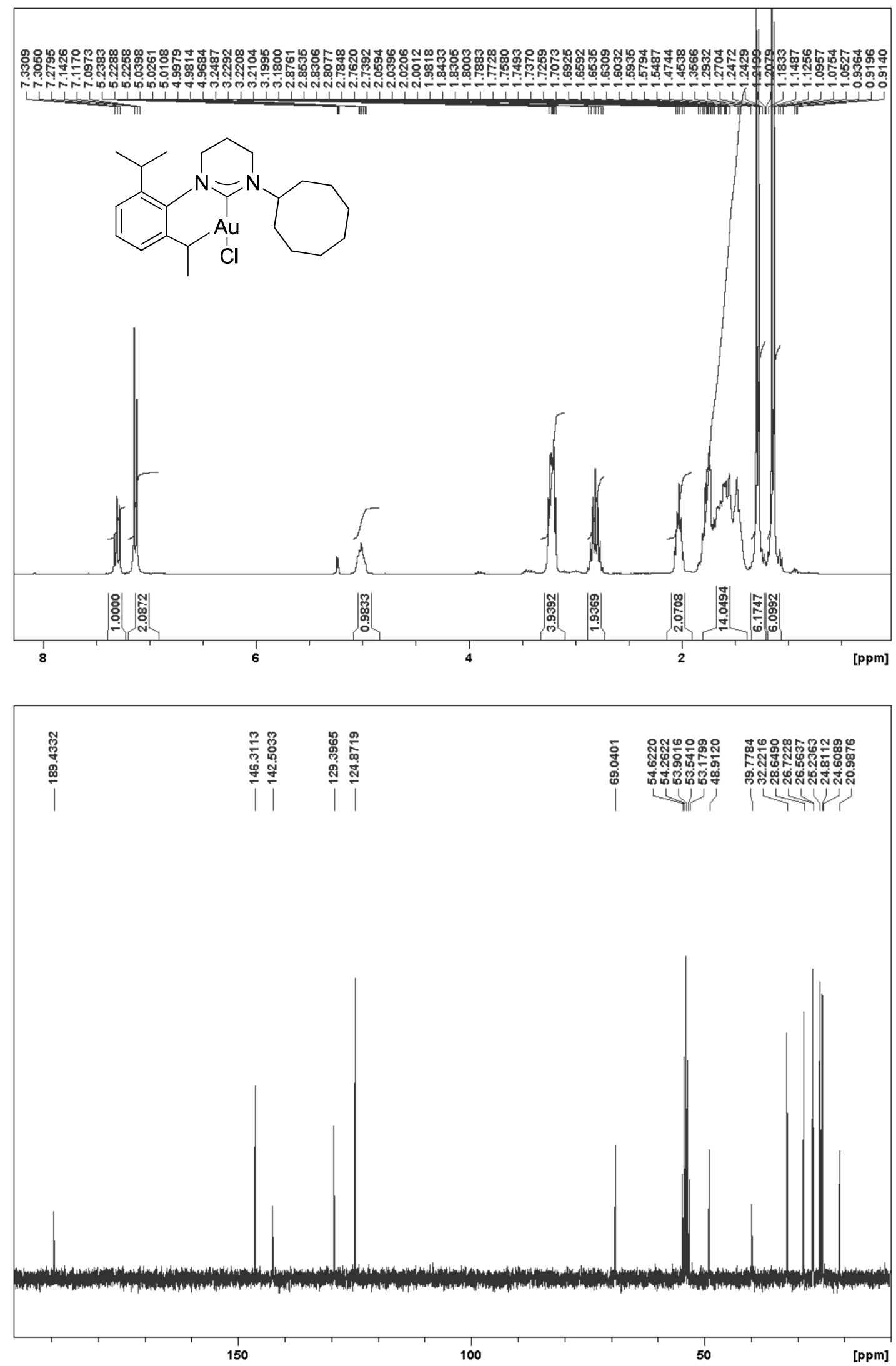

Figure S10: ${ }^{1} \mathrm{H}$ NMR spectrum (top) and ${ }^{13} \mathrm{C}$ NMR spectrum (bottom) of compound $6 \mathbf{e}$ 

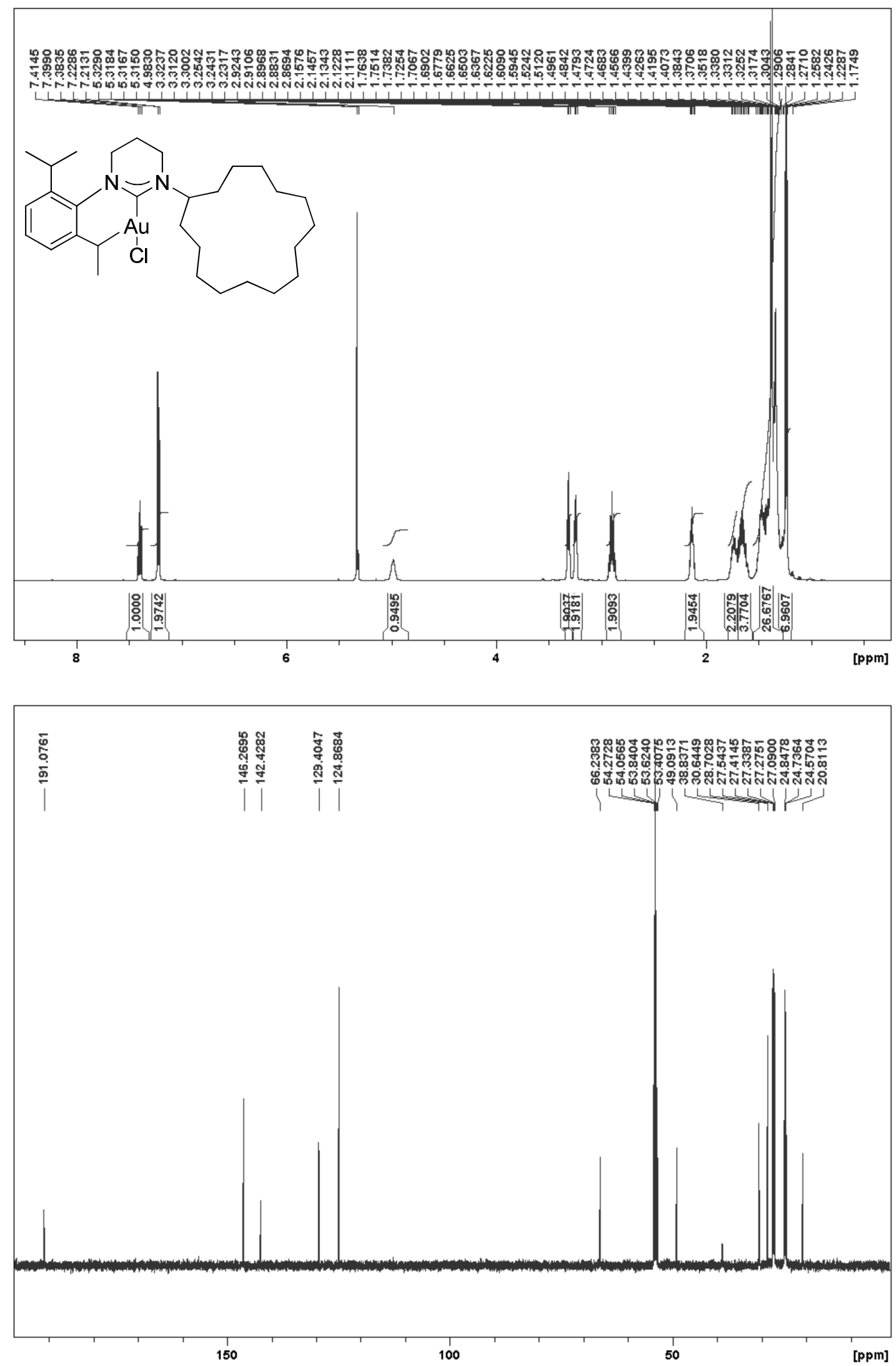

Figure S11: ${ }^{1} \mathrm{H}$ NMR spectrum (top) and ${ }^{13} \mathrm{C}$ NMR spectrum (bottom) of compound $6 \mathbf{f}$ 

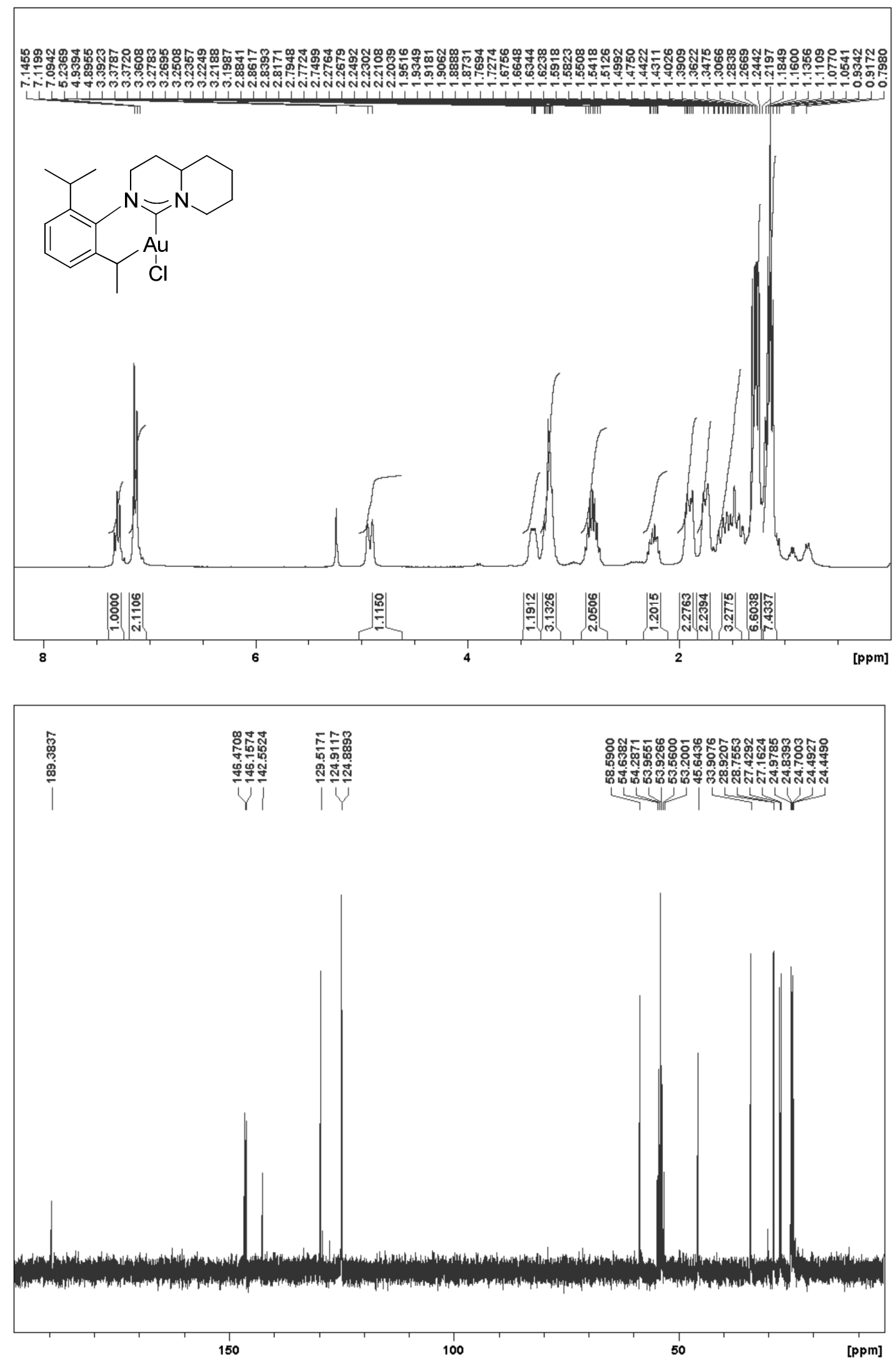

Figure S12: ${ }^{1} \mathrm{H}$ NMR spectrum (top) and ${ }^{13} \mathrm{C}$ NMR spectrum (bottom) of compound $\mathbf{6 g}$ 

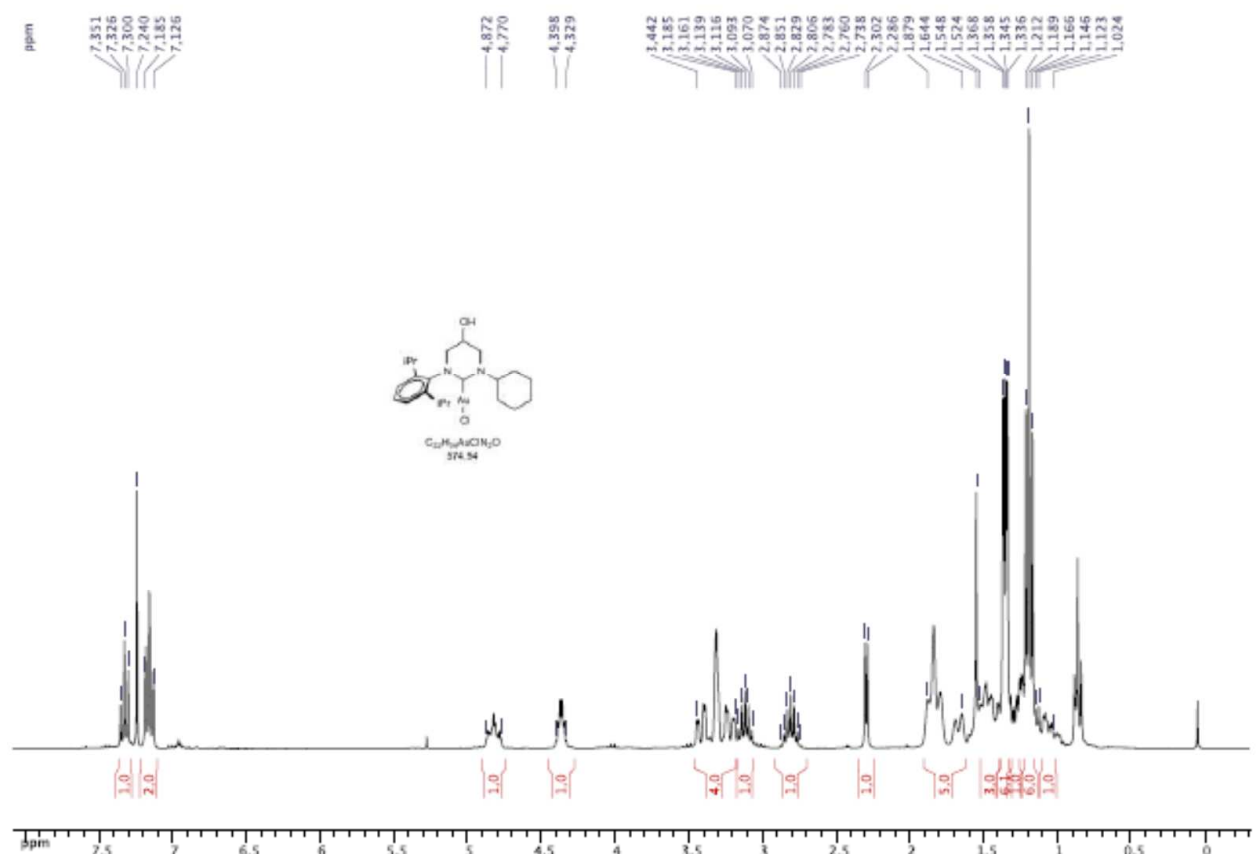

등
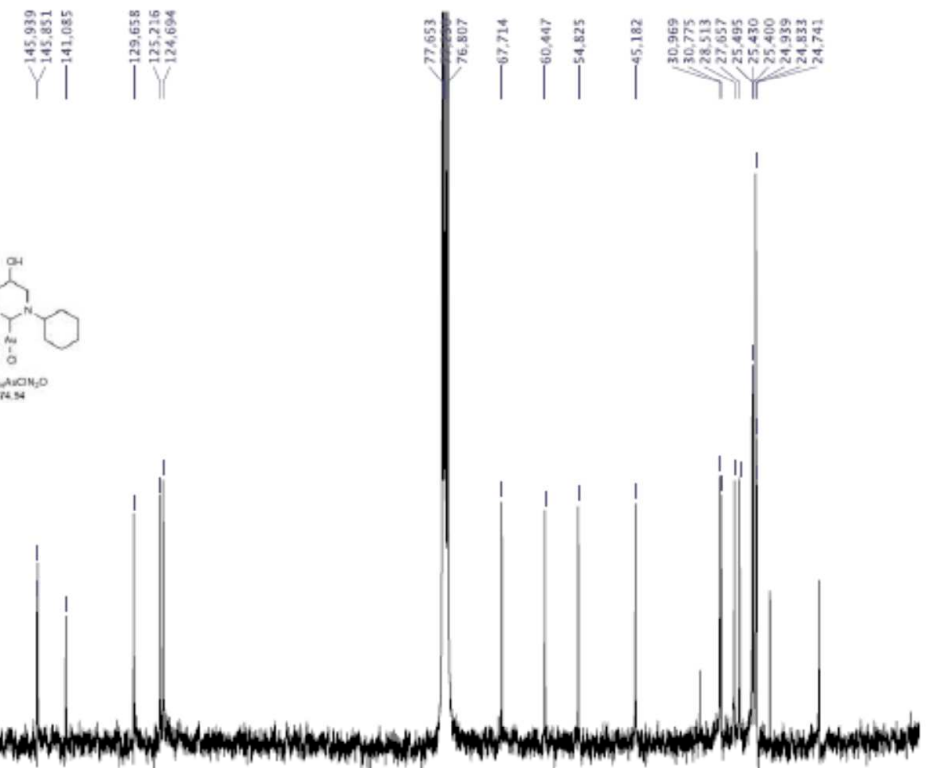

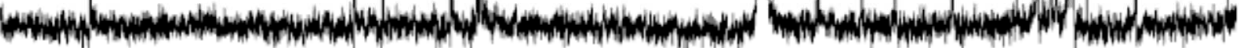

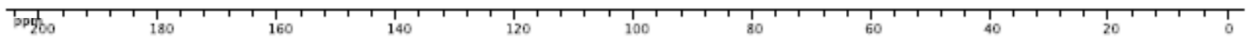

Figure S13: ${ }^{1} \mathrm{H}$ NMR spectrum (top) and ${ }^{13} \mathrm{C}$ NMR spectrum (bottom) of compound $\mathbf{6 h}$ 


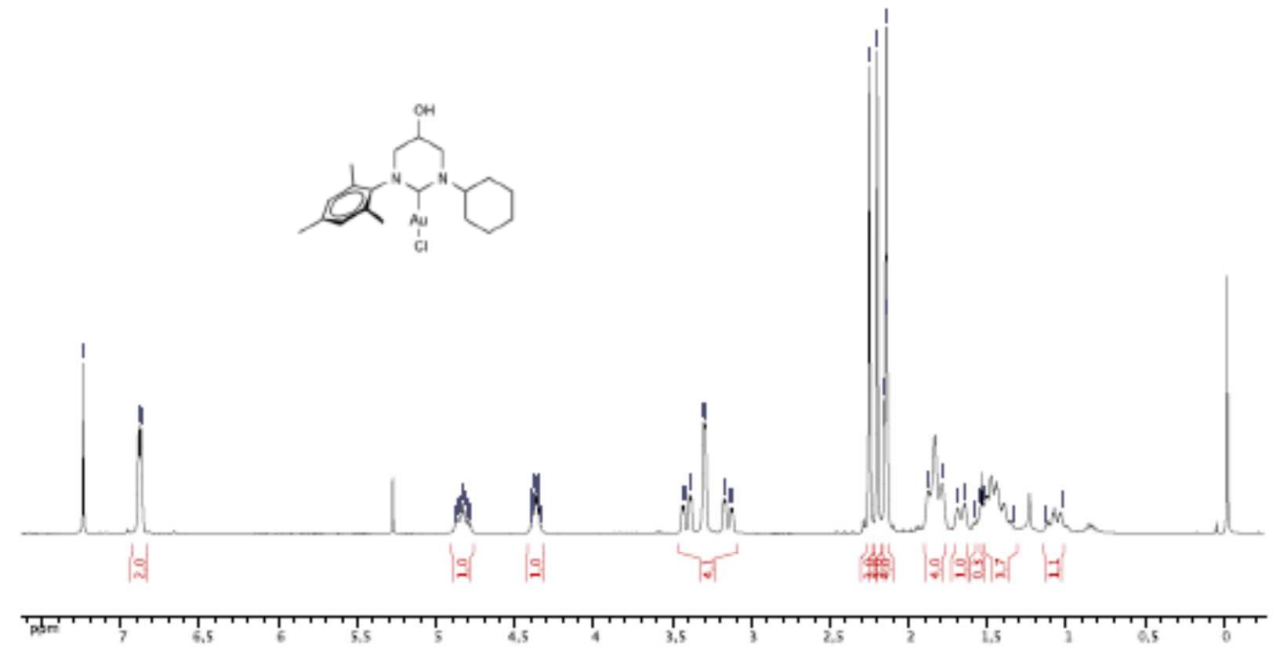

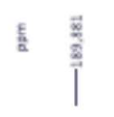
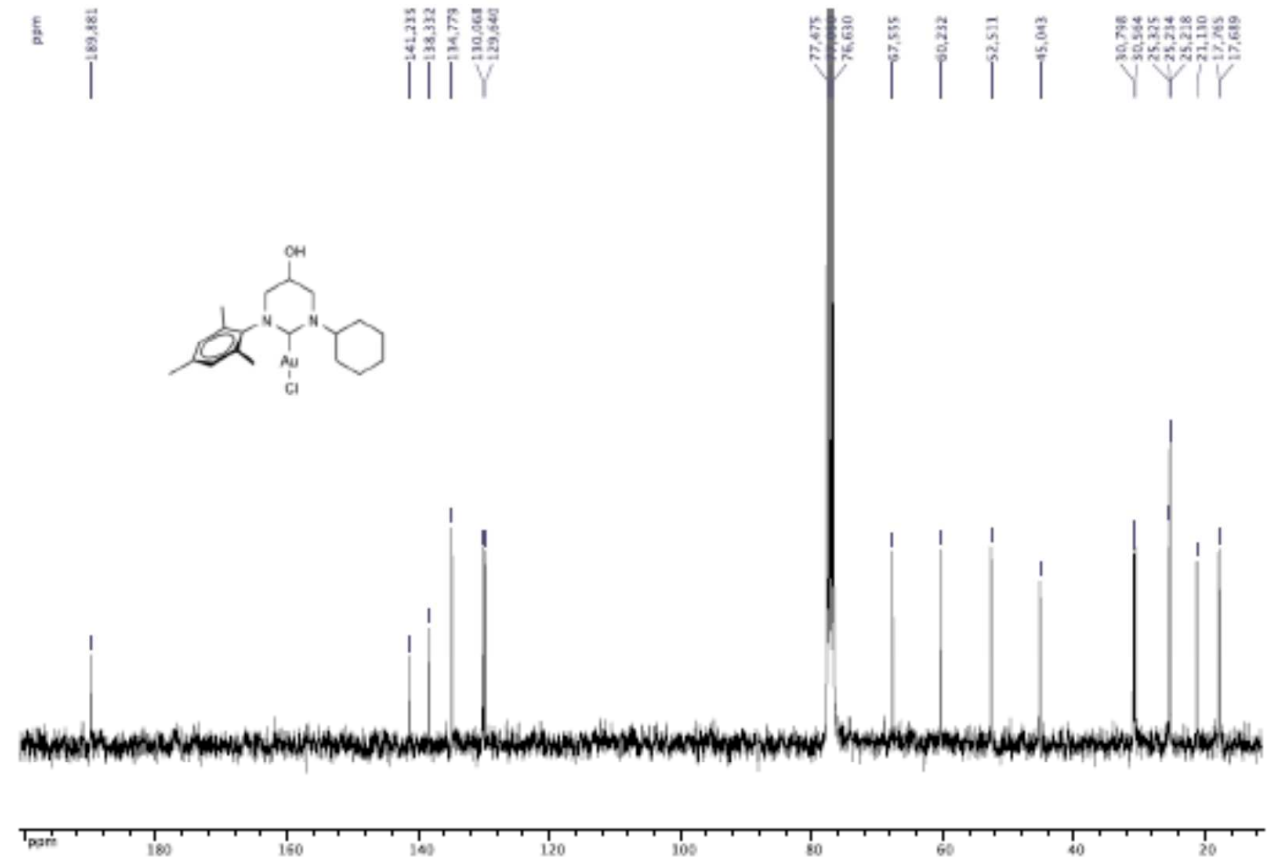

Figure S14: ${ }^{1} \mathrm{H}$ NMR spectrum (top) and ${ }^{13} \mathrm{C}$ NMR spectrum (bottom) of compound $6 \mathbf{i}$ 

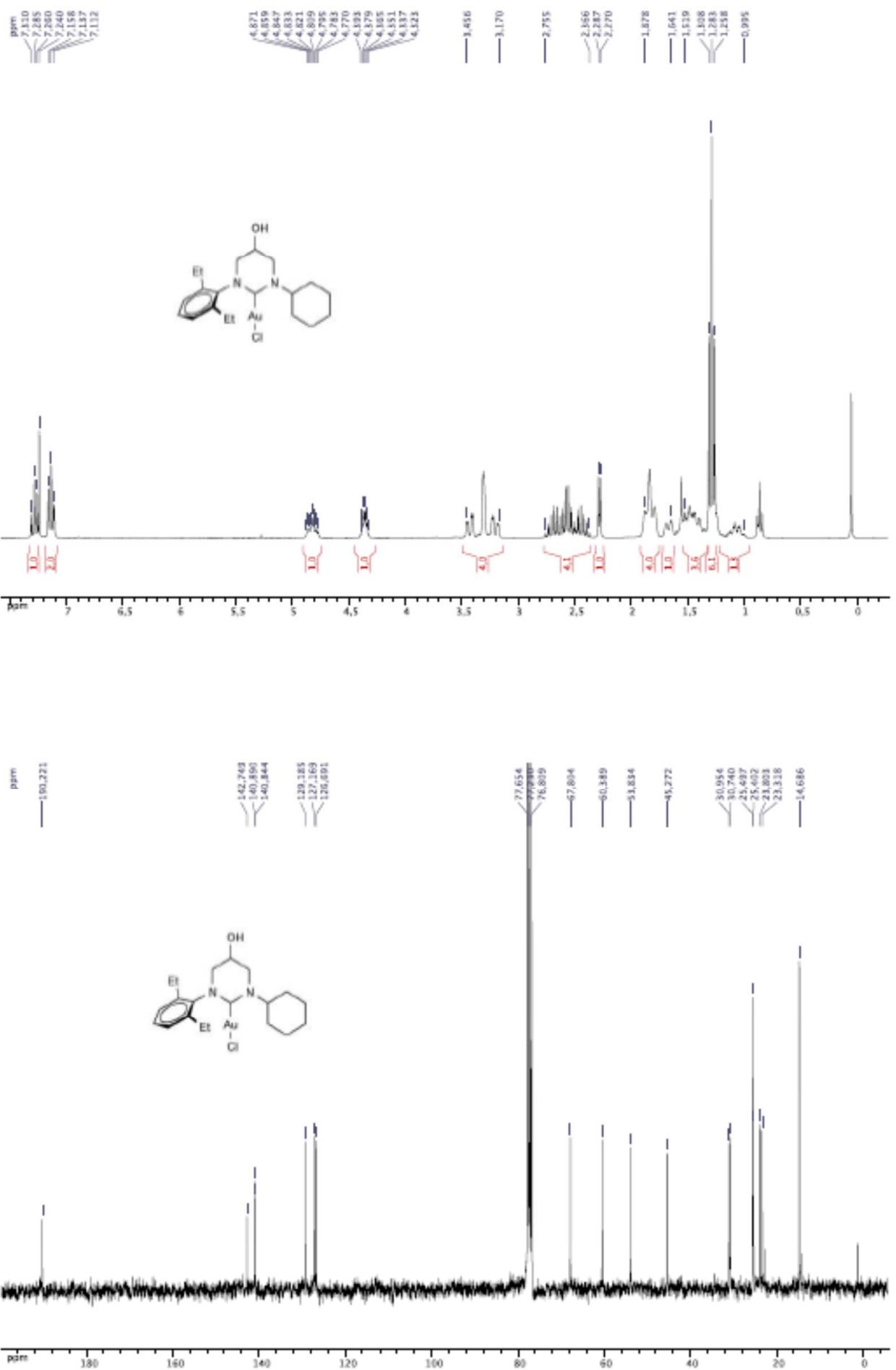

Figure S15: ${ }^{1} \mathrm{H}$ NMR spectrum (top) and ${ }^{13} \mathrm{C}$ NMR spectrum (bottom) of compound $\mathbf{6 j}$ 

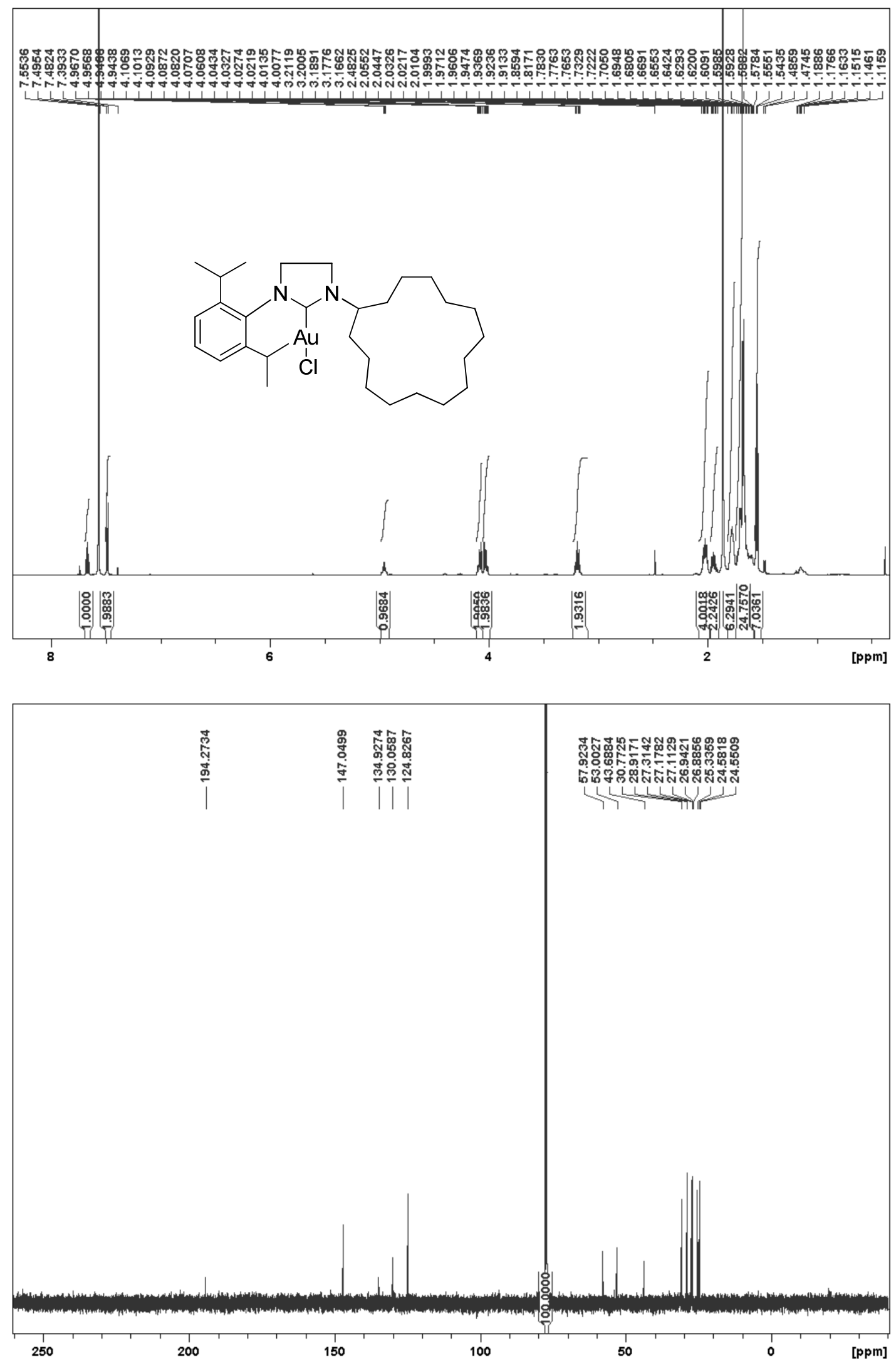

Figure S16: ${ }^{1} \mathrm{H}$ NMR spectrum (top) and ${ }^{13} \mathrm{C}$ NMR spectrum (bottom) of compound 8 

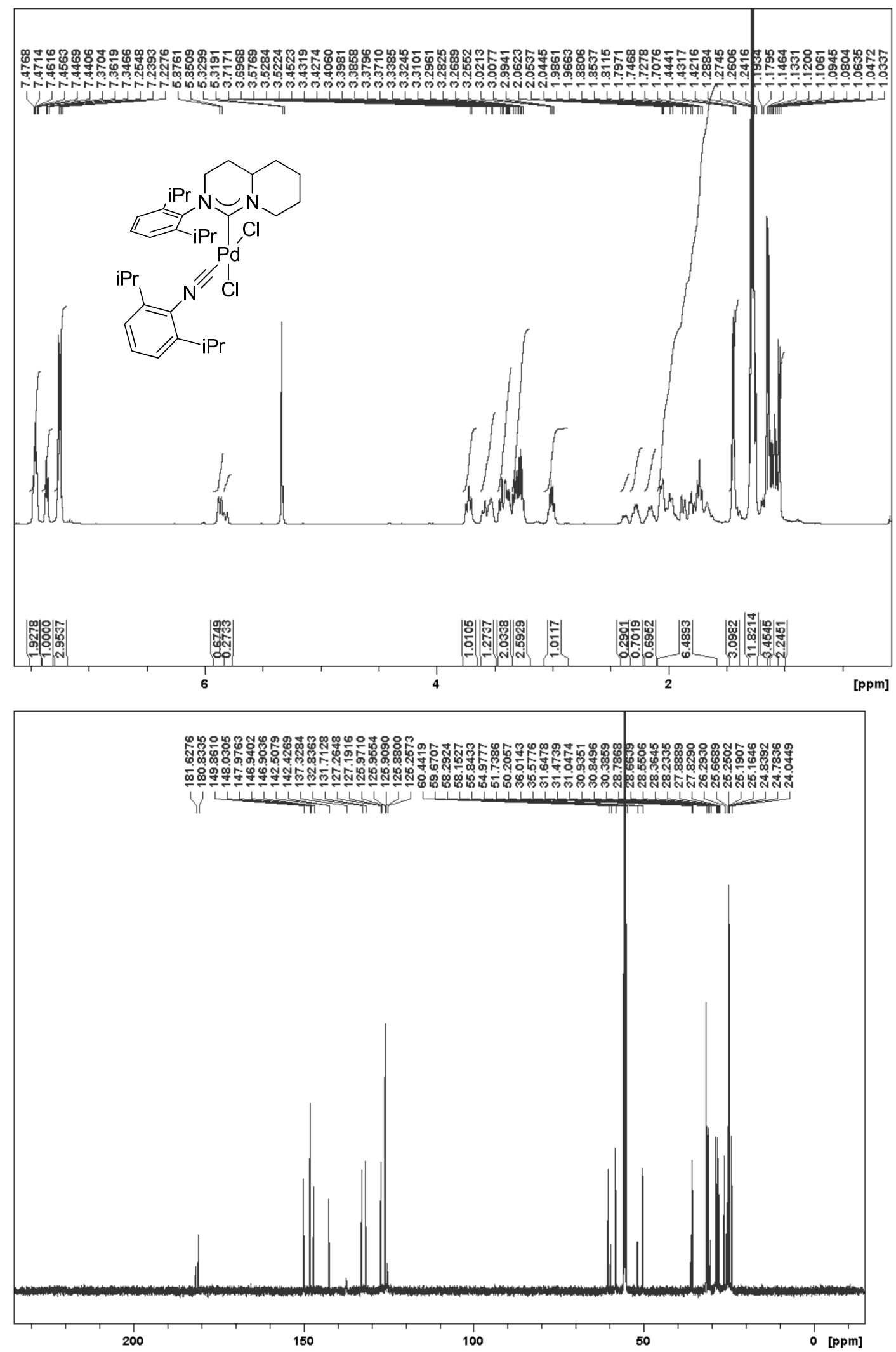

Figure S17: ${ }^{1} \mathrm{H}$ NMR spectrum (top) and ${ }^{13} \mathrm{C}$ NMR spectrum (bottom) of compound $\mathbf{1 0}$ 

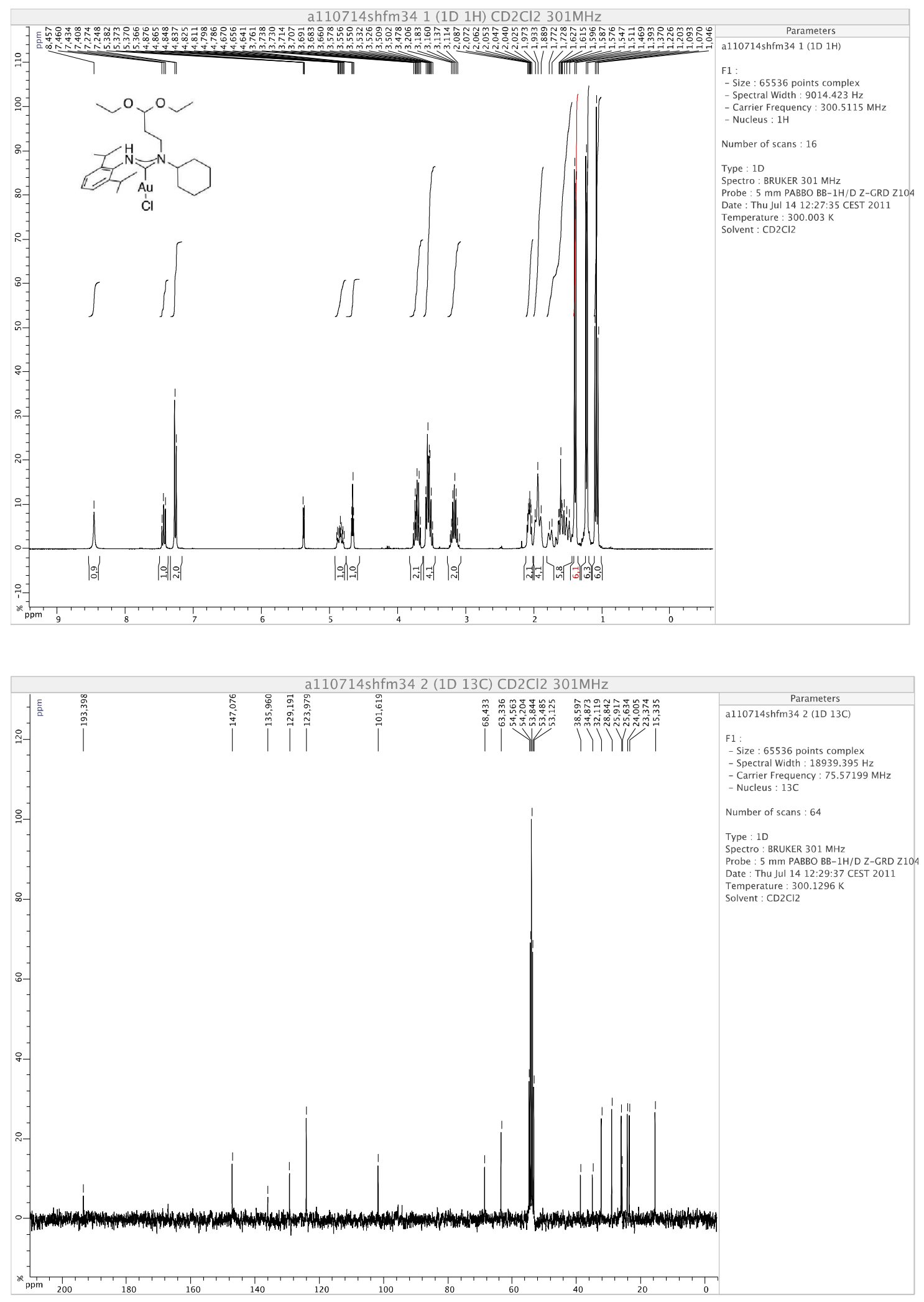

Figure S18: ${ }^{1} \mathrm{H}$ NMR spectrum (top) and ${ }^{13} \mathrm{C}$ NMR spectrum (bottom) of compound 15d 

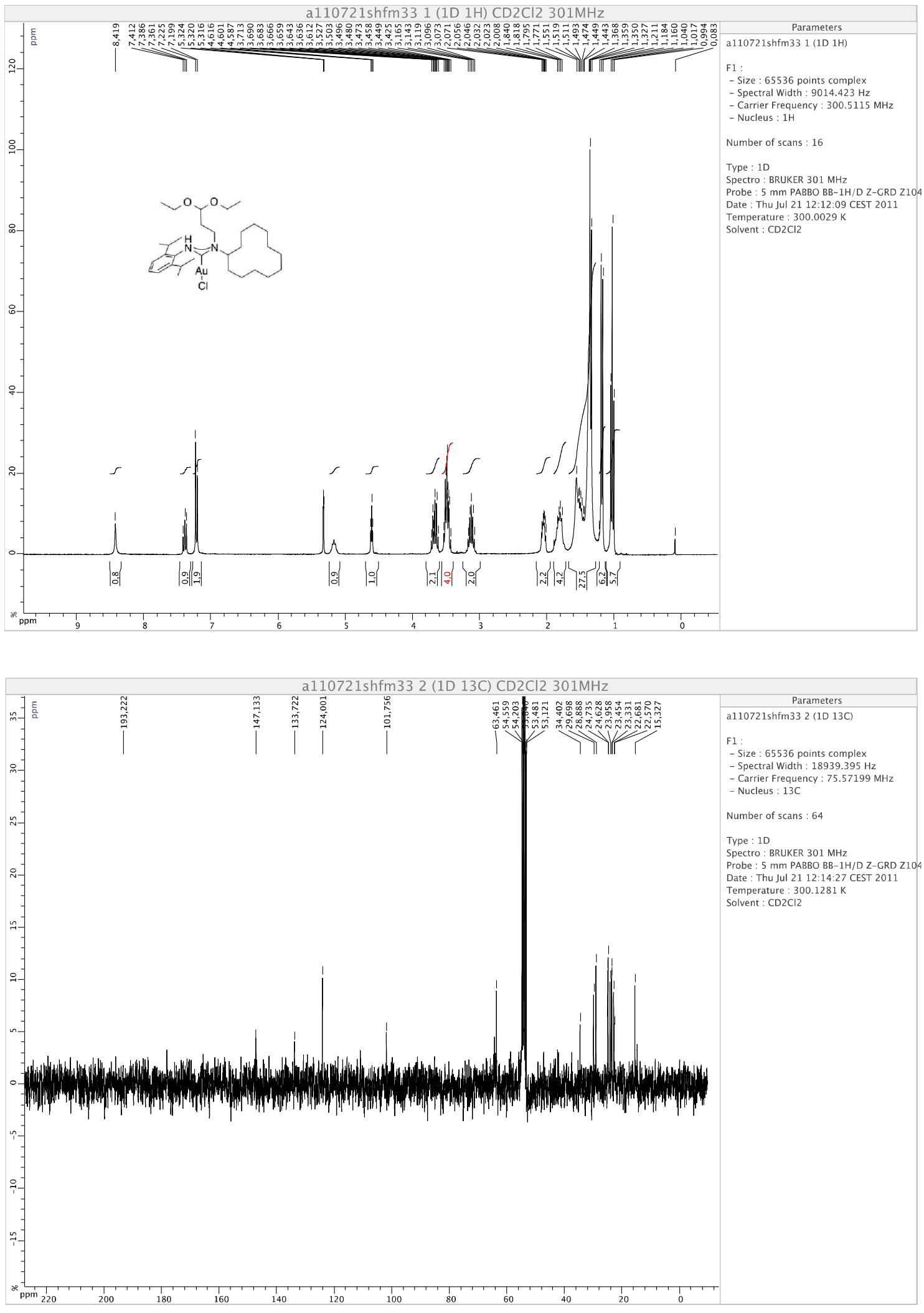

Figure S19: ${ }^{1} \mathrm{H}$ NMR spectrum (top) and ${ }^{13} \mathrm{C}$ NMR spectrum (bottom) of compound 15a 

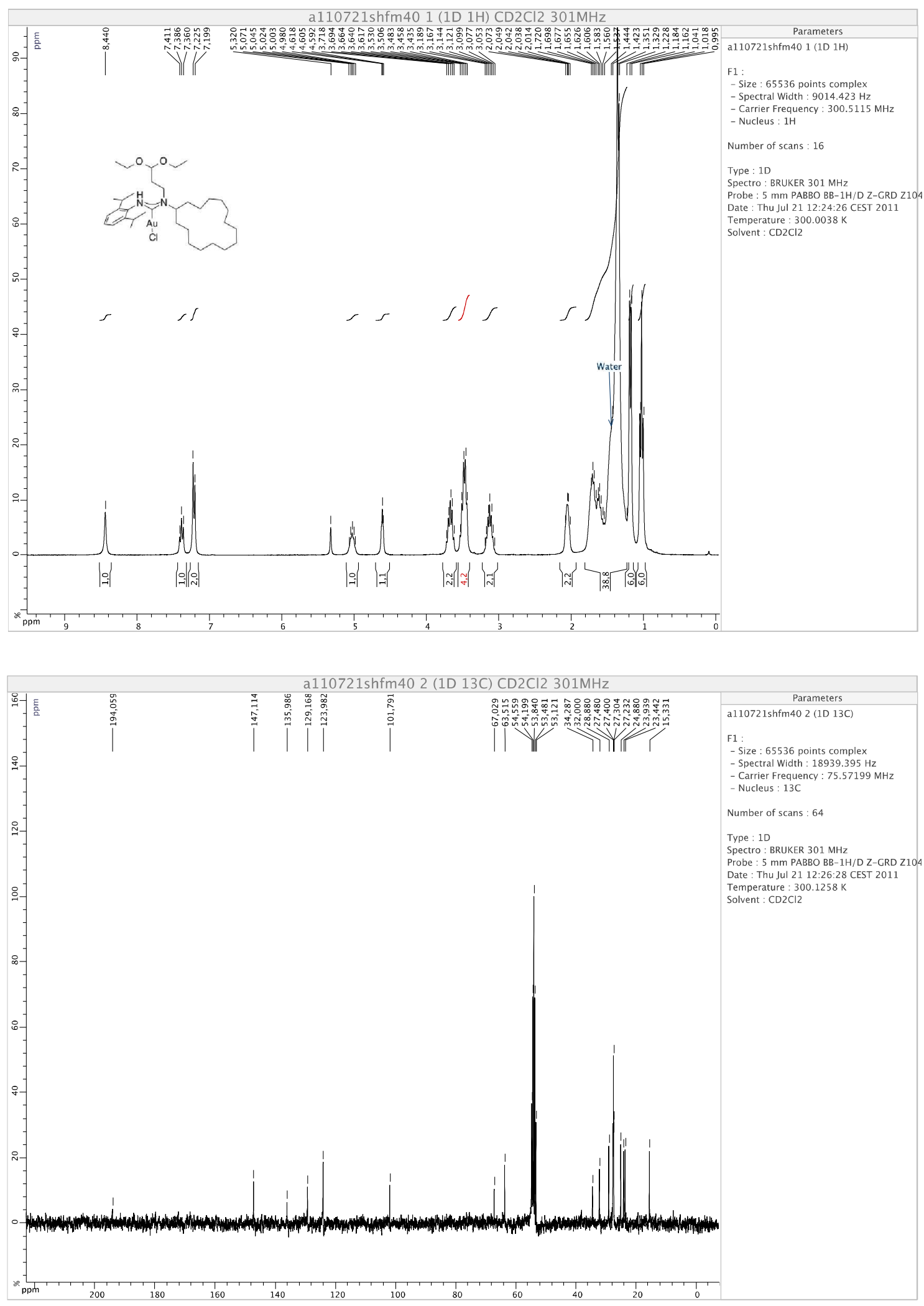

Figure S20: ${ }^{1} \mathrm{H}$ NMR spectrum (top) and ${ }^{13} \mathrm{C}$ NMR spectrum (bottom) of compound $15 \mathrm{c}$ 

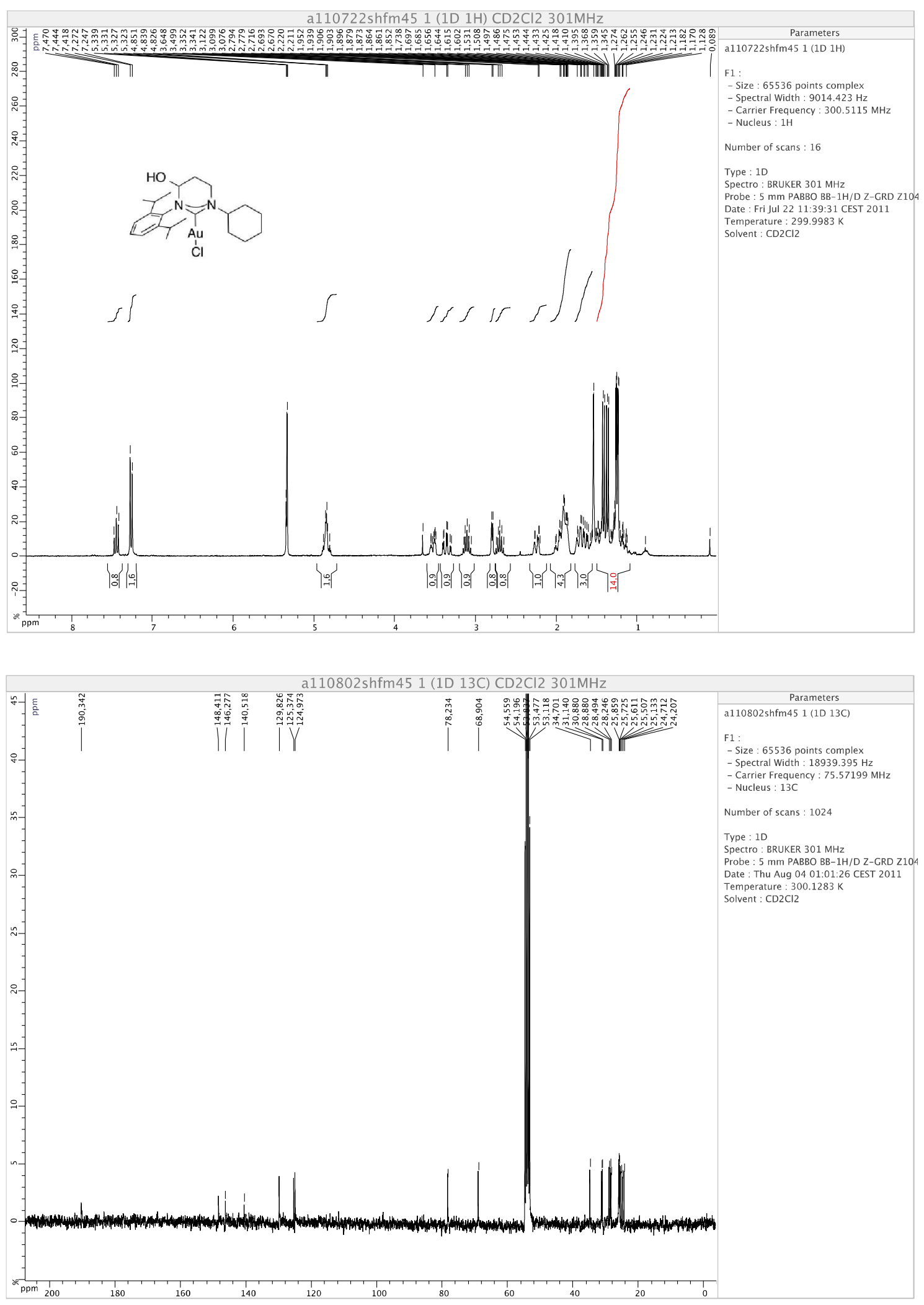

Figure S21: ${ }^{1} \mathrm{H}$ NMR spectrum (top) and ${ }^{13} \mathrm{C}$ NMR spectrum (bottom) of compound 16b 

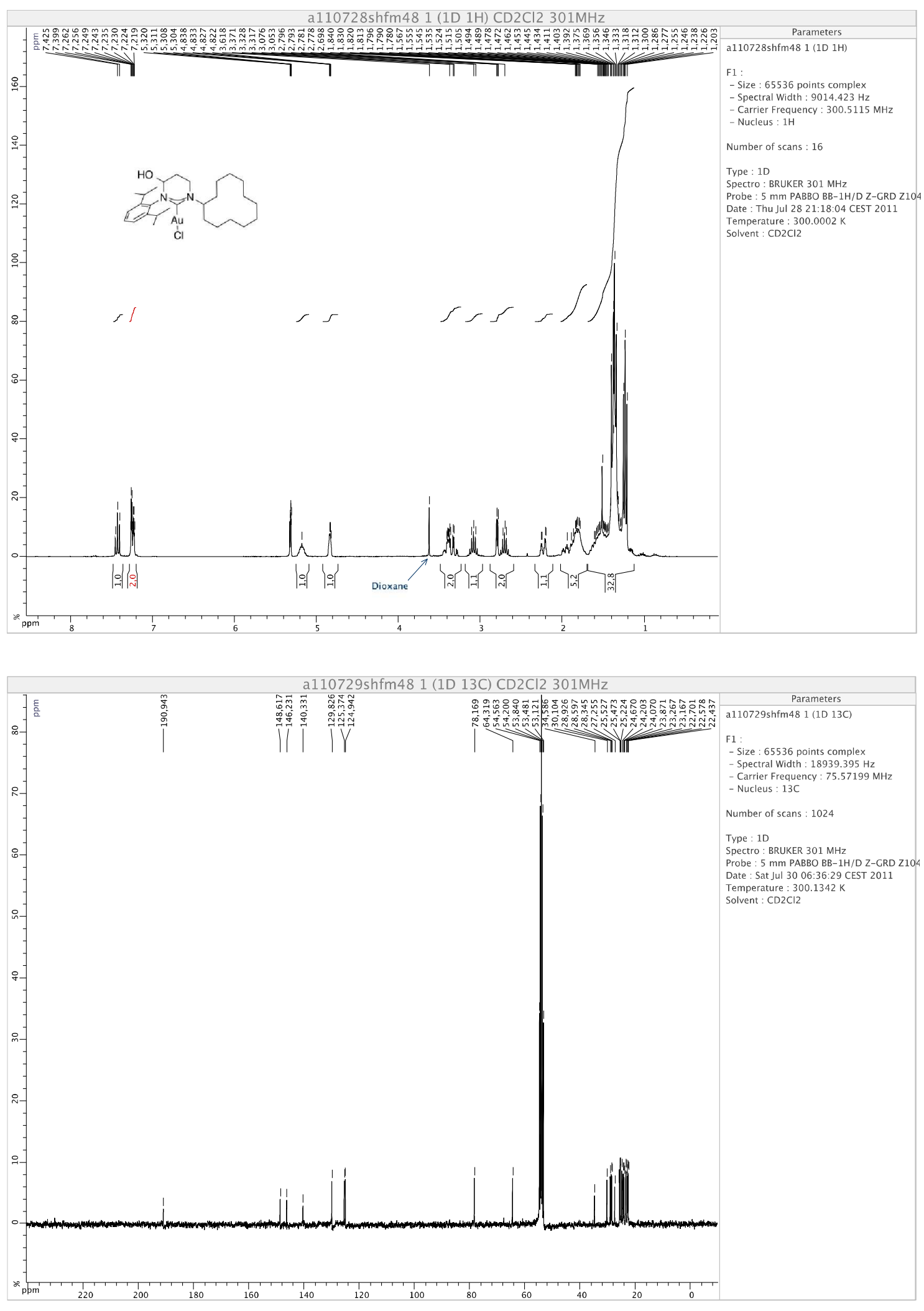

Figure S22: ${ }^{1} \mathrm{H}$ NMR spectrum (top) and ${ }^{13} \mathrm{C}$ NMR spectrum (bottom) of compound 16a 

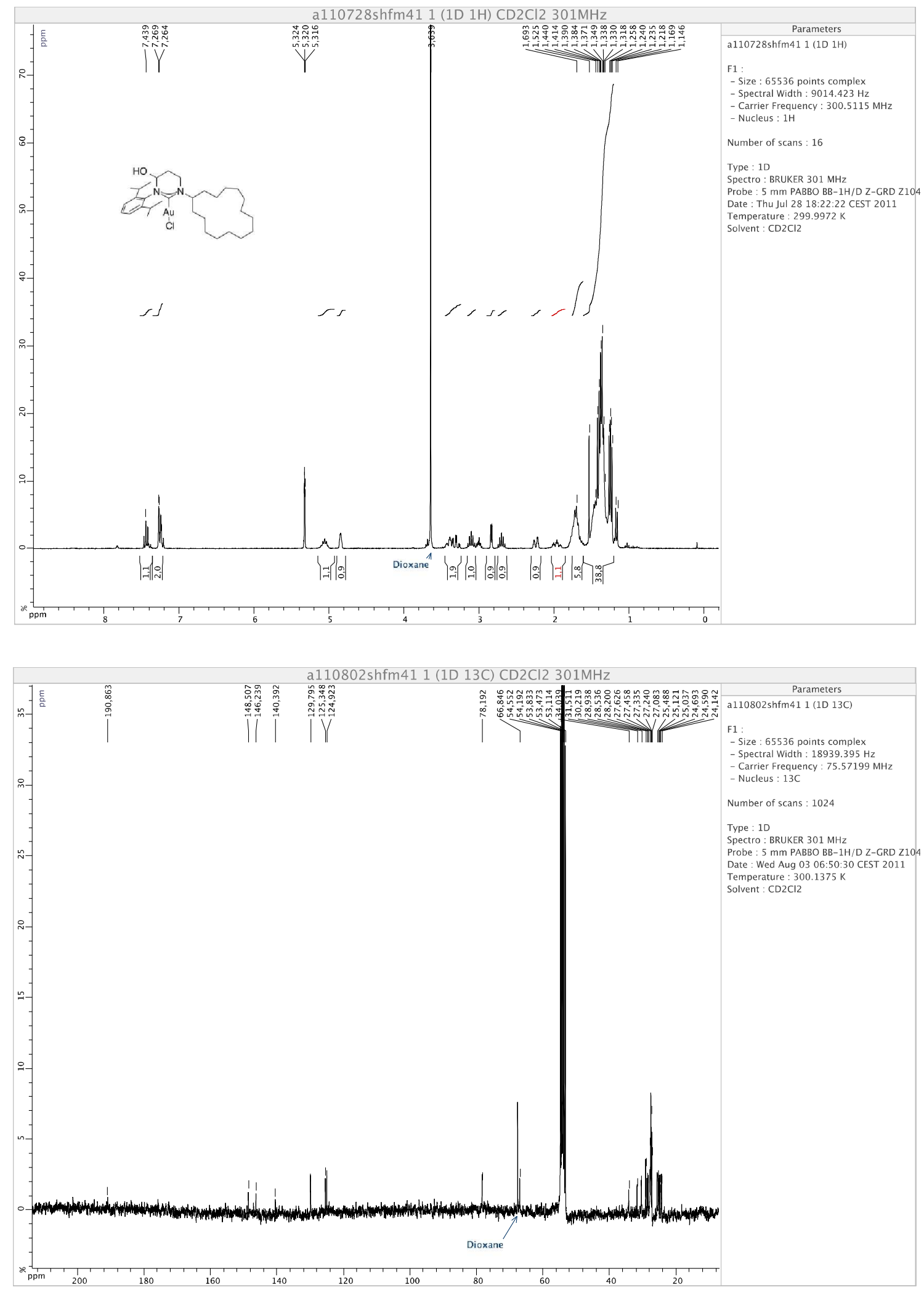

Figure S23: ${ }^{1} \mathrm{H}$ NMR spectrum (top) and ${ }^{13} \mathrm{C}$ NMR spectrum (bottom) of compound 16c 

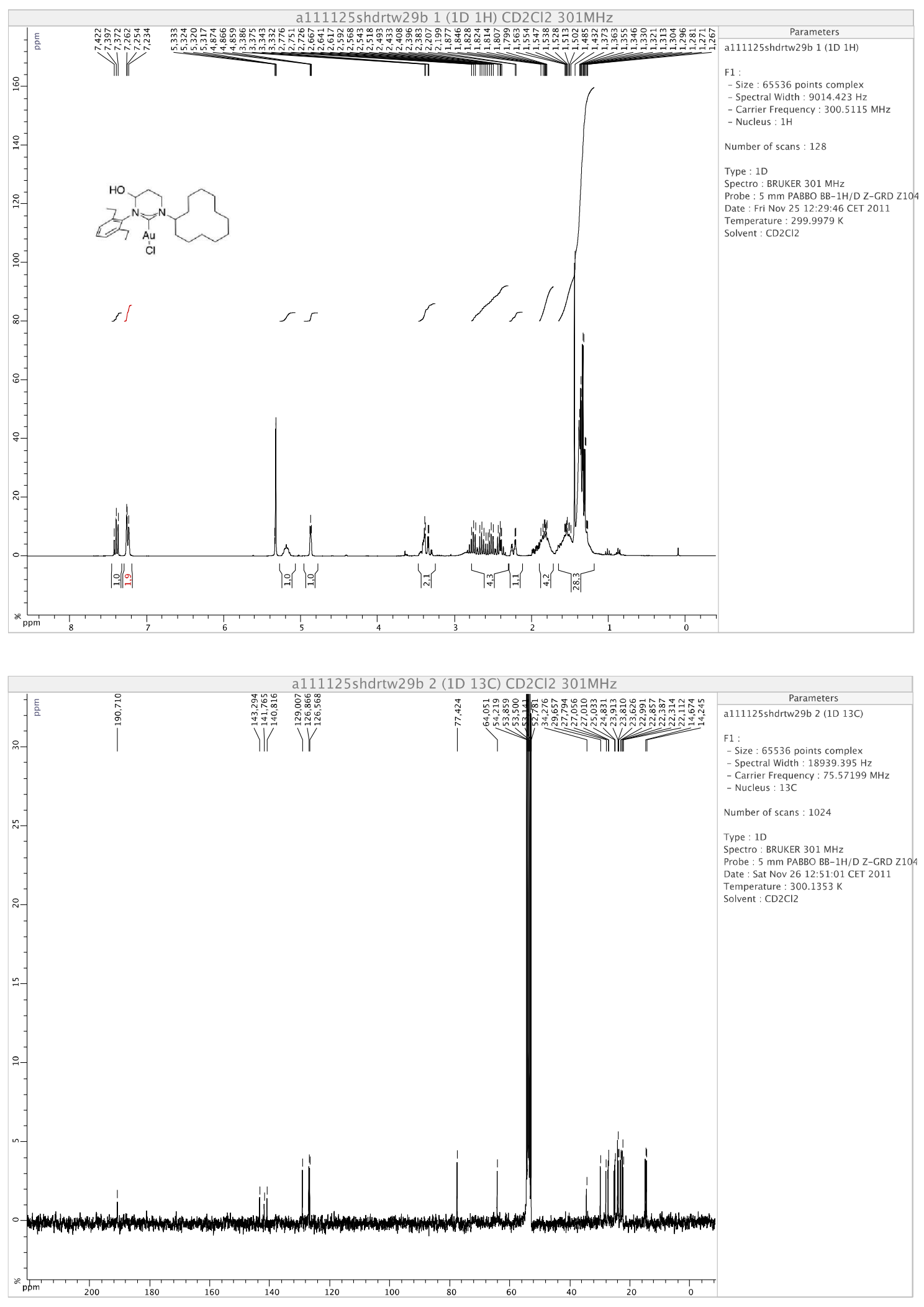

Figure S24: ${ }^{1} \mathrm{H}$ NMR spectrum (top) and ${ }^{13} \mathrm{C}$ NMR spectrum (bottom) of compound 16d 

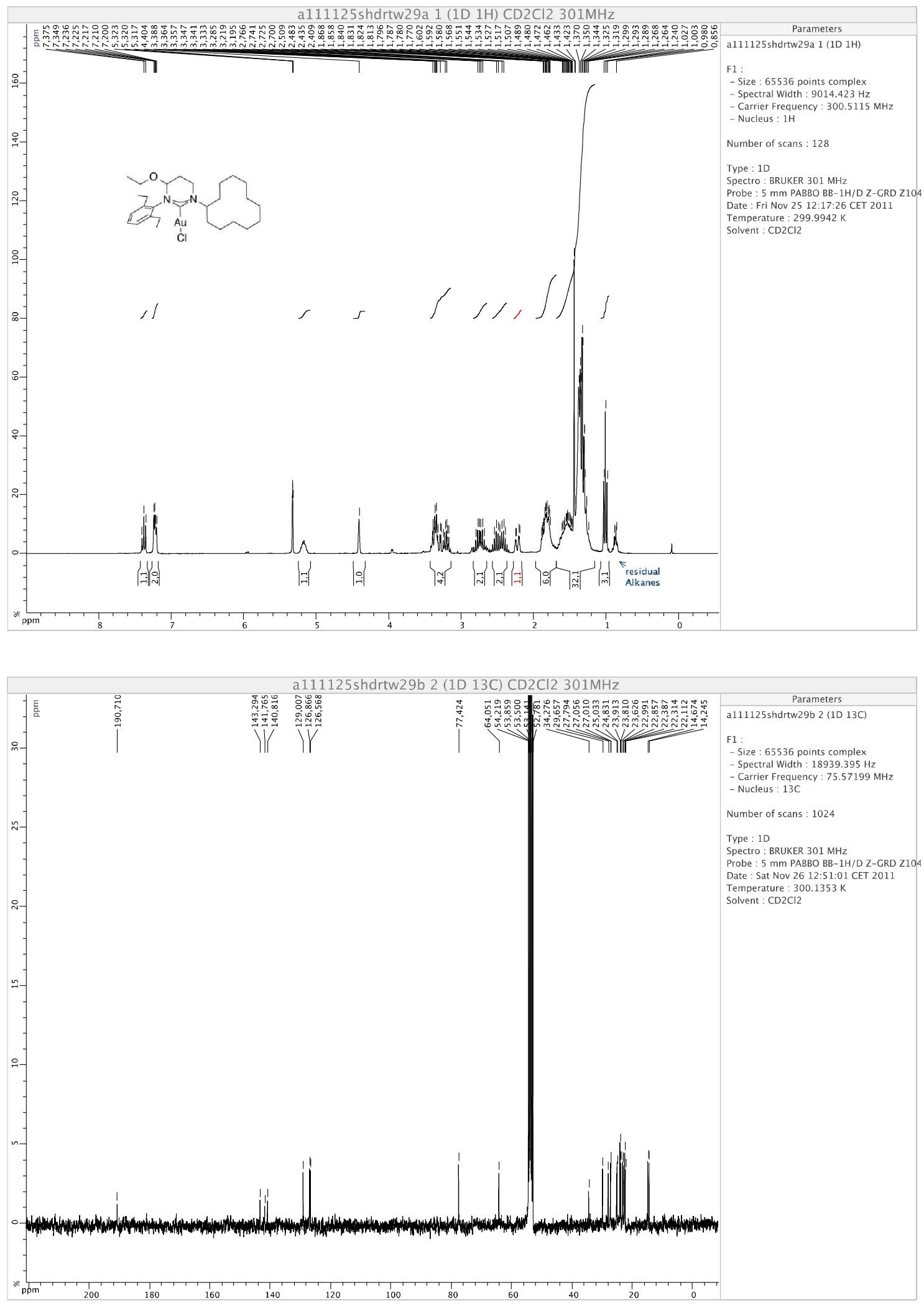

Figure S25: ${ }^{1} \mathrm{H}$ NMR spectrum (top) and ${ }^{13} \mathrm{C}$ NMR spectrum (bottom) of compound 19a 

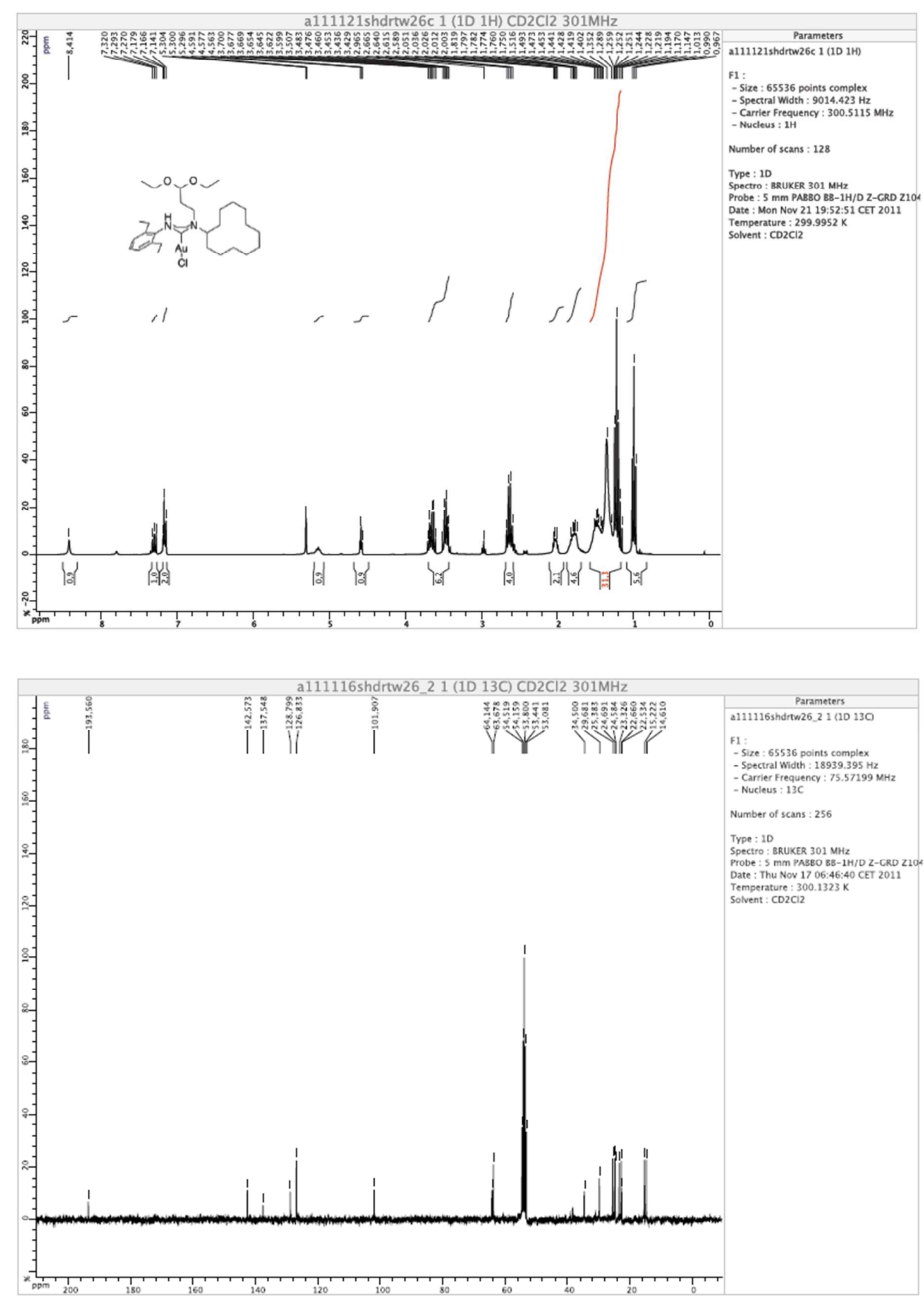

Figure S26: ${ }^{1} \mathrm{H}$ NMR spectrum (top) and ${ }^{13} \mathrm{C}$ NMR spectrum (bottom) of compound 15b 

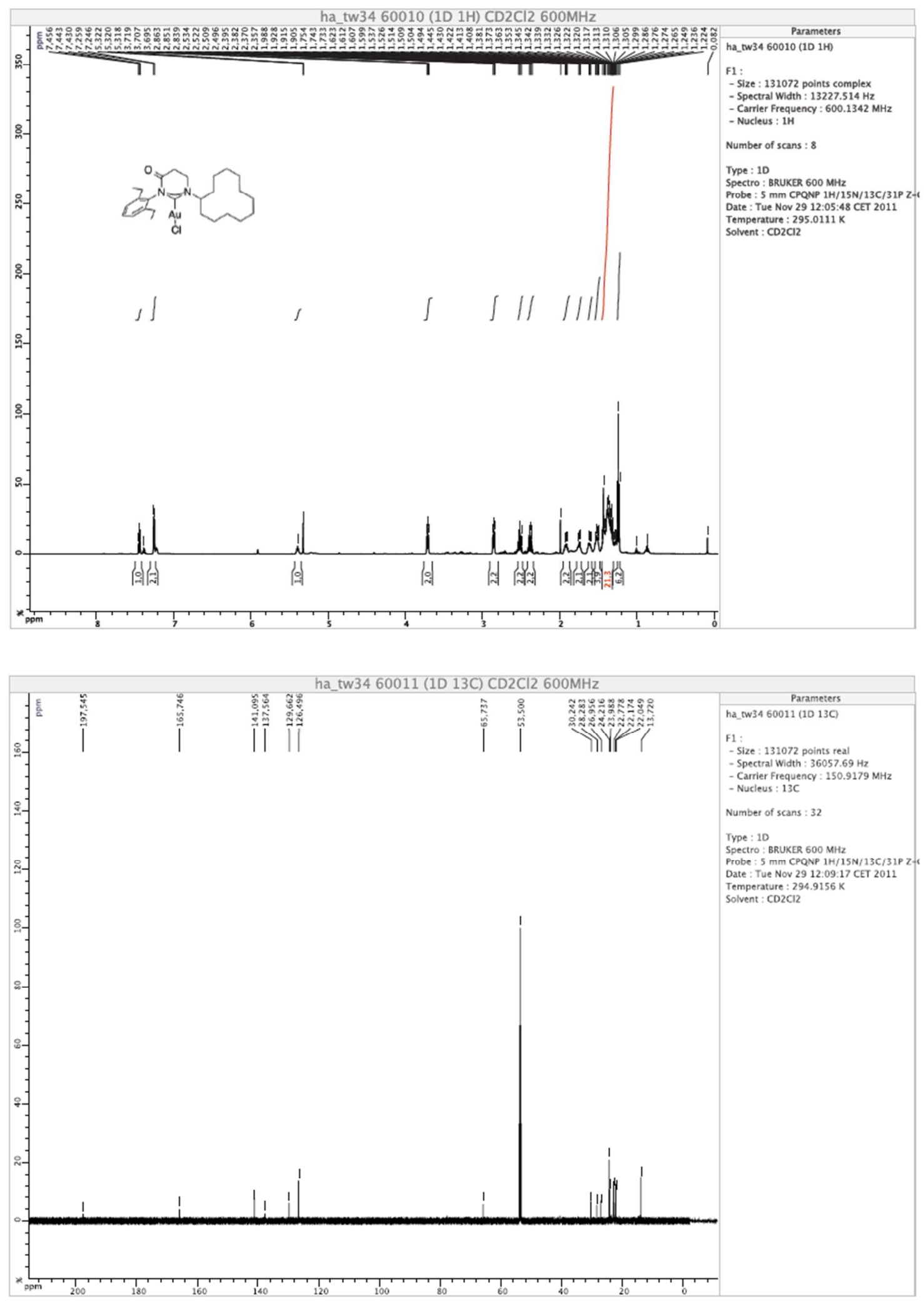

Figure S27: ${ }^{1} \mathrm{H}$ NMR spectrum (top) and ${ }^{13} \mathrm{C}$ NMR spectrum (bottom) of compound $\mathbf{1 8}$ 

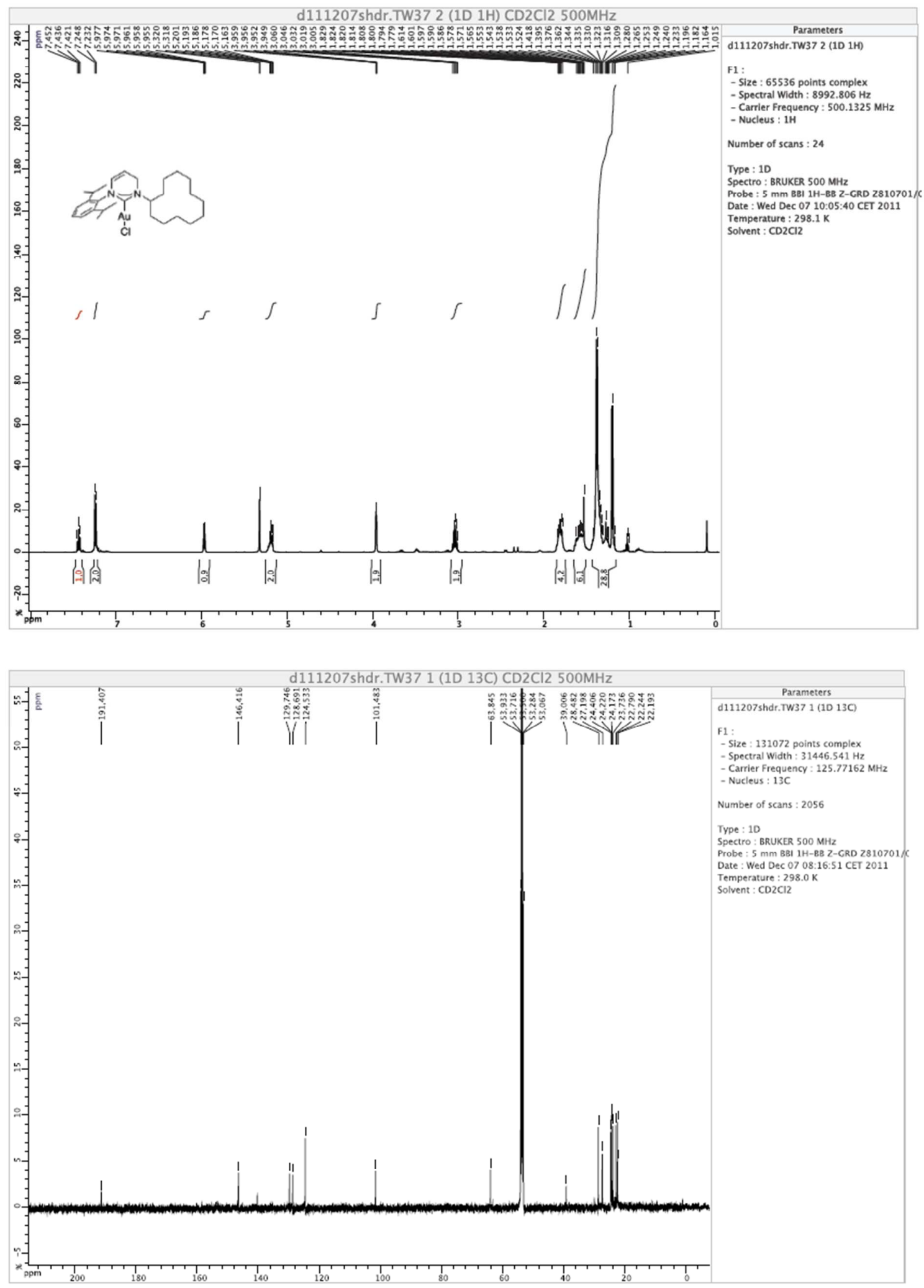

Figure S28: ${ }^{1} \mathrm{H}$ NMR spectrum (top) and ${ }^{13} \mathrm{C}$ NMR spectrum (bottom) of compound 17a 

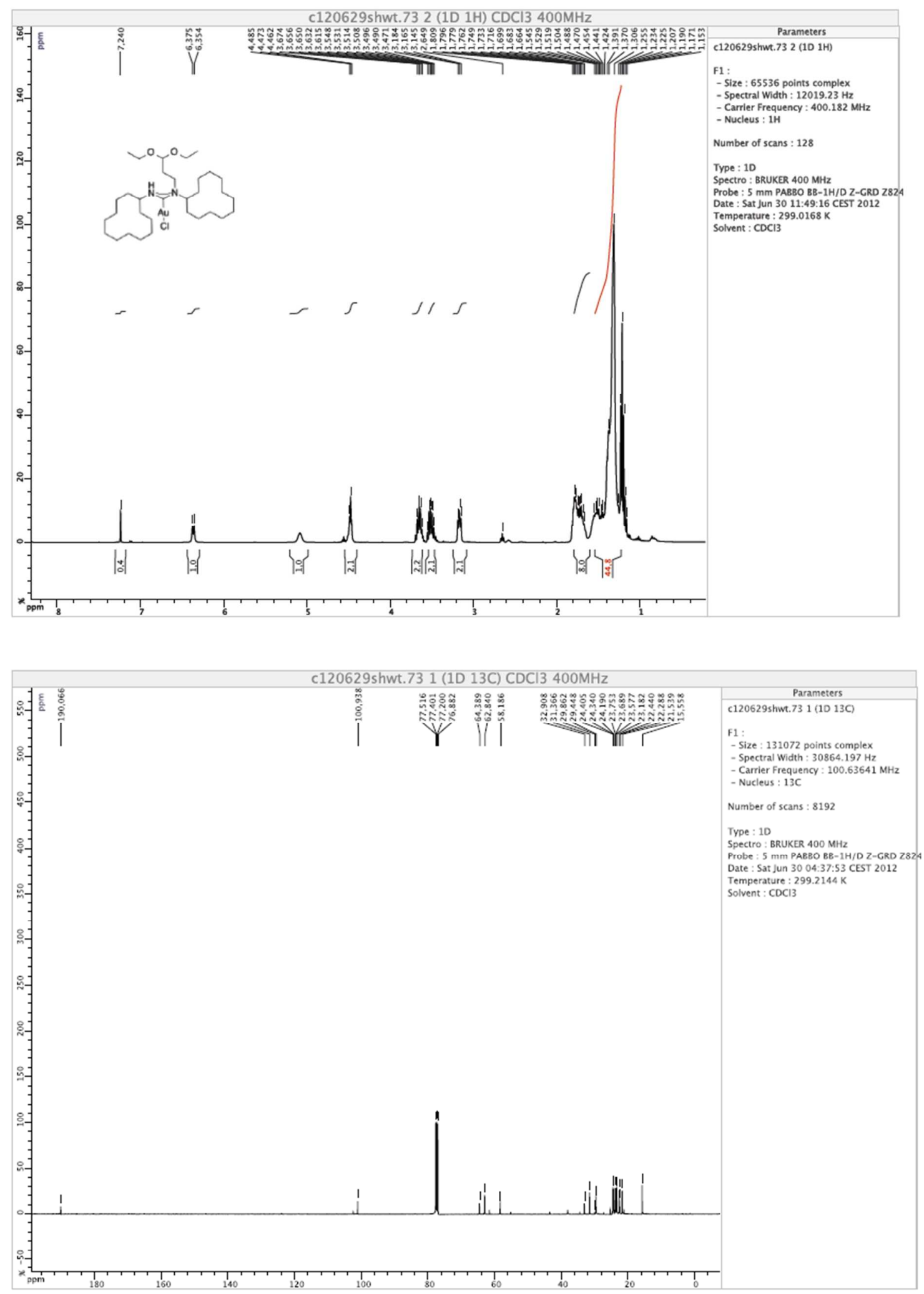

Figure S29: ${ }^{1} \mathrm{H}$ NMR spectrum (top) and ${ }^{13} \mathrm{C}$ NMR spectrum (bottom) of compound 15e 

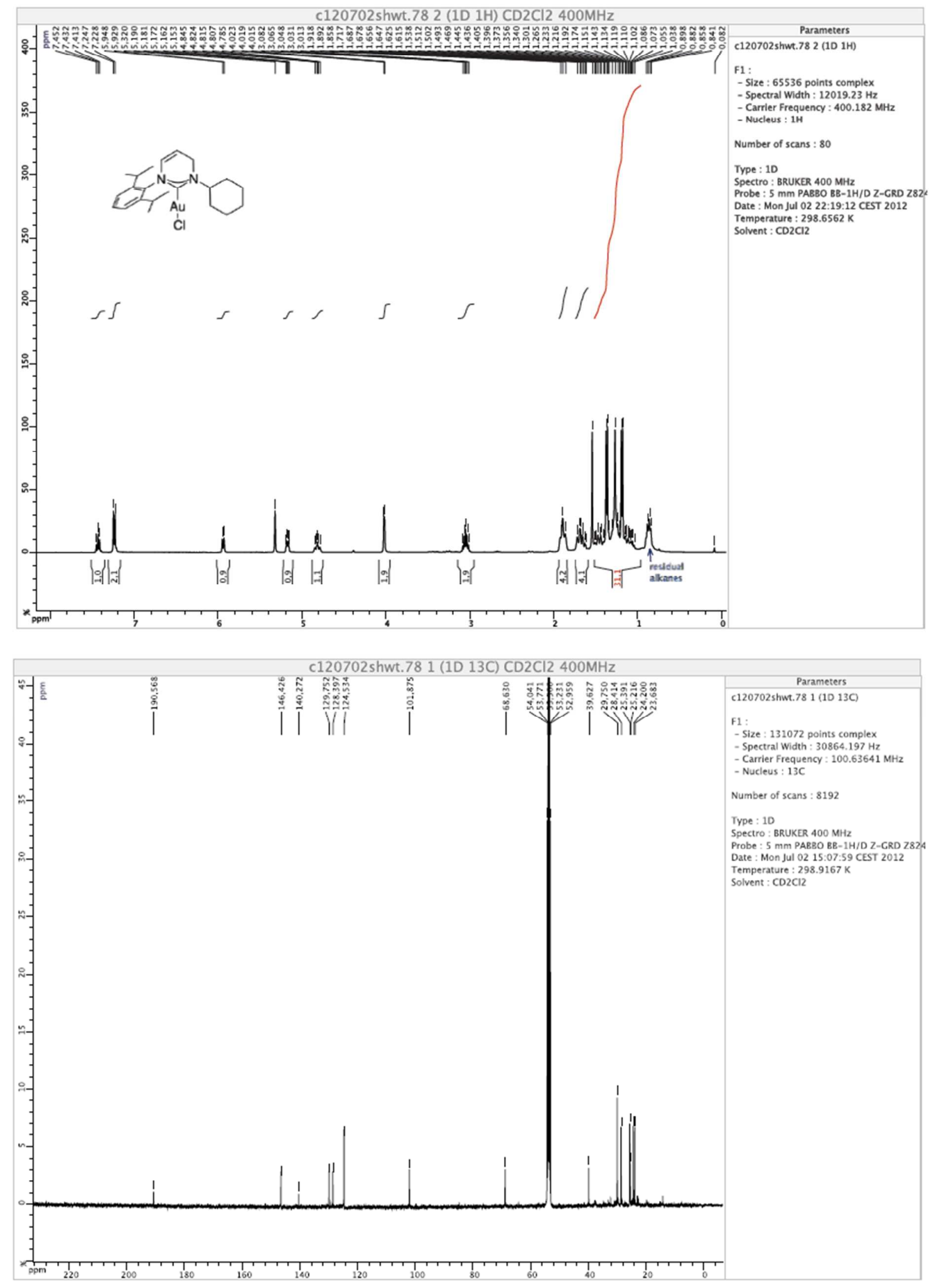

Figure S30: ${ }^{1} \mathrm{H}$ NMR spectrum (top) and ${ }^{13} \mathrm{C}$ NMR spectrum (bottom) of compound $\mathbf{1 7 b}$ 

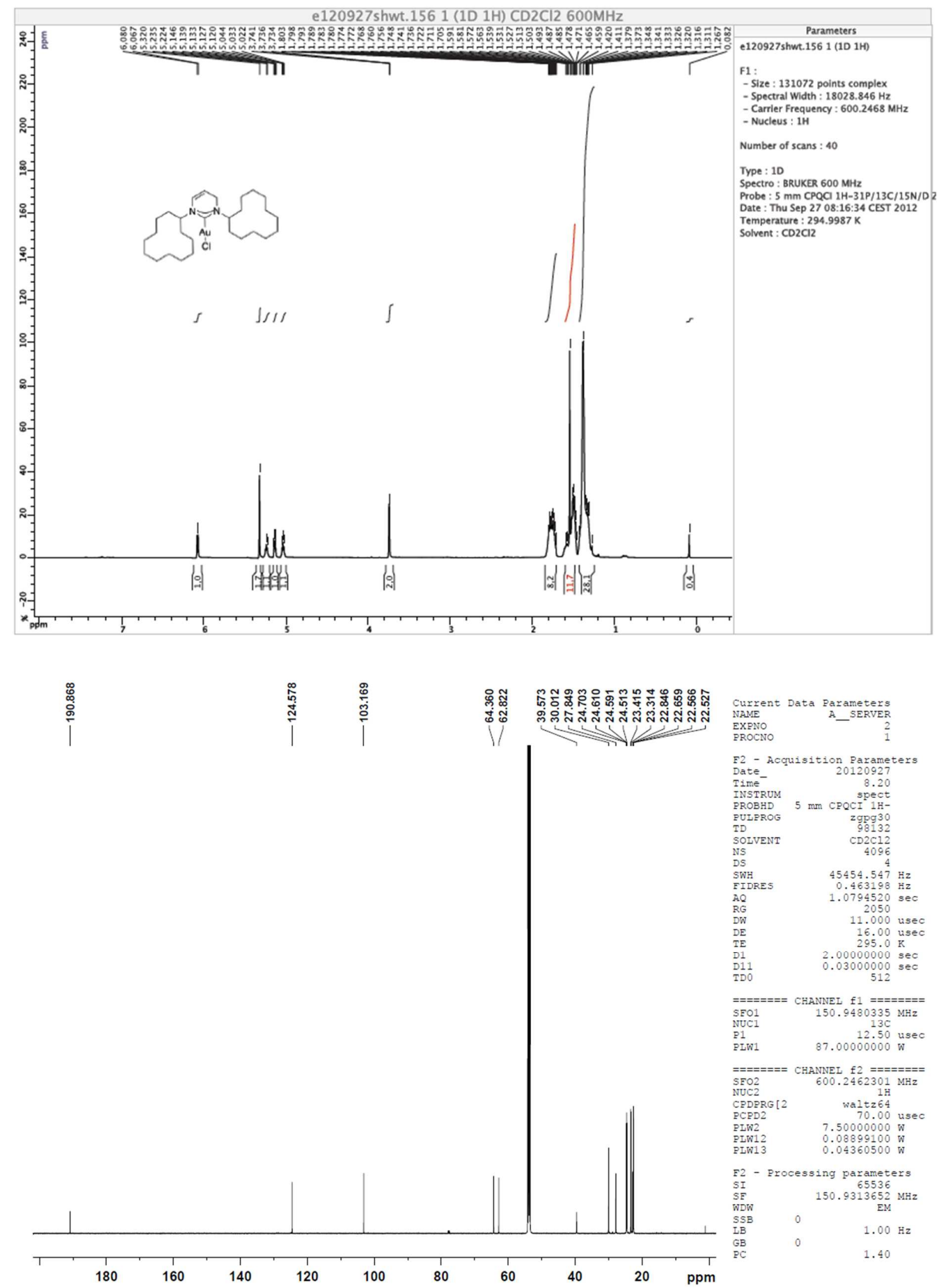

Figure S31: ${ }^{1} \mathrm{H}$ NMR spectrum (top) and ${ }^{13} \mathrm{C}$ NMR spectrum (bottom) of compound 17c 


\section{Computational Details}

\subsection{Computational Methods}

All quantumchemical calculations were performed by employing the Gaussian 09 program package. ${ }^{[5]}$ The theoretical approach is based on density functional methodologies ${ }^{[6]}$ using the B3LYP ${ }^{[7]}$ and M06 ${ }^{[8]}$ functional. Dispersion effects were treated according to Grimme. ${ }^{[9]}$ As Basis sets the correlation-consistent triple- $\zeta$-basis (cc-pVTZ) was used. ${ }^{[10]}$ For gold, additional relativistic effective core potentials from the Stuttgart group were employed. ${ }^{[11]}$ The geometries of the regarded species were, eighter in the gas phase or in solution, fully optimized using the representative computational method and checked by frequency analysis to not exhibt any imaginary frequency. Solvent effects were either described by the usage of $\mathrm{IEFPCM}^{[12]}$ or $\mathrm{SMD}^{[13]}$ calculations. For the calculations the parametrisation for dichloromethane implemented in gaussian was used. The thermochemistry of the reactions was, as already mentioned in the text, calculated at $25^{\circ} \mathrm{C}(298.15 \mathrm{~K})$ and $1 \mathrm{~atm}$.

The naming of the attached XYZ-files is directed by the geometry numbering in the following tables. 


\subsection{Computational Results}

Table S1: Overview of the obtained thermochemical data given in Ha for the geometries 1, 2, 3, 4 (B3LYP-d3 calculations)
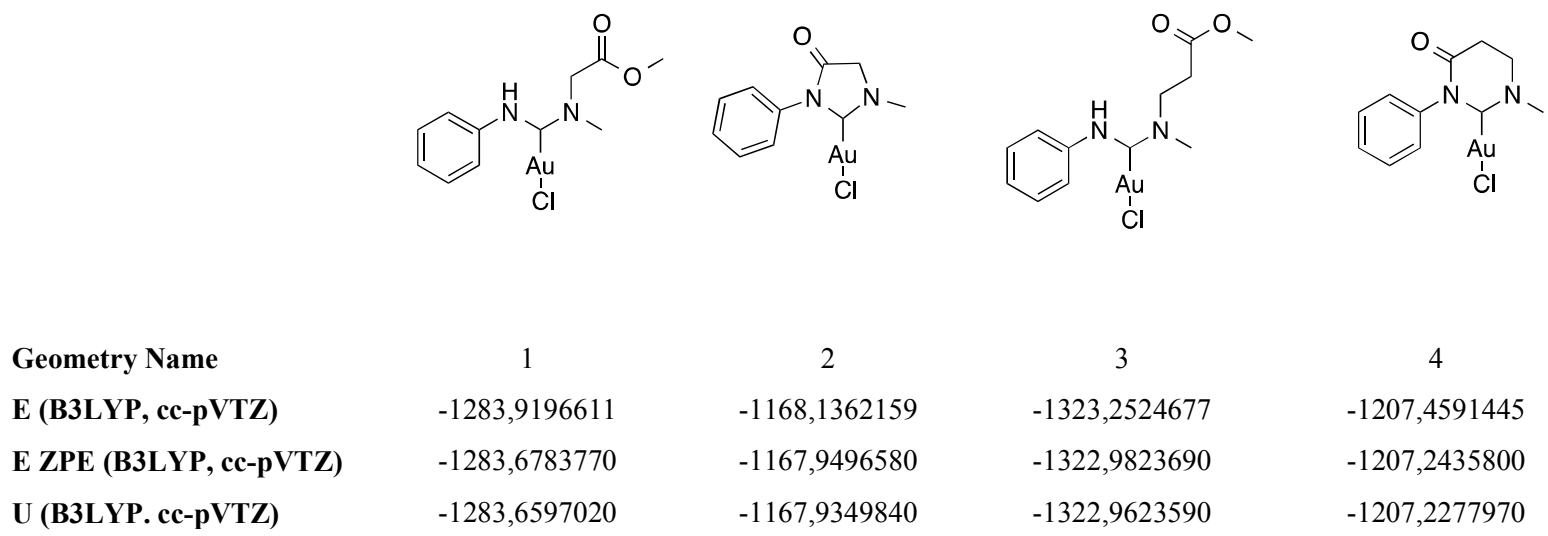
Table S2: Overview of the obtained thermochemical data given in Ha for the geometries 5, 6, 7, 8 (B3LYP-d3 calculations)
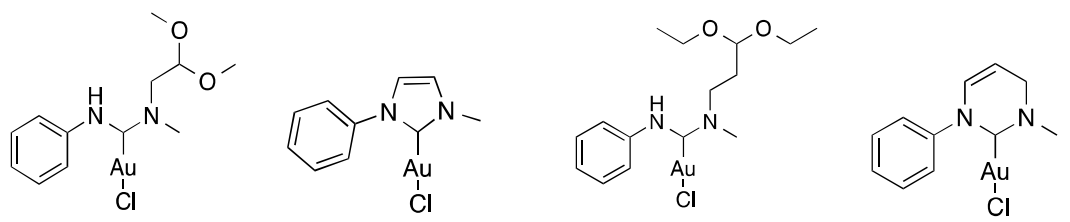

Geometry Name

E (B3LYP, cc-pVTZ)

E ZPE (B3LYP, cc-pVTZ)

U (B3LYP. cc-pVTZ)

H (B3LYP, cc-pVTZ)

G (B3LYP, cc-pVTZ)

E (B3LYP, cc-pVTZ,

PCM)
E ZPE (B3LYP, cc-pVTZ,

PCM)

U (B3LYP. cc-pVTZ,

PCM)

H (B3LYP, cc-pVTZ,

PCM)

G (B3LYP, cc-pVTZ,

PCM)

E (B3LYP, cc-pVTZ, SMD)

E ZPE (B3LYP, cc-pVTZ,

SMD)

U (B3LYP. cc-pVTZ,

SMD)

H (B3LYP, cc-pVTZ,

SMD)

G (B3LYP, cc-pVTZ,

SMD)
5

$-1324,4277613$

$-1324,1355170$

$-1324,1148550$

$-1324,1139110$

$-1324,1895980$

$-1324,4493482$

$-1324,1571380$

$-1324,1364050$

$-1324,1354610$

$-1324,2110710$

$-1324,4672195$

$-1324,1745000$

$-1324,1541190$

$-1324,1531750$

$-1324,2269810$
$-1092,8717723$

$-1092,6891770$

$-1092,6755180$

$-1092,6745740$

$-1092,7341880$

$-1092,8913234$

$-1092,7087070$

$-1092,6959050$

$-1092,6949610$

$-1092,7513050$

$-1092,9079874$

$-1092,7250910$

$-1092,7116220$

$-1092,7106780$

$-1092,7683950$
7

$-1442,4287131$

$-1442,0513950$

$-1442,0267210$

$-1442,0257770$

$-1442,1121850$

$-1442,4522750$

$-1442,0747050$

$-1442,0501110$

$-1442,0491660$

$-1442,1346840$

$-1442,4744541$

$-1442,0974640$

$-1442,0728270$

$-1442,0718830$

$-1442,1575390$
$-1132,1758261$

$-1131,9653520$

$-1131,9503160$

$-1131,9493720$

$-1132,0121660$

$-1132,1948458$

$-1131,9842090$

$-1131,9691790$

$-1131,9682350$

$-1132,0304280$

$-1132,2121959$

$-1132,0015470$

$-1131,9866340$

$-1131,9856890$

$-1132,0468900$ 
Table S3: Overview of the obtained thermochemical data given in Ha for the geometries 9, 10, 11, 12 (B3LYP-d3 calculations)

Geometry Name
E (B3LYP, cc-pVTZ)
E ZPE (B3LYP, cc-pVTZ)
U (B3LYP. cc-pVTZ)
H (B3LYP, cc-pVTZ)
G (B3LYP, cc-pVTZ)
E (B3LYP, cc-pVTZ,
PCM)
E ZPE (B3LYP, cc-pVTZ,
PCM)
U (B3LYP. cc-pVTZ,
PCM)
H (B3LYP, cc-pVTZ,
PCM)
G (B3LYP, cc-pVTZ,
PCM)
E (B3LYP, cc-pVTZ, SMD)
E ZPE (B3LYP, cc-pVTZ,
SMD)
U (B3LYP. cc-pVTZ,
SMD)
H (B3LYP, cc-pVTZ,
SMD)
G (B3LYP, cc-pVTZ,
SMD)<smiles>CN1CC(O)N(c2ccccc2)C1[Al]Cl</smiles>

9<smiles>CN1CCC(O)N(c2ccccc2)C1[Al]Cl</smiles>

10

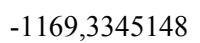

$-1169,1243750$

$-1169,1090880$

$-1169,1081440$

$-1169,1699760$

$-1169,3559121$

$-1.169,145819$

$-1169,130441$

$-1169,129496$

$-1169,191636$

$-1169,3728629$

$-1169,1630450$

$-1169,1477190$

$-1169,1467750$

$-1169,2083840$

$-1208,6637195$

$-1208,4242780$

$-1208,4080140$

$-1208,4070690$

$-1208,4713730$

$-1208,6852115$

$-1208,4459690$

$-1208,4295610$

$-1208,4286170$

$-1208,4932370$

$-1208,7027369$

$-1208,4638010$

$-1208,4474040$

$-1208,4464600$

$-1208,5107750$<smiles>COC1CN(C)C([Al]Cl)N1c1ccccc1</smiles>

11<smiles>CCOC1CCN(C)C([Al]Cl)N1c1ccccc1</smiles>

12

$-1287,3140471$

$-1287,0185370$

$-1286,9994250$

$-1286,9984800$

$-1287,0700500$

$-1.287,3349365$

$-1287,0395140$

$-1287,0203250$

$-1287,0193810$

$-1287,0913460$

$-1287,3534814$

$-1287,0582330$

$-1287,0391370$

$-1287,0381930$

$-1287,1093600$ 
Table S4: Overview of the obtained thermochemical data given in Ha for the geometries 13, 14, 15 (B3LYP-d3 calculations)

$\mathrm{MeOH} \quad \mathrm{EtOH} \quad \mathrm{H} 2 \mathrm{O}$

$\begin{array}{lccc}\text { Geometry Name } & 13 & 14 & 15 \\ \text { E (B3LYP, cc-pVTZ) } & -115,7733618 & -155,1091825 & -76,4598478 \\ \text { E ZPE (B3LYP, cc-pVTZ) } & -115,7223370 & -155,0295110 & -76,4385590 \\ \text { U (B3LYP. cc-pVTZ) } & -115,7189990 & -155,0252050 & -76,4357240 \\ \text { H (B3LYP, cc-pVTZ) } & -115,7180550 & -155,0242600 & -76,4347800 \\ \text { G (B3LYP, cc-pVTZ) } & -115,7450970 & -155,0549140 & -76,4562010 \\ & & & \\ \text { E (B3LYP, cc-pVTZ, PCM) } & -115,7773732 & -155,1130167 & -76,4654373 \\ \text { E ZPE (B3LYP, cc-pVTZ, PCM) } & -115,7264050 & -155,0334990 & -76,4442290 \\ \text { U (B3LYP. cc-pVTZ, PCM) } & -115,7230610 & -155,0291680 & -76,4413940 \\ \text { H (B3LYP, cc-pVTZ, PCM) } & -115,7221170 & -155,0282240 & -76,4404490 \\ \text { G (B3LYP, cc-pVTZ, PCM) } & -115,7491730 & -155,0589310 & -76,4618750 \\ & & & \\ \text { E (B3LYP, cc-pVTZ, SMD) } & -115,7781635 & -155,1157237 & -76,4666140 \\ \text { E ZPE (B3LYP, cc-pVTZ, SMD) } & -115,7272500 & -155,0364300 & -76,4455990 \\ \text { U (B3LYP. cc-pVTZ, SMD) } & -115,7239260 & -155,0321040 & -76,4427630 \\ \text { H (B3LYP, cc-pVTZ, SMD) } & -115,7229820 & -155,0311600 & -76,4418190 \\ \text { G (B3LYP, cc-pVTZ, SMD) } & -115,7499980 & -155,0618500 & -76,4632500\end{array}$


Table S5: Overview of the obtained thermochemical data given in Ha for the geometries 1, 2, 3, 4 (M06 calculations)

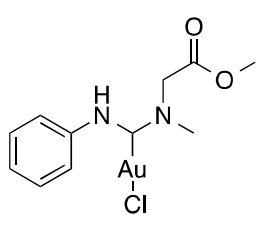

Geometry Name

E (M06, cc-pVTZ)

E ZPE (M06, cc-pVTZ)

U (M06. cc-pVTZ)

H (M06, cc-pVTZ)

G (M06, cc-pVTZ)

E (M06, cc-pVTZ, PCM)

E ZPE (M06, cc-pVTZ,

PCM)

U (M06. cc-pVTZ, PCM)

H (M06, cc-pVTZ, PCM)

G (M06, cc-pVTZ, PCM)

E (M06, cc-pVTZ, SMD)

E ZPE (M06, cc-pVTZ,

SMD)

U (M06. cc-pVTZ, SMD)

H (M06, cc-pVTZ, SMD)

G (M06, cc-pVTZ, SMD)<smiles>CN1CC(=O)N(c2ccccc2)C1[Al]Cl</smiles>

2

$-1283,3437351$

$-1283,1029670$

$-1283,0843990$

$-1283,0834550$

$-1283,1548890$

$-1.283,367934$

$-1.283,127429$

$-1.283,108791$

$-1.283,107847$

$-1.283,179138$

$-1283,3858863$

$-1283,1449930$

$-1283,1265820$

$-1283,1256370$

$-1283,1955100$<smiles>COC(=O)CCN(C)C(Nc1ccccc1)[Al]Cl</smiles>

3

$-1322,6402736$

$-1322,3712330$

$-1322,3521640$

$-1322,3512200$

$-1322,4231570$

$-1322,6650827$

$-1322,3954300$

$-1322,3756890$

$-1322,3747450$

$-1322,4482600$

$-1322,6839764$

$-1322,4138180$

$-1322,3942850$

$-1322,3933400$

$-1322,4659640$

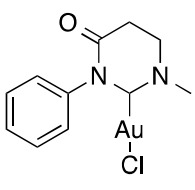

4

$-1206,9250358$

$-1206,7103880$

$-1206,6945170$

$-1206,6935730$

$-1206,7570240$

$-1206,9489157$

$-1206,7342250$

$-1206,7183270$

$-1206,7173830$

$-1206,7808810$

$-1206,9668268$

$-1206,7519900$

$-1206,7362430$

$-1206,7352990$

$-1206,7978910$ 
Table S6: Overview of the obtained thermochemical data given in Ha for the geometries 5, 6, 7, 8 (M06 calculations)<smiles>COC(CN(C)C(NCl)Nc1ccccc1)OC</smiles>

5

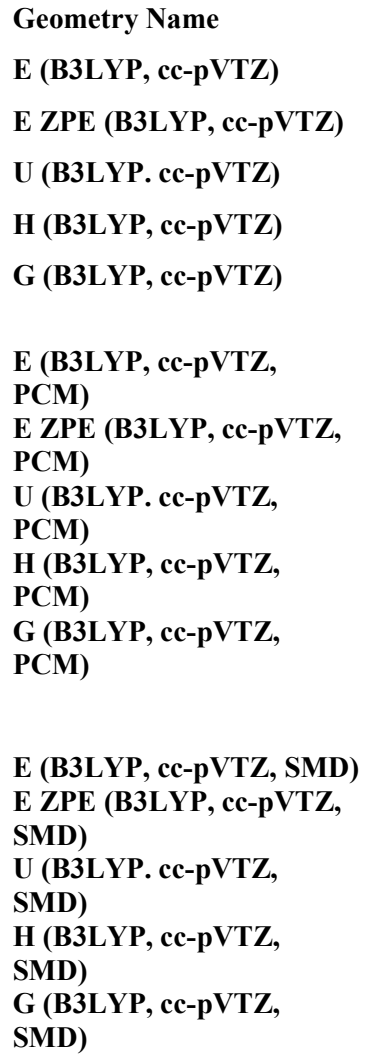<smiles>CN1C=CN(c2ccccc2)C1[Al]Cl</smiles><smiles>CCOC(CCN(C)C(Nc1ccccc1)[Al]Cl)OCC</smiles><smiles>CN1CC=CN(c2ccccc2)C1[Al]Cl</smiles>

6

$$
-1092,4041415
$$$$
-1092,2227200
$$$$
-1092,2089530
$$$$
-1092,2080090
$$$$
-1092,2673380
$$

7

$-1441,6999122$

$-1441,3236720$

$-1441,2993090$

$-1441,2983650$

$-1441,3824720$

$-1441,7228615$

$-1441,3468250$

$-1441,3224100$

$-1441,3214660$

$-1441,4057830$

$-1441,7449742$

$-1441,3687680$

$-1441,3445620$

$-1441,3436180$

$-1441,4270260$
$-1131,6736436$

$-1131,4638330$

$-1131,4489140$

$-1131,4479700$

$-1131,5094440$

$-1131,6927088$

$-1131,4827860$

$-1131,4678860$

$-1131,4669420$

$-1131,5280650$

$-1131,7097401$

$-1131,4999290$

$-1131,4850270$

$-1131,4840830$

$-1131,5450040$ 
Table S7: Overview of the obtained thermochemical data given in Ha for the geometries 9, 10, 11, 12 (M06 calculations)

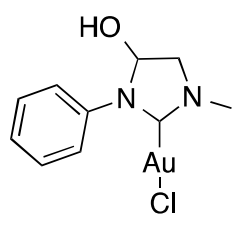

Geometry Name
E (B3LYP, cc-pVTZ)

E ZPE (B3LYP, cc-pVTZ)

U (B3LYP. cc-pVTZ)

H (B3LYP, cc-pVTZ)

G (B3LYP, cc-pVTZ)

E (B3LYP, cc-pVTZ,

PCM)

PCM)

U (B3LYP. cc-pVTZ,

PCM)

H (B3LYP, cc-pVTZ,

PCM)

G (B3LYP, cc-pVTZ,

PCM)

E (B3LYP, cc-pVTZ, SMD)

E ZPE (B3LYP, cc-pVTZ,

SMD)

U (B3LYP. cc-pVTZ,

SMD)

H (B3LYP, cc-pVTZ,

SMD)

G (B3LYP, cc-pVTZ,

SMD)

$-1168,6414400$
9

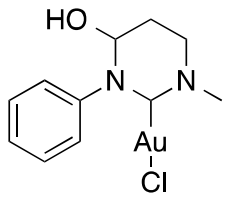

10

$-1168,8291478$

$-1168,6198690$

$-1168,6045250$

$-1168,6035800$

$-1168,6654610$

$-1168,8504999$

$-1168,6259340$

$-1168,6249900$

$-1168,6873150$

$-1208,1240060$

$-1207,8852490$

$-1207,8690410$

$-1207,8680970$

$-1207,9318540$

$-1208,1455372$

$-1207,9070300$

$-1207,8907010$

$-1207,8897570$

$-1207,9538230$

$-1208,1628078$

$-1207,9247190$

$-1207,9083310$

$-1207,9073860$

$-1207,9718430$<smiles>COC1CN(C)C([Al]Cl)N1c1ccccc1</smiles>

11

$-1208,1075345$

$-1207,8705550$

$-1207,8538260$

$-1207,8528810$

$-1207,9177850$

$-1208,1284048$

$-1207,8914060$

$-1207,8746330$

$-1207,8736890$

$-1207,9386170$

$-1208,1447818$

$-1207,9081810$

$-1207,8913130$

$-1207,8903690$

$-1207,9558000$<smiles>CCOC1CCN(C)C([Al]Cl)N1c1ccccc1</smiles>

12

$-1286,7017530$

$-1286,4074950$

$-1286,3884230$

$-1286,3874790$

$-1286,4587330$

$-1286,7227191$

$-1286,4283560$

$-1286,4093190$

$-1286,4083750$

$-1286,4792250$

$-1286,7409762$

$-1286,4468710$

$-1286,4278580$

$-1286,4269140$

$-1286,4976070$ 
Table S8: Overview of the obtained thermochemical data given in Ha for the geometries 13, 14, 15, 16 (M06 calculations)

$\mathrm{MeOH} \quad \mathrm{EtOH} \quad \mathrm{H} 2 \mathrm{O}$

\begin{tabular}{lccc} 
Geometry Name & 13 & 14 & 15 \\
E (B3LYP, cc-pVTZ) & $-115,6935341$ & $-154,9924582$ & $-76,4175126$ \\
E ZPE (B3LYP, cc-pVTZ) & $-115,6423530$ & $-154,9130490$ & $-76,3958670$ \\
U (B3LYP. cc-pVTZ) & $-115,6390210$ & $-154,9086930$ & $-76,3930320$ \\
H (B3LYP, cc-pVTZ) & $-115,6380770$ & $-154,9077490$ & $-76,3920870$ \\
G (B3LYP, cc-pVTZ) & $-115,6650880$ & $-154,9384850$ & $-76,4134980$ \\
& & & \\
E (B3LYP, cc-pVTZ, PCM) & $-115,6974565$ & $-154,9962673$ & $-76,4230839$ \\
E ZPE (B3LYP, cc-pVTZ, & $-115,6463480$ & $-154,9170190$ & $-76,4015500$ \\
PCM) & $-115,6430090$ & $-154,9126400$ & $-76,3987140$ \\
U (B3LYP. cc-pVTZ, PCM) & $-115,6420650$ & $-154,9116960$ & $-76,3977700$ \\
H (B3LYP, cc-pVTZ, PCM) & $-115,6690920$ & $-154,9424840$ & $-76,4191860$ \\
G (B3LYP, cc-pVTZ, PCM) & & & \\
& & $-154,9989158$ & $-76,4242402$ \\
E (B3LYP, cc-pVTZ, SMD) & $-115,6981999$ & $-154,9199680$ & $-76,4029160$ \\
E ZPE (B3LYP, cc-pVTZ, & $-115,6472400$ & $-154,9155940$ & $-76,4000810$ \\
SMD) & $-115,6439200$ & $-154,9146490$ & $-76,3991360$ \\
U (B3LYP. cc-pVTZ, SMD) & $-115,6429750$ & $-154,9454180$ & $-76,4205590$ \\
H (B3LYP, cc-pVTZ, SMD) & $-115,6699610$ & & \\
G (B3LYP, cc-pVTZ, SMD) & & & \\
& & & \\
(BMD & & & \\
(B) & & & \\
\hline
\end{tabular}


Table S9: Overview of the obtained thermochemical data given in $\mathrm{kcal} / \mathrm{mol}$ for the NHOC ring closure reactions

\begin{tabular}{|c|c|c|c|c|}
\hline ring closure NHOC & 5 ring & 6 ring & 5 ring $P C M$ & 6 ring $P C M$ \\
\hline E (B3LYP, cc-pVTZ) & 6,3222918000 & 12,5157977999 & 5,2345138890 & 10,7149641390 \\
\hline E ZPE (B3LYP, cc-pVTZ) & 4,0015140000 & 10,3154040000 & 2,9149230000 & 8,4544679999 \\
\hline U (B3LYP. cc-pVTZ) & 3,5858130000 & 9,7580010001 & 2,5036110000 & 7,9277880001 \\
\hline H (B3LYP, cc-pVTZ) & 4,1777009999 & 10,3505160000 & 3,0961260000 & 8,5196760000 \\
\hline G (B3LYP, cc-pVTZ) & $-5,8311000000$ & 0,8953560000 & $-7,1666100000$ & $-1,0571219999$ \\
\hline E (M06, cc-pVTZ) & 7,9625238000 & 13,6082199000 & 6,9598304160 & 11,7314822460 \\
\hline E ZPE (M06, cc-pVTZ) & 5,5370370001 & 11,5944840001 & 4,8147329999 & 9,3153390001 \\
\hline U (M06. cc-pVTZ) & 5,1934410001 & 11,6785020000 & 4,4134530000 & 8,9993310000 \\
\hline H (M06, cc-pVTZ) & 5,7853289999 & 12,2703900000 & 5,0053410000 & 9,5912190001 \\
\hline G (M06, cc-pVTZ) & $-4,1952570000$ & 0,6552150000 & $-5,0805810001$ & $-1,0740510001$ \\
\hline$\underline{\text { ring closure NHOC }}$ & 5 ring SMD & 6 ring SMD & & \\
\hline E (B3LYP, cc-pVTZ) & 5,1602106269 & 10,7490378270 & & \\
\hline E ZPE (B3LYP, cc-pVTZ) & 2,5556520000 & 8,2450500000 & & \\
\hline U (B3LYP. cc-pVTZ) & 2,2396439999 & 7,8042689999 & & \\
\hline H (B3LYP, cc-pVTZ) & 2,8315320000 & 8,3955300000 & & \\
\hline G (B3LYP, cc-pVTZ) & $-8,0487990000$ & $-1,1850300000$ & & \\
\hline E (M06, cc-pVTZ) & 6,9404912280 & 11,8814706780 & & \\
\hline E ZPE (M06, cc-pVTZ) & 4,3701900001 & 9,1466760000 & & \\
\hline U (M06. cc-pVTZ) & 4,0767540001 & 8,8544940000 & & \\
\hline H (M06, cc-pVTZ) & 4,6686420000 & 9,4463820001 & & \\
\hline G (M06, cc-pVTZ) & $-6,0486690001$ & $-1,1837760000$ & & \\
\hline
\end{tabular}


Table S10: Overview of the thermochemical data given in $\mathrm{kcal} / \mathrm{mol}$ for the ring closure reactions to the unsaturated NHC gold chlorides.

\begin{tabular}{|c|c|c|c|c|}
\hline ring closure unsaturated & 5 ring & 6 ring & 5 ring $\mathrm{PCM}$ & 6 ring $\mathrm{PCM}$ \\
\hline E (B3LYP, cc-pVTZ) & 5,809405800002 & 21,645294000095 & 2,055553038039 & 19,685251871908 \\
\hline E ZPE (B3LYP, cc-pVTZ) & 1,044581999929 & 16,942166999923 & $-2,745632999982$ & 14,733246000011 \\
\hline U (B3LYP. cc-pVTZ) & 0,839553000090 & 16,298864999979 & $-3,524994000082$ & 14,167692000013 \\
\hline H (B3LYP, cc-pVTZ) & 2,023328999953 & 17,483894999981 & $-2,341217999934$ & 15,350840999949 \\
\hline G (B3LYP, cc-pVTZ) & 21,809567999966 & $-6,150243000083$ & $24,189660000087^{-}$ & $-8,530961999988$ \\
\hline E (M06, cc-pVTZ) & 11,075014500159 & 25,927829399932 & 7,222742802018 & 23,586531143992 \\
\hline E ZPE (M06, cc-pVTZ) & 6,317652000017 & 21,155606999977 & 2,271621000039 & 18,810627000053 \\
\hline U (M06. cc-pVTZ) & 6,291945000010 & 20,696642999995 & 1,818300000114 & 18,335988000039 \\
\hline H (M06, cc-pVTZ) & 7,475721000158 & 21,880419000143 & 3,002075999976 & 19,519764000044 \\
\hline G (M06, cc-pVTZ) & $16,767234000008^{-}$ & $-2,471634000033$ & 20,275298999919 & $-4,545750000071$ \\
\hline$\underline{\text { ring closure unsaturated }}$ & 5 ring SMD & 6 ring SMD & & \\
\hline E (B3LYP, cc-pVTZ) & 1,821574193988 & 19,318313915994 & & \\
\hline E ZPE (B3LYP, cc-pVTZ) & $-3,192056999996$ & 14,456738999925 & & \\
\hline U (B3LYP. cc-pVTZ) & $-3,357585000005$ & 13,784594999911 & & \\
\hline H (B3LYP, cc-pVTZ) & $-2,173809000000$ & 14,968997999986 & & \\
\hline G (B3LYP, cc-pVTZ) & 25,964070000026 & $-8,182977000083$ & & \\
\hline E (M06, cc-pVTZ) & 6,834511925892 & 23,451344928015 & & \\
\hline E ZPE (M06, cc-pVTZ) & 2,202024000000 & 18,122180999937 & & \\
\hline U (M06. cc-pVTZ) & 2,076624000032 & 17,773569000105 & & \\
\hline H (M06, cc-pVTZ) & 3,262281000103 & 18,958599000107 & & \\
\hline G (M06, cc-pVTZ) & 20,388785999846 & $-5,526378000018$ & & \\
\hline
\end{tabular}


Table S11: Overview of the obtained thermochemical data given in $\mathrm{kcal} / \mathrm{mol}$ for the hydration reaction of the unsaturated NHC ligands

\begin{tabular}{|c|c|c|c|c|}
\hline hydration unsaturated & 5 ring & 6 ring & 5 ring $\mathrm{PCM}$ & 6 ring $\mathrm{PCM}$ \\
\hline E (B3LYP, cc-pVTZ) & $-1,814976900044$ & 17,584591200026 & 0,532072199954 & 15,630075449989 \\
\hline E ZPE (B3LYP, cc-pVTZ) & 2,107346999883 & 12,770109000049 & 4,462358999925 & 10,991937000119 \\
\hline U (B3LYP. cc-pVTZ) & 1,350558000012 & 13,777698000143 & 4,299965999978 & 11,905476000022 \\
\hline H (B3LYP, cc-pVTZ) & 0,758669999938 & 14,368959000004 & 3,708077999905 & 12,497991000023 \\
\hline \multirow[t]{2}{*}{ G (B3LYP, cc-pVTZ) } & 12,798950999976 & $-1,884762000089$ & 13,508087999968 & $-0,585617999947$ \\
\hline & & - & & \\
\hline E (M06, cc-pVTZ) & $-4,698549900105$ & 20,596824599934 & $-2,344986270143$ & 18,649776420047 \\
\hline E ZPE (M06, cc-pVTZ) & $-0,803814000109$ & 16,019222999972 & 1,393820999974 & 14,229138000001 \\
\hline U (M06. cc-pVTZ) & $-1,592579999971$ & 16,988564999948 & 1,238324999939 & 15,111327000126 \\
\hline H (M06, cc-pVTZ) & $-2,184468000045$ & 17,581080000092 & 0,646437000007 & 15,703214999915 \\
\hline G (M06, cc-pVTZ) & 9,640124999968 & $-5,587823999863$ & 10,614483000074 & $-4,120644000003$ \\
\hline hydration unsaturated & 5 ring SMD & 6 ring SMD & & \\
\hline E (B3LYP, cc-pVTZ) & 1,090039499921 & 15,002222730040 & & \\
\hline E ZPE (B3LYP, cc-pVTZ) & 4,793414999944 & $10,442685000080^{-}$ & & \\
\hline U (B3LYP. cc-pVTZ) & 4,179581999997 & 11,290388999936 & & \\
\hline H (B3LYP, cc-pVTZ) & 3,587693999923 & 11,882903999937 & & \\
\hline G (B3LYP, cc-pVTZ) & 14,584647000030 & $-0,398144999921$ & & \\
\hline E (M06, cc-pVTZ) & $-1,783545389987$ & $\begin{array}{r}- \\
18,074823690078\end{array}$ & & \\
\hline E ZPE (M06, cc-pVTZ) & 1,887270000082 & $13,71499800001 \overline{6}^{-}$ & & \\
\hline U (M06. cc-pVTZ) & 1,253373000043 & 14,560821000090 & & \\
\hline H (M06, cc-pVTZ) & 0,660230999973 & 15,152709000021 & & \\
\hline G (M06, cc-pVTZ) & 11,803274999924 & $-3,937560000038$ & & \\
\hline
\end{tabular}


Table S12: Overview of the obtained thermochemical data given in $\mathrm{kcal} / \mathrm{mol}$ for the ringclosure of the hemiaminal ether NHC-ligands

\begin{tabular}{|c|c|c|c|c|}
\hline ring closure hemiaminal ether & 5 ring & 6 ring & 5 ring $P C M$ & 6 ring $P C M$ \\
\hline E (B3LYP, cc-pVTZ) & 2,861314499940 & 3,438154500052 & 0,715179398959 & 2,709823776031 \\
\hline E ZPE (B3LYP, cc-pVTZ) & 0,801933000042 & 2,098569000048 & $-1,297890000002$ & 1,060883999888 \\
\hline U (B3LYP. cc-pVTZ) & 0,467742000102 & 1,311057000040 & $-1,656533999952$ & 0,387486000020 \\
\hline H (B3LYP, cc-pVTZ) & 1,060256999961 & 1,904199000111 & $-1,064018999951$ & 0,978747000024 \\
\hline G (B3LYP, cc-pVTZ) & $-9,567393000009$ & $-8,012433000086$ & 11,716749000000 & $-9,776810999908$ \\
\hline E (M06, cc-pVTZ) & 4,893484200062 & 3,574526999885 & 2,663005686029 & 2,429682057034 \\
\hline E ZPE (M06, cc-pVTZ) & 2,880437999998 & 1,961256000038 & 0,425106000067 & 0,909150000128 \\
\hline U (M06. cc-pVTZ) & 2,622741000007 & 1,375010999879 & 0,295317000181 & 0,282776999928 \\
\hline H (M06, cc-pVTZ) & 3,215256000008 & 1,966899000095 & 0,887204999970 & 0,874665000001 \\
\hline G (M06, cc-pVTZ) & $-7,587327000019$ & $-9,245741999895$ & 10,536108000058 & $-9,985602000022$ \\
\hline ring closure hemiaminal ether & 5 ring SMD & 6 ring SMD & & \\
\hline E (B3LYP, cc-pVTZ) & 1,145441846954 & 3,291103563094 & & \\
\hline E ZPE (B3LYP, cc-pVTZ) & $-1,011350999941$ & 1,756227000057 & & \\
\hline U (B3LYP. cc-pVTZ) & $-1,311057000040$ & 0,994421999913 & & \\
\hline H (B3LYP, cc-pVTZ) & $-0,719169000109$ & 1,586309999987 & & \\
\hline G (B3LYP, cc-pVTZ) & 11,864093999984 & $-8,571716999957$ & & \\
\hline E (M06, cc-pVTZ) & 2,8186684688011 & 3,186509303929 & & \\
\hline E ZPE (M06, cc-pVTZ) & 0,606936000036 & 1,209483000011 & & \\
\hline U (M06. cc-pVTZ) & 0,467741999960 & 0,695969999954 & & \\
\hline H (M06, cc-pVTZ) & 1,060257000103 & 1,288485000097 & & \\
\hline G (M06, cc-pVTZ) & 10,319792999963 & 10,031372999978 & & \\
\hline
\end{tabular}


Table S13: Overview of the thermochemical data given in $\mathrm{kcal} / \mathrm{mol}$ for the alcohol elimation from the hemiaminal ether NHC ligands

\begin{tabular}{|c|c|c|c|c|}
\hline alcohol loss & 5 ring & 6 ring & 5 ring $P C M$ & 6 ring $P C M$ \\
\hline E (B3LYP, cc-pVTZ) & 2,948091300063 & 18,207139499901 & 1,340373639080 & 16,975428096020 \\
\hline E ZPE (B3LYP, cc-pVTZ) & 0,242649000029 & 14,843598000018 & $-1,447743000123$ & 13,672362000124 \\
\hline U (B3LYP. cc-pVTZ) & 0,371810999988 & 14,987808000081 & $-1,868459999987$ & 13,780205999994 \\
\hline H (B3LYP, cc-pVTZ) & 0,963071999992 & 15,579696000013 & $-1,277198999983$ & 14,372094000068 \\
\hline G (B3LYP, cc-pVTZ) & 12,242174999956 & 1,862190000003 & $12,472910999944^{-}$ & 1,245848999920 \\
\hline E (M06, cc-pVTZ) & 6,181530299954 & 22,353302400047 & 4,559737116132 & 21,156849086957 \\
\hline E ZPE (M06, cc-pVTZ) & 3,437214000018 & 19,194350999939 & 1,846514999971 & 17,901476999924 \\
\hline U (M06. cc-pVTZ) & 3,669204000003 & 19,321631999974 & 1,522983000075 & 18,053211000112 \\
\hline H (M06, cc-pVTZ) & 4,260465000007 & 19,913520000048 & 2,114871000007 & 18,645098999900 \\
\hline G (M06, cc-pVTZ) & $-9,179906999990$ & 6,774108000005 & $-9,739191000003$ & 5,439852000094 \\
\hline$\underline{\text { alcohol loss }}$ & 5 ring SMD & 6 ring SMD & & \\
\hline E (B3LYP, cc-pVTZ) & 0,676132347034 & 16,027210353043 & & \\
\hline E ZPE (B3LYP, cc-pVTZ) & $-2,180705999912$ & 12,700512000011 & & \\
\hline U (B3LYP. cc-pVTZ) & $-2,046527999965$ & 12,790172999856 & & \\
\hline H (B3LYP, cc-pVTZ) & $-1,454639999891$ & 13,382688000000 & & \\
\hline G (B3LYP, cc-pVTZ) & 14,099976000042 & 0,388739999874 & & \\
\hline E (M06, cc-pVTZ) & 4,015843457882 & 20,264835623944 & & \\
\hline E ZPE (M06, cc-pVTZ) & 1,595087999965 & 14,843598000018 & & \\
\hline U (M06. cc-pVTZ) & 1,608882000072 & 14,987808000081 & & \\
\hline H (M06, cc-pVTZ) & 2,202024000000 & 15,579696000013 & & \\
\hline G (M06, cc-pVTZ) & $10,068993000026^{-}$ & 1,862190000003 & & \\
\hline
\end{tabular}




\section{Comments on the X-Ray data}

1) 6f: The 15-ring is disordered over two different conformations with $66 \%$ and $34 \%$ contribution. These could be separated and modeled sucessfully using geometric restraints. As a reasonable assumption we thereby restrained all C-C distances and $\mathrm{C}-\mathrm{C}-\mathrm{C}$ angles in the ring to be the same with a standard deviation of $0.02 \AA$. Furthermore all displacement parameters were subjected to rigid bond restraints and similarity restraints.

2) 16c: The $\mathrm{CH}-\mathrm{OH}$ unit in the backbone is disordered over two configurations with $56 \%$ and $44 \%$ contribution, which influences also the conformation of the rest of the backbone. Both situations were modeled superimposed using geometric restraints reflecting the local symmetry.

3) 18: Both ethyl arms at the aromatic ring are disordered over two conformations with $72 \%$ and $28 \%$ for $\mathrm{C} 371$ as well as $63 \%$ and $37 \%$ for C391. In each case both conformations were modeled superimposed using geometric restraints reflecting the local symmetry.

All geometric restraints were imposed using the SHELX SAME command with the default standard deviations of $0.02 \AA$. The rigid bond restraints were applied using the SHELX RIGU command with the default standard deviations 0.004 , the similarity restraints using the SHELX SIMU command, also with the default standard deviations.

\section{References}

[1] M. J. Spallek, D. Riedel, F. Rominger, A. S. K. Hashmi, O. Trapp, Organometallics 2012, 31, 1127-1132.

[2] Prepared in analogy to a) R. H. Higgins, Q. L. Eaton, L. Worth, M. V. Peterson, J. Heterocycl. Chem. 1987, 24, 255-259; b) R. H. Higgins, M. R. Watson, W. J. Faircloth, Q. L. Eaton, H. Jenkins, J. Heterocycl. Chem. 1988, 25, 383-387.

[3] All used isocyano gold(I) chlorides were synthesised analogously to a previously reported procedure (A. S. K. Hashmi, C. Lothschütz, C. Böhling, T. Hengst, C. Hubbert, F. Rominger, Adv. Synth. Catal. 2010, 352, 3001 - 3012) starting from tht $\mathrm{AuCl}$ and the respective free isocyanide.

[4] The needed acetal functionalised amines were synthesised in analogy to previously reported procedures (D. Riedel, T. Wurm, K. Graf, M. Rudolph, F. Rominger, A. S. K. Hashmi, $A d v$. Synth. Catal. 2015, 357, 1515-1523. )

[5] Gaussian 09, Revision D.01, M. J. Frisch, G. W. Trucks, H. B. Schlegel, G. E. Scuseria, M. A. Robb, J. R. Cheeseman, G. Scalmani, V. Barone, B. Mennucci, G. A. Petersson, H. Nakatsuji, M. Caricato, X. Li, H. P. Hratchian, A. F. Izmaylov, J. Bloino, G. Zheng, J. L. Sonnenberg, M. Hada, M. Ehara, K. Toyota, R. Fukuda, J. Hasegawa, M. Ishida, T. Nakajima, Y. Honda, O. Kitao, H. Nakai, T. Vreven, J. A. Montgomery, Jr., J. E. Peralta, F. Ogliaro, M. Bearpark, J. J. Heyd, E. Brothers, K. N. Kudin, V. N. Staroverov, T. Keith, R. Kobayashi, J. Normand, K. Raghavachari, A. Rendell, J. C. Burant, S. S. Iyengar, J. Tomasi, M. Cossi, N. Rega, J. M. Millam, M. Klene, J. E. Knox, J. B. Cross, V. Bakken, C. Adamo, J. Jaramillo, R. Gomperts, R. E. Stratmann, O. Yazyev, A. J. Austin, R. Cammi, C. Pomelli, J. W. Ochterski, R. L. Martin, K. Morokuma, V. G. Zakrzewski, G. A. Voth, P. Salvador, J. J. Dannenberg, S. Dapprich, A. D. Daniels, O. Farkas, J. B. Foresman, J. V. Ortiz, J. Cioslowski, and D. J. Fox, Gaussian, Inc., Wallingford CT, 2013. 
[6] a) P. Hohenberg, W. Kohn, Phys. Rev., 1964, 136, B864-B71; b) W. Kohn and L. J. Sham, Phys. Rev., 1965, 140, A1133-A38; c) R.G. Parr and W. Yang, Density-functional Theory of atoms and molecules, Oxford Univ. Press, 1989; d) W. Koch, Max C. Holthausen, A Chemist's Guide to Densitiy Functional Theory, Wiley, 2001.

[7] a) A. D. Becke, Phys. Rev. A, 1988, 38, 3098-3100; b) A. D. Becke, J. Chem. Phys., 1993, 98, 5648-5652; c) C. Lee, W. Yang, R. G. Parr, Phys. Rev. B, 1988, 37, 785-789; d) S. H. Vosko, L. Wilk, M. Nusair Can. J. Phys., 1980, 58, 1200-1211.

[8] Y. Zhao, D. G. Truhlar, Theor. Chem. Acc., 2008, 120, 215-241.

[9] S. Grimme, J. Antony, S. Ehrlich, H. Krieg, J. Chem. Phys., 2010, 132, 154104.

[10] a) T. H. Dunning Jr., J. Chem. Phys., 1989, 90, 1007-1023; b) R. A. Kendall, T. H. Dunning Jr., and R. J. Harrison, J. Chem. Phys., 1992, 96, 6796-6806; c) D.E. Woon and T. H. Dunning Jr., J. Chem. Phys., 1993, 98 1358-1371; d) K. A. Peterson, D.E. Woon, and T. H. Dunning Jr., J. Chem. Phys., 1994, 100, 7410-7415; e) A. K. Wilson, T. van Mourik, and T. H. Dunning Jr., J. Mol. Struct. (Theochem), 1996, 388, 339-349.

[11] a) K.A. Peterson, D. Figgen, E. Goll, M.Dolg, H. Stoll, J. Chem. Phys., 2003, 119, $11113-$ 11123; b) K.A. Peterson, C. Puzzarini, Theor. Chem. Acc., 2005, 114, 283-296.

[12] For detailed information on the PCM solvent model please see the following review article and the references cited therein: J. Tomasi, B. Mennucci, and R. Cammi Chem. Rev., 105 (2005) 2999-3093.

[13] A. V. Marenich, C. J. Cramer, and D. G. Truhlar, J. Phys. Chem. B, 113 (2009) 6378-6396 